\title{
A Feasibility Study of a Group-Format, Ecologically Oriented Neurorehabilitation of Memory (EON-Mem) Program in Older Adults
}

Kayla Michelle Frodsham

Brigham Young University

Follow this and additional works at: https://scholarsarchive.byu.edu/etd

Part of the Family, Life Course, and Society Commons

\section{BYU ScholarsArchive Citation}

Frodsham, Kayla Michelle, "A Feasibility Study of a Group-Format, Ecologically Oriented Neurorehabilitation of Memory (EON-Mem) Program in Older Adults" (2021). Theses and Dissertations. 9089.

https://scholarsarchive.byu.edu/etd/9089

This Dissertation is brought to you for free and open access by BYU ScholarsArchive. It has been accepted for inclusion in Theses and Dissertations by an authorized administrator of BYU ScholarsArchive. For more information, please contact ellen_amatangelo@byu.edu. 
A Feasibility Study of a Group-Format, Ecologically Oriented Neurorehabilitation of Memory (EON-Mem) Program in Older Adults

\author{
Kayla Michelle Frodsham
}

\begin{abstract}
A dissertation submitted to the faculty of
Brigham Young University

in partial fulfillment of the requirements for the degree of

Doctor of Philosophy
\end{abstract}

\author{
Michael J. Larson, Chair \\ Scott Baldwin \\ Gary Burlingame \\ Derin Cobia \\ Ramona O. Hopkins
}

Department of Clinical Psychology

Brigham Young University

Copyright (C) 2021 Kayla Michelle Frodsham

All Rights Reserved 


\begin{abstract}
A Feasibility Study of a Group-Format, Ecologically Oriented Neurorehabilitation of Memory
\end{abstract} (EON-Mem) Program in Older Adults

\author{
Kayla Michelle Frodsham \\ Department of Psychology, BYU \\ Doctor of Philosophy
}

Older adults often show declines in subjective and objective memory performance relative to younger adults. One potential path for helping older adults with memory may be compensatory memory training programs. Compensatory memory training programs teach strategies to manage memory impairment. Traditional compensatory memory training programs tend to be highly specific to a task and often do not generalize to other memory tasks. Ecologically Oriented Neurorehabilitation of Memory (EON-Mem) is a method for teaching memory strategies that may generalize for efficient use in everyday contexts. We performed a feasibility study to determine the value of pursuing a group-based version of EON-Mem with older adults in a future larger-scale randomized controlled trial. The current feasibility study took place in two phases with two separate samples. The first sample consisted of five separate groups of healthy young adults $(n=39)$. The second sample consisted of three separate groups of older adults $(n=26)$. We collected data on recruitment, treatment adherence, memory improvement, drop-out rate, cost, time spent, and participant-report data on barriers to successful implementation of EON-Mem treatment. We also collected data on memory performance and overall cognitive functioning. In order to assess improvement before and after treatment within our sample, reliable change indices were calculated using the Repeatable Battery for the Assessment of Neuropsychological Status (RBANS) delayed memory index score. Participants first performed a baseline assessment (traditional and ecological memory tasks, general cognition tasks, emotional functioning, demographics). After the baseline assessment, participants attended one treatment session (90 minutes) per week for a total of seven group-based EON-Mem treatment sessions. Thirty-nine young adults and 26 older adults enrolled in the study; 20 young adults and 10 older adults completed the treatment sessions. We prematurely ended older adult group treatment sessions due to the COVID-19 pandemic. All participants performed a post-intervention assessment using alternate versions when available. Attendance rates were low for the young adult sample (51\% completed the treatment) and as expected for the older adult sample when accounting for COVID-related changes (77\% eligible for completer status prior to cancelling sessions). Twenty percent of each sample reliably improved on the RBANS delayed memory index score before and after treatment. Costs were higher than expected $(\$ 345$ and 18.6 research hours for each young adult participant; $\$ 319$ and 16.9 research hours for each older adult participant). Subjectively, both samples reported enjoying the interactions with others and the presentation of the treatment, but disliked peg words. Overall, although a randomized controlled trial of groupformat EON-Mem in older adults is feasible, such a study may or may not be cost-effective depending on the resources and goals of the researcher.

Keywords: feasibility, older adults, memory training, compensatory 


\section{ACKNOWLEDGEMENTS}

I feel confident many doctoral students wish for the ability to grant $\mathrm{PhDs}$ to others in their lives who have supported their dissertation. I am no exception. If I could, I would give my husband, Scott Frodsham, a PhD in whatever he wants for the kindness, understanding, excitement, belief, and empathy he has offered me throughout my dissertation experience. So many other family members, especially my mom and my sister-in-law, have watched our boys, ignited faith, and cheered us on. Dedication to the project was apparent in the hours, attitude, and initiative of my "army of research assistants." Hilary Smith gave to the project through preparing materials, contributing to the method for the project, and adapting the materials to group format. Finally, Michael Larson's commitment to student development, and to my development specifically, was obvious through the frequent revisions, constant encouragement, high beliefs, and sincere support. He is a wonderful mentor. Maybe a completion of a dissertation is possible for a single person. But the gratitude and love in my heart for all involved suggests otherwise. Thank you, truly. 


\section{TABLE OF CONTENTS}

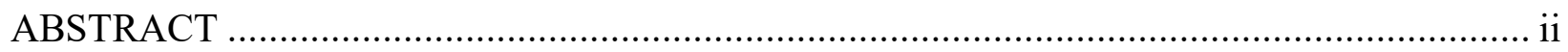

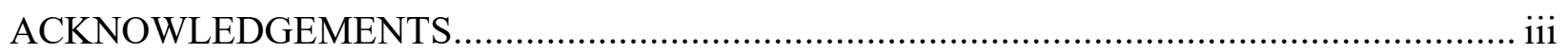

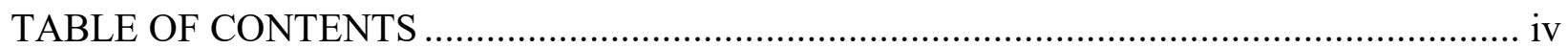

LIST OF FIGURES ....................................................................................... viii

A Feasibility Study of a Group-Format, Ecologically Oriented Neurorehabilitation of Memory

(EON-Mem) Program in Older Adults...................................................................

Evidence for Remediation Training .......................................................................2

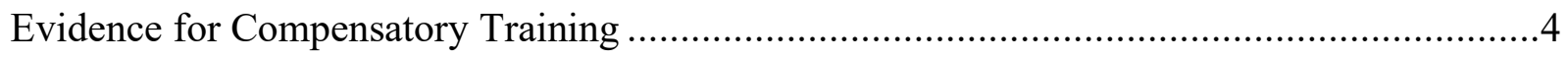

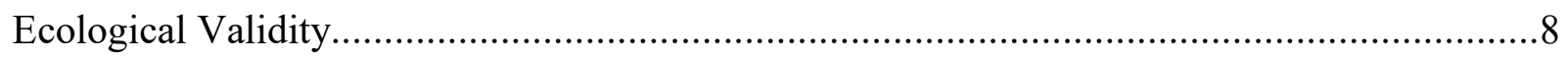

Ecologically Oriented Neurorehabilitation of Memory …............................................. 10

Eon-Mem Modules................................................................................................. 10

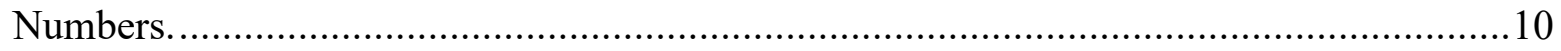

Object location. ........................................................................................... 11

Oral and written information......................................................................... 11

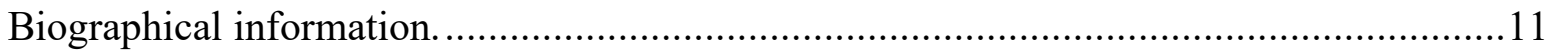

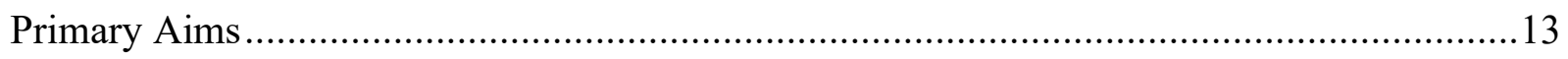

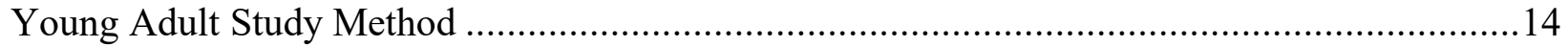

Young Adult: Participants ...................................................................................... 14

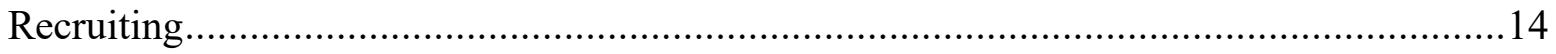

Exclusion criteria....................................................................................... 16

Young Adult: Procedures ........................................................................................ 17

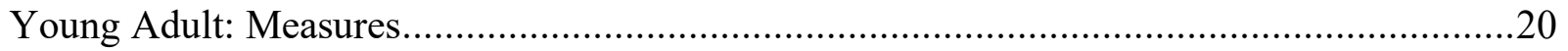


Intelligence Quotient (IQ) estimate ......................................................................2 21

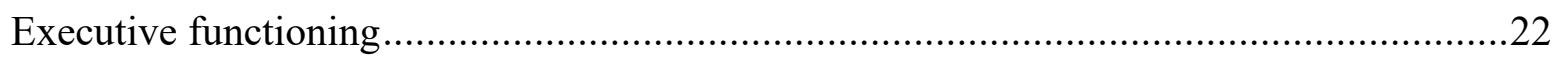

Depression and anxiety symptoms ......................................................................2 23

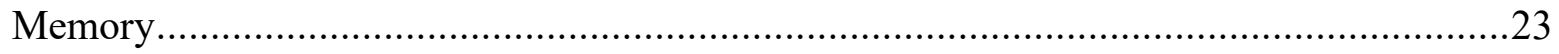

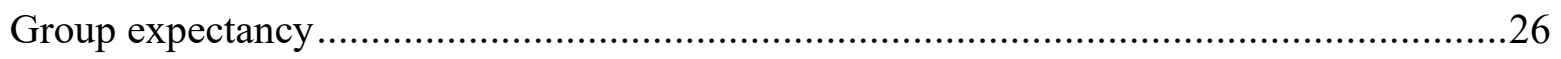

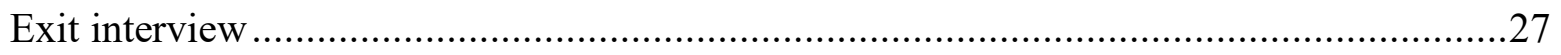

Young Adult: Weekly Measures.........................................................................28

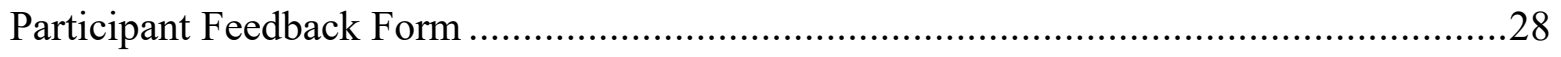

Group Climate Questionnaire, short form Engagement subscale ...............................28

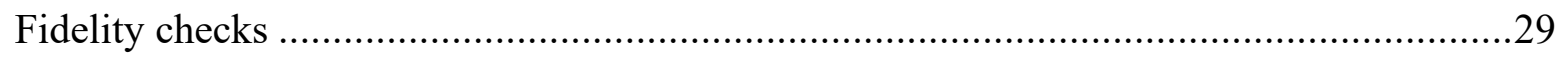

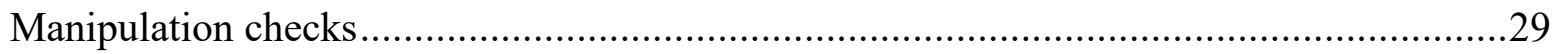

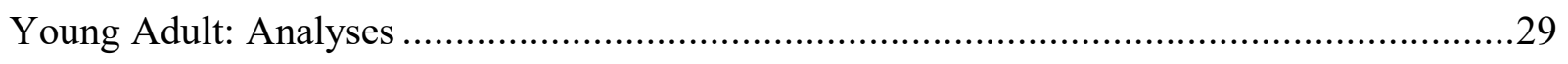

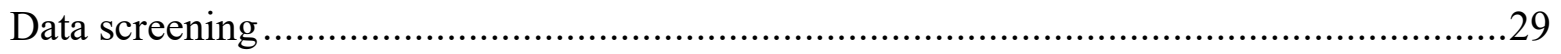

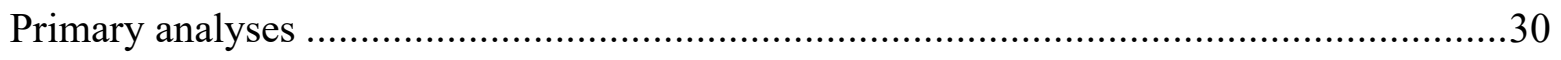

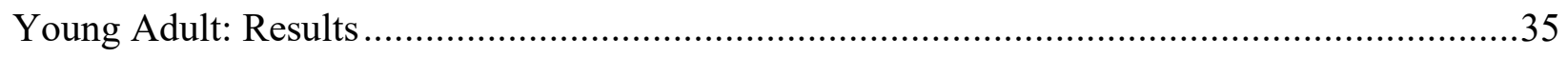

Aim 1: To Provide Descriptive Statistics about Adherence to Treatment, Cognitive and Emotional Functioning, Participant Feedback, and Relevant Demographic Information ........35

Aim 2: To provide Information Regarding Resources Required for EON-Mem ...................51

Aim 3: To Assess Change in Memory Performance........................................................54

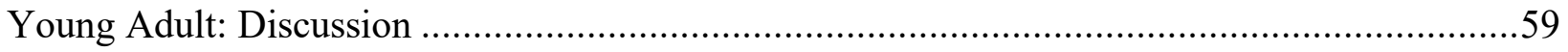

Revisions for Older Adult Sample made in Response to Young Adult Findings ..................61

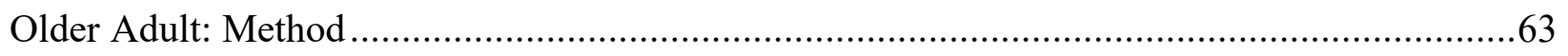

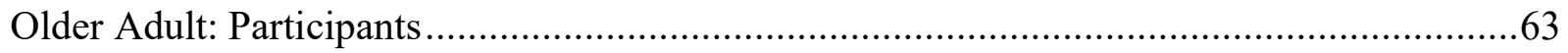




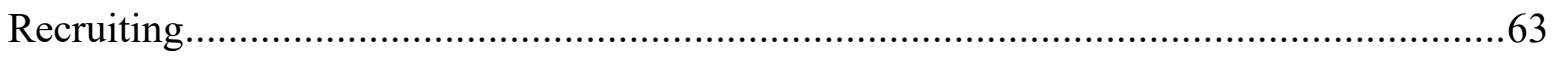

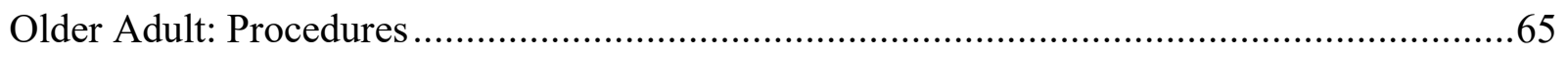

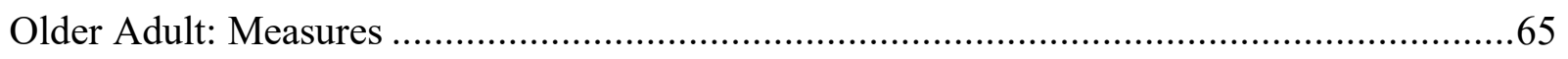

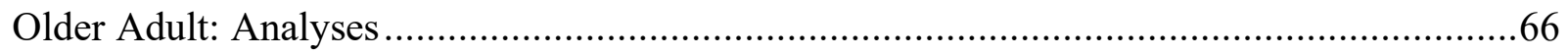

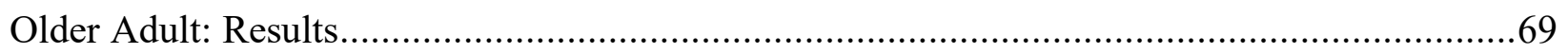

Aim 1: To Provide Descriptive Statistics about Adherence to Treatment, Cognitive and Emotional Functioning, Participant Feedback, and Relevant Demographic Information .......69

Aim 2: To provide Information Regarding Resources Required for EON-Mem ...................86

Aim 3: To Assess Change in Memory Performance.................................................. 90

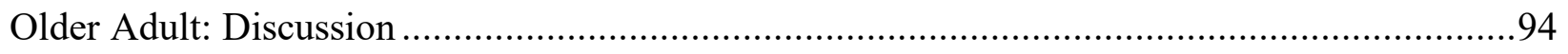

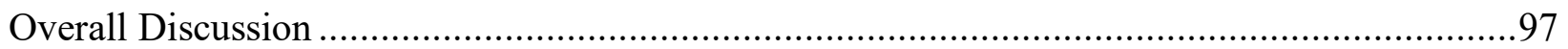

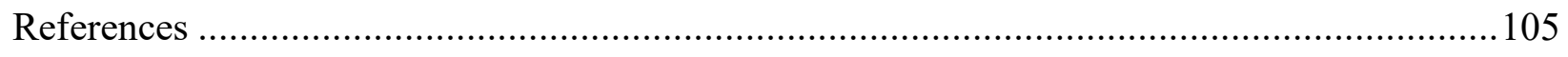

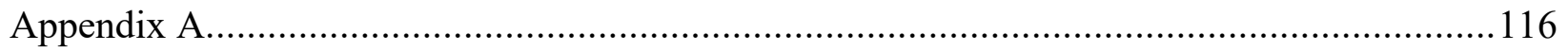

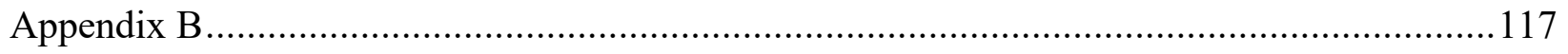

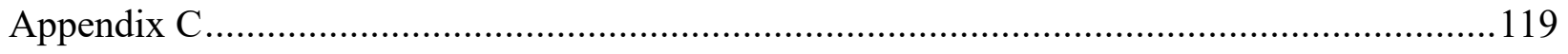




\section{LIST OF TABLES}

Table 1: Treatment Session Content .........................................................................20

Table 2: Young Adult: Reliabilities for Calculating Reliable Change Indices ..........................34

Table 3: Young Adult: Description of Attendance and Homework for the Sample...................36

Table 4: Young Adult: Quantitative Participant Feedback from Weekly Treatment Session

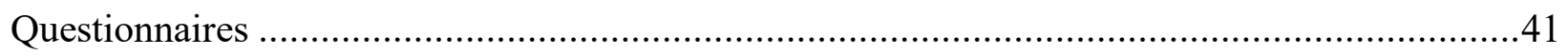

Table 5: Young Adult: Reported Facilitators and Barriers to EON-Mem Treatment ................42

Table 6: Young Adult: Baseline Information for Completers in the Young Adult Sample.........48

Table 7: Young Adult: Baseline Information for Improvers in the Young Adult Sample...........49

Table 8: Young Adult: Baseline Information for Homework Completion Groups in the Young

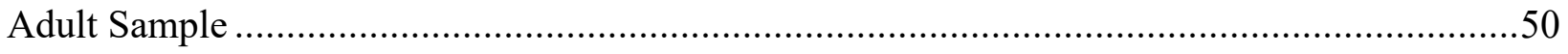

Table 9: Young Adult: Cost and Time Required for Implementation ..................................52

Table 10: Older Adult: Reliabilities for Calculating Reliable Change Indices ........................67

Table 11: Deviations from Pre-registration/Prospectus ............................................6 68

Table 12: Older Adult: Description of Attendance and Homework for the Sample ..................70

Table 13: Older Adult: Quantitative Participant Feedback from Weekly Treatment Session

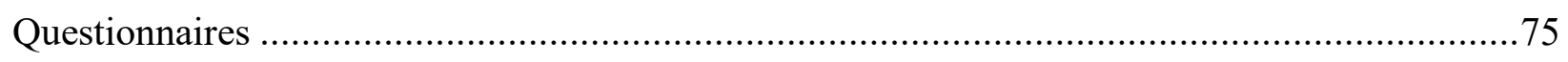

Table 14: Older Adult: Reported Facilitators and Barriers to EON-Mem Treatment .................76

Table 15: Older Adult: Baseline Information for Completers in the Older Adult Sample ..........83

Table 16: Older Adult: Baseline Information for Improvers in the Older Adult Sample ............84

Table 17: Older Adult: Baseline Information for Homework Completion Groups in the Older

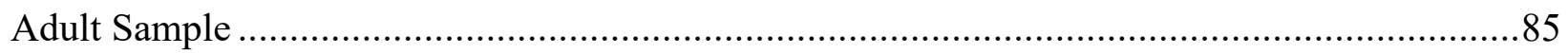

Table 18: Older Adult: Cost and Time Required for Implementation...................................87 


\section{LIST OF FIGURES}

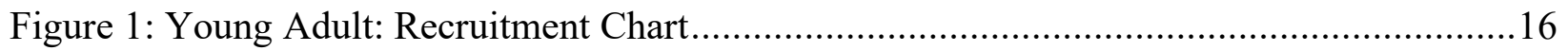

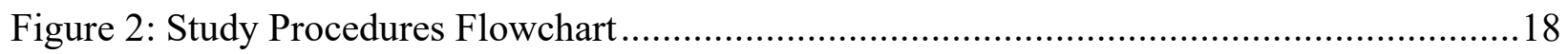

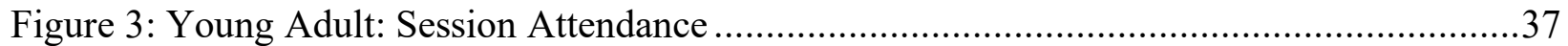

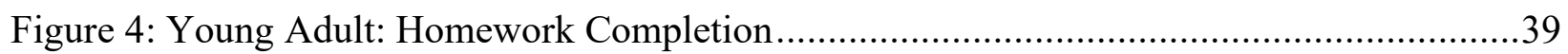

Figure 5: Young Adult: GCQ-S Engagement Subscale ......................................................4

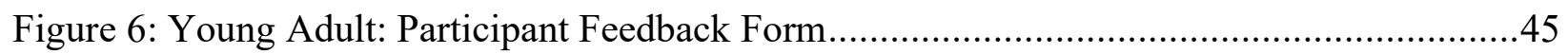

Figure 7: Young Adult: Average Session Length...................................................................5

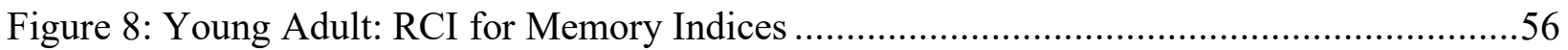

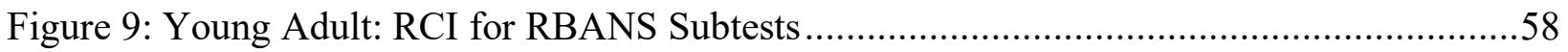

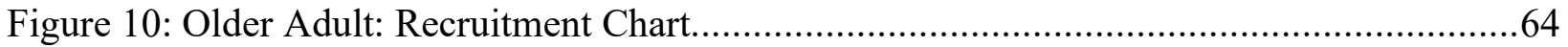

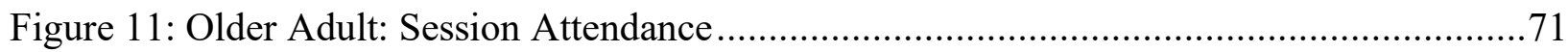

Figure 12: Older Adult: Homework Completion..................................................................

Figure 13: Older Adult: GCQ-S Engagement Subscale...........................................................

Figure 14: Older Adult: Participant Feedback Form ……...................................................

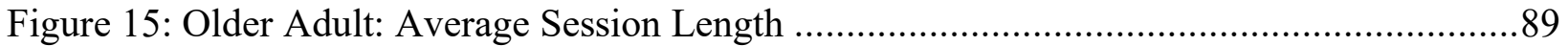

Figure 16: Older Adult: RCI for Memory Indices...............................................................91

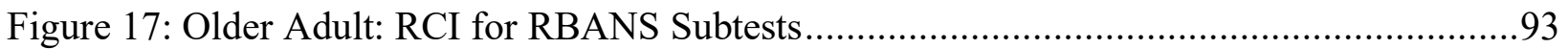




\section{A Feasibility Study of a Group-Format, Ecologically Oriented Neurorehabilitation of Memory (EON-Mem) Program in Older Adults}

The prevalence of memory complaints in community samples of older adults over age 65 ranges from $25 \%$ to $50 \%$ (Jonker et al., 2000). For example, in a sample of 499 older adults aged 65 years or older, $27 \%$ reported that they had some problems with their memory (Fritsch et al., 2014). Thirty-two percent of 1,637 participants 65 years and older, mean age $=74.7(S D=6.9)$, reported memory complaints in another large population study (Montejo et al., 2011). Selfreported memory complaints are negatively associated with quality of life and independence in daily living skills (Montejo et al., 2012). In addition to self-reported "subjective" ratings of poor memory, older adults consistently show decreased objective memory performance on neuropsychological and experimental measures of learning and recall compared to younger adults (Old \& Naveh-Benjamin, 2008). For example, healthy older adults consistently perform worse than younger adults on measures of episodic memory (i.e., memory of events), both in laboratory tests and tests representing everyday memory by approximately a standard deviation difference (Prull et al., 2000). In a meta-analysis of 3,192 older adults (mean age $=71$ years) and 3,197 younger adults (mean age $=21$ years), older individuals performed worse than younger individuals with a large effect size (Cohen's $d=.84$; Old \& Naveh-Benjamin, 2008). Given the prevalence of memory concerns and impairments associated with aging, effective methods to treat memory difficulties is important to potentially slow age-related memory decline, improve quality of life, and improve independence in daily living skills (Montejo et al., 2012).

One possible avenue for improving memory performance and decreasing self-reported memory concerns in older adults is memory training programs (Hertzog et al., 2008). Memory training programs are typically grouped into two types: remediation/restorative training and 
compensatory/strategy training (Klingberg, 2010). Remediation training theories argue that a focus on repeated practice of the direct task will strengthen and develop new synaptic connections thereby improving memory performance. The repeated practice is specific to a cognitive domain and increases in difficulty over time in the hopes of strengthening the impaired skill, such as practicing rote memorization of face-name pairs to improve memory for names (Barman et al., 2016). The remediation training approach is sometimes referred to as a "mental muscle approach" (Klingberg, 2010).

Compensatory/strategy training, on the other hand, is focused on teaching strategies to manage memory difficulties (Klingberg, 2010). The goal of compensatory training is not to change the impaired function directly, but to use resources to work around (i.e., compensate for) the impaired memory function (Barman et al., 2016; Rogers et al., 2000). Compensatory training helps individuals change their approach to a task, such as using a calendar to compensate for impaired memory of events or using internal strategies to help a person more efficiently remember a list or object locations (Barman et al., 2016; Rogers et al., 2000).

\section{Evidence for Remediation Training}

In a study called the Improvement in Memory with Plasticity-based Adaptive Cognitive Training (IMPACT), Smith et al. (2009) found participants receiving remediation training obtained a higher composite memory score than controls (effect size $d=.3$ ) in a sample of 487 older adults (65 and older). The remediation training consisted of 40 one-hour computerized sessions in which participants completed exercises of increasing difficulty aimed at improving auditory processing (e.g., discriminating, matching, or recognizing confusable syllables).

Mahncke et al. (2006) also used a brain plasticity-based training program in a sample of 182 participants aged 60 or older and found support for remediation memory training. 
Specifically, Mahncke et al. (2006) found the group that received memory training had improved memory (e.g., identifying and sequencing vocalizations, discriminating and matching syllables, reconstructing short words and instructions, answering questions about short stories), whereas the active group (watched education lectures on computer) and no-contact control groups did not show significant gains in memory performance. The experimental group also had significant improvement on a standardized measure of auditory memory (Repeatable Battery for the Assessment of Neuropsychological Status (RBANS); $p=.02 ; d=.3$ ) compared to the control group.

Hawley and Cherry (2008) used spaced retrieval remediation training to improve memory for faces and names. In spaced retrieval face-name association tasks, participants are told the name of a person in a photograph, and then after a delay asked to retrieve a photograph when given a name (Hawley \& Cherry, 2008). If correct, the experimenter increases the time between teaching the face-name pair and asking the participant to recall the picture associated with the face; if the response is incorrect, the experimenter gives the correct answer and decreases the time between teaching and recall (Hawley \& Cherry, 2008). The practice was repeated several times over 12 sessions (Hawley \& Cherry, 2008). In a small study of 12 adults with Alzheimer's Disease, participants assigned to a spaced retrieval task performed better on face-name association tasks (recalling a name-face pair when prompted with a picture of a person) and better on transfer to live person tasks (recognizing and remembering a live person's name when taught the face-name pair with a photograph) compared to healthy control participants (Hawley \& Cherry, 2008).

One study did not support effects of remediation training on memory performance. Oh et al. (2018) utilized "Smartphone-based brain Anti-aging and Memory Reinforcement Training” 
(SMART), a remediation task composed of several tasks with increasing difficulty in domains of attention, working memory, and memory. Fifty-three participants (ages ranging from 50-68) engaged in the tasks for 15-20 minutes per day, five days per week, across 8 weeks (Oh et al., 2018). There was no significant difference in memory performance across time or between groups (control groups included a waitlist control and another remediation training, Fit Brains; Oh et al., 2018). Overall, however, remediation training based on repeated practice has some support for improving memory with small-to-medium effect sizes in older adults and in older adults with suspected Alzheimer's Disease.

\section{Evidence for Compensatory Training}

There is also compelling evidence for the utility of compensatory strategy memory training in older adults. In a large meta-analysis of 3,797 participants, mean age $=73$ years $(S D=$ 4.3), Gross et al. (2012) tested the effect of memory strategy training on memory outcomes including nine different mnemonic strategies and external memory aids from 33 different studies. Overall, participants who received memory strategy training improved from pre-intervention to post-intervention on memory tasks with an average effect size of 0.4 , which was significantly better than performance of control groups (0.06; Gross et al., 2012). In another meta-analysis of 1,539 older adults, mean age $=69.1$, individuals who received mnemonic strategy training improved from pre-intervention to post-intervention on memory tasks with an average effect size of .73, which was significantly better than performance of control (.38) and placebo groups (.37; Verhaeghen et al., 1992).

Similarly, in a sample of 2,832 older adults, mean age $=73.6$ years, known as the Advanced Cognitive Training for Independent and Vital Elderly (ACTIVE) trial, Rebok et al. (2014) reported compensatory strategy training was associated with improvements in memory, 
reasoning, and processing speed when tested immediately after training and again 5-years postbaseline. There was no effect of memory training on memory performance at the 10 year followup (Rebok et al., 2014). In a subsample of 629 older adults, mean age $=73.5(S D=6.0)$, from the ACTIVE trial, Rebok et al. (2013) found for those who completed at least eight of the initial sessions, memory improved from initial assessment to post-treatment. Although all groups (adherent and non-adherent) declined in memory score compared to their own initial score over five years, individuals in the adherent group had better memory scores at 5-years post-treatment than the non-adherent groups (Rebok et al., 2013).

Several, small-scale studies demonstrate mixed evidence of compensatory training for memory difficulties. In a study examining a sample of 53 older adult participants, mean age $=$ $72.4(S D=9.1)$, the experimental group was taught internal and external memory strategies, as well as information regarding the effects of normal aging, nutrition, and mood on memory (Fairchild \& Scogin, 2010). The experimental group performed better than controls on immediate memory (i.e., memory tested within a few minutes after teaching) and delayed memory (i.e., memory tested 20-30 minutes after teaching) for face-name pairs (Fairchild \& Scogin, 2010). In a sample of 29 older adult (ages 60+), Chan et al. (2018) found implementation of a memory intervention involving psychoeducation, mnemonics, and at-home assignments was associated with significant improvement in verbal and visual memory scores at post-test and 18month follow-up (SD between .6 and 1.4). There were no significant differences on memory performance measures between the memory intervention and the active control group (Deijan Mind-body Intervention; Chan et al., 2018). However, in a sample of 265 older adults, McDougall et al. (2010) found the experimental group (employing memory strategies, stressreduction strategies, and education regarding memory and aging) did not show gains on any 
memory measures. Taken together, a number of studies suggest compensatory training is beneficial for memory improvement in older adults (small-to-medium effect size), alternatively several studies also find no significant effect of compensatory training.

Although both remediation and compensatory training have evidence supporting their use in older adult participants, some research supports the primary use of compensatory training over remediation training. For example, one memory training specifically utilizing compensatory strategies was associated with greater pre/post-improvement in personal memory goals compared to a memory training utilizing repetitive practice of memory/attention tasks (Frankenmolen et al., 2018). Moreover, internal strategy use was associated both with improvement on personal memory goals and with decline in subjective memory complaints (Frankenmolen et al., 2018). Additionally, a meta-analysis by Hudes et al. (2019) found memory strategy training was associated with positive outcomes in self-efficacy for memory, memory strategy use, and participant-reported memory ability. Authors have pointed out problems with remediation training as a memory training program. One major problem with remediation theory is the approach incorrectly assumes memory is a unitary process, further assuming specific memory practice will help all memory problems (Ptak et al., 2010).

In actuality, forming memories is complex and involves several neural systems (e.g., sensory modalities, medial temporal lobe, frontal regions, basal ganglia) and several mechanisms (e.g., short-term/working memory, higher order processes for forming associations between stimuli, encoding long-term memories, storage of long-term memories, retrieval of stored information; Ericsson, 1985). Moreover, the different neural systems listed above involve several areas of the brain including the hippocampus, association areas such as the prefrontal cortex, perirhinal, piriform, entorhinal, and parahippocampal cortices, amygdala, and neocortical areas 
(Eichenbaum, 2000; Rattenborg \& Martinez-Gonzalez, 2011). Ptak et al. (2010) also states many remediation training methods do not generalize to everyday tasks or to untrained material. That is, cognitive remediation programs improve performance on trained tasks, but have limited efficacy in transferring to everyday cognitive performance; suggesting compensatory training may be better for day-to-day application (Simons et al., 2016). Despite the aforementioned benefits of compensatory training and drawbacks of remediation training, current compensatory memory training practices demonstrate two significant weaknesses addressed in the following sections: affordability/accessibility and ecological validity.

\section{Affordability and Accessibility of Memory Training}

Two barriers often faced in obtaining memory training are affordability and accessibility. Individuals with memory deficits often do not receive memory rehabilitation, and if they do, memory rehabilitation is often brief (das Nair et al., 2019). The specialization required of clinicians to perform memory training and the time required to participate in the intervention is associated with high treatment costs, often not fully covered by insurance. Further, waiting lists are often months long, even for those willing to pay for treatment. Perhaps one contributing factor to high costs and long waiting lists is the typical format of one-on-one therapist-to-client trainings.

Group format may offer increased affordability and accessibility over one-on-one treatment designs. In psychotherapy research broadly, the majority of studies suggest individual format and group format are equivalent in outcomes measured, with economic advantage for group format (Burlingame et al., 2013). Important factors in administering group treatment include group climate and cohesion, a "positive bond between the member and group" (Burlingame et al., 2013, p. 670). Cohesion predicts outcome across a variety of group 
psychotherapy outcome studies with a medium effect size $(r=.3$; Burlingame et al., 2013). In memory trainings specifically, Verhaeghen et al. (1992) found participants performed better on memory tasks when memory strategy training was taught in groups compared to when it was taught individually $\left(R^{2}=.4, p=.001\right)$. Memory training in groups may have advantages relative to individually administered training: normalization of fear and anxiety, increased self-efficacy, development of social support networks, and a higher likelihood individuals will use the skills learned (Thickpenny-Davis \& Barker-Collo, 2007; Wilson, 1995; Wilson \& Moffatt, 1992). Further, working collaboratively on a common problem is associated with maintenance of strategy use on collaborative tasks (Rebok et al., 2007). Thus, group format may be a next step in providing memory training treatment due to potential benefits such as social interaction and affordability.

\section{Ecological Validity}

Another weakness in memory training with older adults is poor ecological validity. For example, across 33 studies for a total of 1,539 older adults, mean age $=69$ years, compensatory memory training was consistently associated with a larger effect size on target memory tasks than on non-target memory tasks (effect sizes could not be directly compared as they shared the same sample; Verhaeghen et al., 1992). A target memory task is a task to which a trained memory strategy (e.g., method of loci in which one imagines objects from a list along different positions in a familiar place) may be applied and is a direct test of memory training (e.g., using method of loci for a word list); a non-target memory task is a task in which the trained memory strategy cannot be readily applied, and is therefore considered a test of ecological validity (e.g., method of loci and face-name pairs; Verhaeghen et al., 1992). Thus, Verhaeghen et al. (1992) suggest compensatory memory training is somewhat task-specific and lacks ecological validity. 
Rebok et al. (2007) also suggest traditional compensatory memory training tends to be highly specific to a task and does not generalize to other memory tasks: the authors cite an example in which training on the method of loci strategy impacted performance on word recall, but not on number recall. Taken together, despite being a better alternative to remediation training, some research suggests compensatory memory training has poor ecological validity.

Studies to date show promise for compensatory memory training effects extending to everyday life in older adults (Bottiroli et al., 2017; Frankenmolen et al., 2018). One study by Bottiroli et al. (2017) implemented a self-guided strategy-adaptation intervention in which participants were not only taught memory strategies but how to apply these strategies in a variety of situations. These authors found the experimental group compared to controls performed better on both the practiced tasks (remembering word pairs) and on non-practiced tasks (remembering face-name pairs, grocery list), suggesting that compensatory memory training may have ecological validity when participants are taught how to adapt strategies (Bottiroli et al., 2017). Rosi et al. (2018) similarly found 44 participants, mean age $=68.7(S D=6.1)$, in a memory strategy training group (e.g., verbal and visual associative techniques) demonstrated pre- to posttest improvements in both practiced and non-practiced memory tasks. In another study, between $39 \%$ to $94 \%$ of participants reported using mnemonics taught in class in their daily lives (Chan et al., 2018). Of note, the number of self-reported strategies employed was not significantly correlated to memory improvement at 18-month follow-up (Chan et al., 2018). Finally, Frankenmolen et al. (2018) found that, although both a memory strategy training group and a control memory training group improved on personal memory treatment goals, the memory strategy training group had a higher percentage of participants with clinically significant improvement at six-month follow-up compared to the control group (76\% and $23 \%$ 
respectively). Moreover, improvement in internal strategy use was related to the improvement in the memory strategy training group (Frankenmolen et al., 2018). In sum, although there is some recent evidence for ecological validity in compensatory memory training programs for older adults, there is still a great need for improved ecological validity in current compensatory memory training strategies.

\section{Ecologically Oriented Neurorehabilitation of Memory}

Ecologically Oriented Neurorehabilitation of Memory (EON-Mem) is one potential method for teaching simple memory strategies which may be used in ecologically valid everyday contexts (Stringer, 2011). EON-Mem is a manualized compensatory memory strategy protocol which teaches mnemonic strategies and simple memory techniques based on the acronym of WOPR: write, organize, picture, rehearse (Stringer, 2011). Participants and therapists first set goals together and then therapists teach participants how to apply WOPR to seven different types of information: numbers, appointments and future tasks, object location, oral information, written information, routes, and biographical information (Stringer, 2011). Participants are provided daily homework assignments from the manual allowing them to learn and apply strategies to real-world situations between sessions (Stringer, 2011). In the current study, we followed a modified schedule for therapy sessions. Namely, to improve feasibility, we shortened the number of sessions by removing modules related to route finding and appointments/future tasks. Both of these modules have intuitive solutions for remembering, such as global positioning system and calendars. The remaining information modules are described below.

\section{Eon-Mem Modules}

Numbers. Using the WOPR system, participants first write the number down. Next, participants organize numbers by "chunking" them into groups of two or three, such as chunking 
1-4-6-8 into 14 and 68. Participants then use the peg system to help them picture the numbers. The peg system is a memory aid which pairs a rhyming word with each number for numbers 0 12, for example "1-bun" and "6-sticks." The peg system not only enables easier memorization with the association of a rhyme but enables visualization of the numbers by providing a picture for each number. Finally, the participants repeat their images and associated words in the rehearsal step.Object location. Participants do not use the write step, as this is not applicable to object locations; the first step for object locations is to organize by pre-selecting good locations for each object based on where the objects will be used, where similar objects are kept, or where one will see the object before leaving. Next, participants picture the object in the decided upon location. Finally, the participants repeat the locations and pictures of locations in their mind.

Oral and written information. Using the WOPR system, participants first write anticipated questions regarding spoken or written information. The participants then write out answers to these questions as they listen to/read the information, adding and revising questions as necessary. Next, participants organize questions and corresponding answers according to topic. Participants also use acronyms (organize a set of letters that stand for each of something in a list) and acrostics (create a sentence from making words from the first letter of each word in a list) to organize written and oral information. In the picture phase, participants use peg words for dates and lists of facts. Participants also associate pictures that sound like names to pictures of related facts. Finally, in the rehearsal phase, participants use their questions to practice retrieval of information, as well as spend time recalling acronyms, acrostics, and pictures.

Biographical information. Participants first write down names, distinguishing facial features, and important facts about those they meet and want to remember. Next, they organize people by category (e.g., supervisor, potential friend) and biographical information by topic (e.g., 
training experience, work history). In the picture step, participants associate the distinguishing facial feature with the person's name in addition to picturing the person doing activities related to their important biographical facts. In rehearsal, the participant practices associating the name with the facial feature as well as recalling important facts from the mental picture they created.

To date, EON-Mem has shown promise in individuals with traumatic brain injury (TBI), stroke, and other cognitive disability For example, in a sample of 33 participants referred for cognitive training (15 TBI, 12 stroke, 6 other neurological condition), participants significantly improved on several tests of everyday memory simulations from pre-intervention to postintervention (Stringer, 2011). One other group tested EON-Mem in a sample of patients with cognitive disability via two methods: face-to-face and remote instruction (Sporner, 2013). Overall, authors found patients improved on memorization of stories, prospective memory, selfawareness of memory deficits, and strategies employed across both treatment delivery types (effect sizes not reported; Sporner, 2013). Notably, EON-Mem has only been tested in the aforementioned studies with diverse samples and has not been tested in a clinical trial, severely limiting our ability to make strong claims of the intervention's effectiveness. Moreover, EONMem has yet to be systematically implemented in older adults or in a group format.

Thus, the primary purpose of the current study was to collect the information required for determining the feasibility of a future clinical trial of EON-Mem, specifically with older adults in group-format. Considering EON-Mem had never been tested in a group or with older adults, a feasibility study was needed to determine the value of pursuing a group-based memory program in a larger-scale study. A feasibility study is a small study that helps inform the design of a future larger study, such as a randomized-controlled trial (Arnold et al., 2009; Thabane et al., 2010). For instance, feasibility studies provide information about protocol implementation (e.g., 
recruitment, eligibility, drop-out), adherence (e.g., attendance, homework completion), resources (e.g., cost of materials, time required for sessions), and assessment (e.g., standard deviation of outcome measures; Arnold et al., 2009; Leon et al., 2011; Thabane et al., 2010). Because the purpose of a feasibility study is to prepare information for a clinical trial as opposed to determine treatment effects, we did not include any non-rehabilitation control groups in our study.

Although our target population was older adults, we first performed a feasibility study in a healthy young adult sample to identify barriers of implementing EON-Mem in a group format. After completing a feasibility study in healthy young adults, we adjusted the protocol and continued the feasibility study with older adults. The primary aims (below) detail the specific information gathered and analyzed to accomplish our primary purpose of performing a feasibility study for EON-Mem in group-format with older adults.

\section{Primary Aims}

1) To provide descriptive statistics about (a) adherence to treatment (e.g., attendance, homework completion), (b) cognitive functioning (e.g., estimated intelligence, general cognition, executive functioning), (c) emotional functioning (e.g., anxiety and depressive symptoms), (d) participant feedback (e.g., Participant Feedback Form, GCQ-S Engagement subscale), and (e) relevant demographic information for those who completed EON-Mem in a group-format and for those who improved after EON-Mem in a group-format, specifically to inform future randomized controlled trials (RCT).

2) To provide information regarding resources required for EON-Mem implementation in a group-format for future RCT. To provide descriptive statistics about (a) the cost required for implementation, (b) the time required for implementation, and (c) recruitment information for the samples. 
3) To assess change in memory performance for healthy young adults and older adults from preEON-Mem training to post-EON-Mem training (separately). We expected both healthy young adults and older adults receiving EON-Mem in our sample would improve from pre-intervention to post-intervention on both everyday memory tests and traditional, standardized memory tasks (Prull et al., 2000; Verhaeghen et al., 1992). An exploratory analysis was conducted to see if improvements vary between the types of everyday memory simulations (ecologically valid measures).

In sum, the current study was designed as a two-part study: a young adult feasibility study followed by an older adult feasibility study. As established a priori, upon completion of the young adult feasibility study we revised the study method for the older adult group using data and experiences from the young adult feasibility study. As such, we report method, results, and small discussion sections separately for the young adult sample and older adult sample below. Following the summary of both studies, we provide an overall discussion.

\section{Young Adult Study Method}

\section{Young Adult: Participants}

Recruiting. We received written, informed consent from each participant and the study was approved by the Brigham Young University (BYU) Institutional Review Board (IRB). We pre-registered our feasibility study on the Open Science Framework (OSF) prior to beginning data collection (https://osf.io/xrk28). Protocols, data, and statistics code are also posted on the OSF (osf.io/xz4ty). We used the BYU SONA program to recruit a healthy young adult sample, 
including directing students to the SONA page through class announcements, media posts with approved fliers, and word of mouth. We also contacted participants from previous studies.

Once students expressed interest on SONA, we conducted a screening phone call to determine whether or not they met study qualifications (see exclusion criteria) and recorded reasons for any exclusions. During the initial screening phone call, we clearly explained expectations of participants in order to reduce dropout rate: the number, length, and location of sessions, payment amounts and schedule, and homework routines (see original young adult script on osf.io/xz4ty). Compensation depended on whether or not participants received SONA credit. If elected, participants received 18 SONA credits after the pre-intervention test. In addition, we paid participants $\$ 10$ for the pre-intervention assessment, $\$ 10$ for each treatment session attended, and \$20 for the post-intervention assessment. For participants not receiving SONA credit, we paid $\$ 40$ for the pre-intervention assessment, $\$ 10$ for each treatment session attended, and \$20 for the post-intervention assessment. Payments were provided in separate allocations to decrease dropout rates and decrease measurement attrition: $\$ 70$ (or $\$ 40$ if they received SONA credit) at session 4 , and $\$ 60$ upon completion of the post-intervention assessment. Participants who dropped out prior to the post-intervention assessment were offered payment at the same rate for all sessions attended.

Sample sizes between 24 and 50 are needed in a feasibility study to subsequently estimate a standard deviation for a sample size calculation for a larger RCTs (Julious, 2005; Sim \& Lewis, 2012; Whitehead et al., 2016). This study aimed to include approximately 24 participants (4 groups of 6 participants) in the healthy young adult group. Considering 21-27\% of participants were expected a priori to dropout based on research and intervention groups in other studies, we initially aimed to recruit 32 young adult participants (4 groups of 8 participants; Goedendorp et 
al., 2017). We recruited in groups of approximately eight participants as group sizes between

five and nine have a stronger relationship with group members than groups with less than five or greater than nine (Burlingame et al., 2013). Our initial stop rule for data collection was at 24 completers; however, due to higher than expected dropout rates in young adults, we recruited five groups and did not reach 24 completers (39 recruited: three groups of 7, two groups of 9; 20 total treatment completers). See Figure 1 for recruitment and dropout flowchart.

\section{Figure 1}

Young Adult: Recruitment Chart

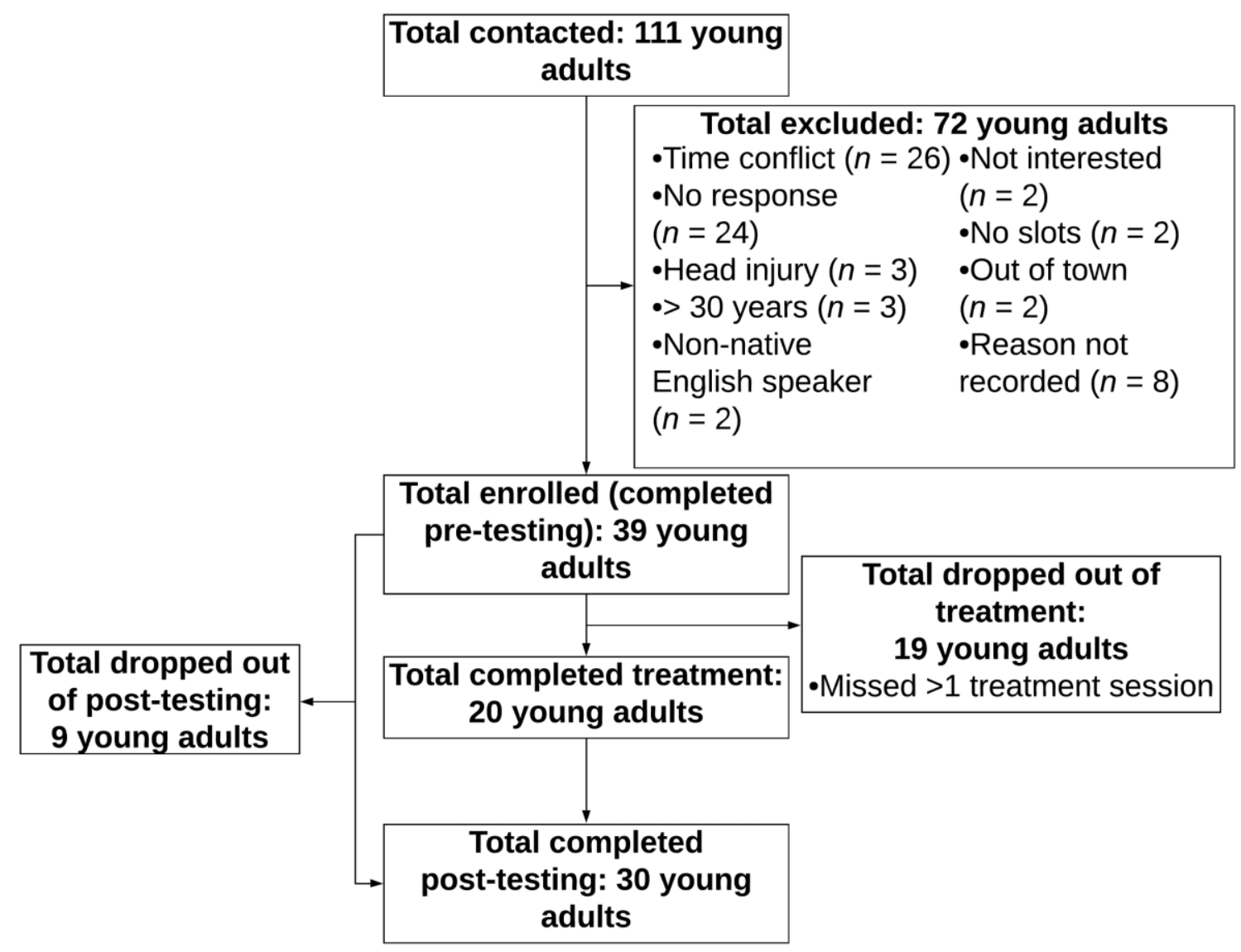

Exclusion criteria. Exclusion criteria for the healthy young adult sample (between the ages of 18 and 30) included conditions that could interfere with learning and remembering information (during assessment and intervention): reported neurological history, uncontrolled 
seizures, diagnosed psychotic conditions, bipolar disorder, learning disability, English as a second language, attention-deficit/hyperactivity disorder (ADHD), alcohol, or substance use. The initial enrolled sample of young adults ( $n=39 ; 20$ females) had an average age of 21.1 years ( $S D$ $=2.0)$ and averaged 13.9 years $(S D=1.5)$ of education.

\section{Young Adult: Procedures}

See Figure 2 for an outline of full study procedures. Each participant performed a preintervention assessment, including memory, intellectual functioning, executive functioning, emotional functioning, and group expectancy measures that took approximately three hours to complete (see Measures section below). After the pre-intervention assessment, participants attended one treatment session (maximum of 90 minutes) per week for a total of seven treatment sessions (see EON-Mem treatment protocol below). Finally, all participants performed a postintervention assessment, similar to the pre-intervention assessment with measures of memory, intellectual functioning, executive functioning, emotional functioning, and group expectancy (see Measures section below; 3 hours). 


\section{Figure 2}

Study Procedures Flowchart

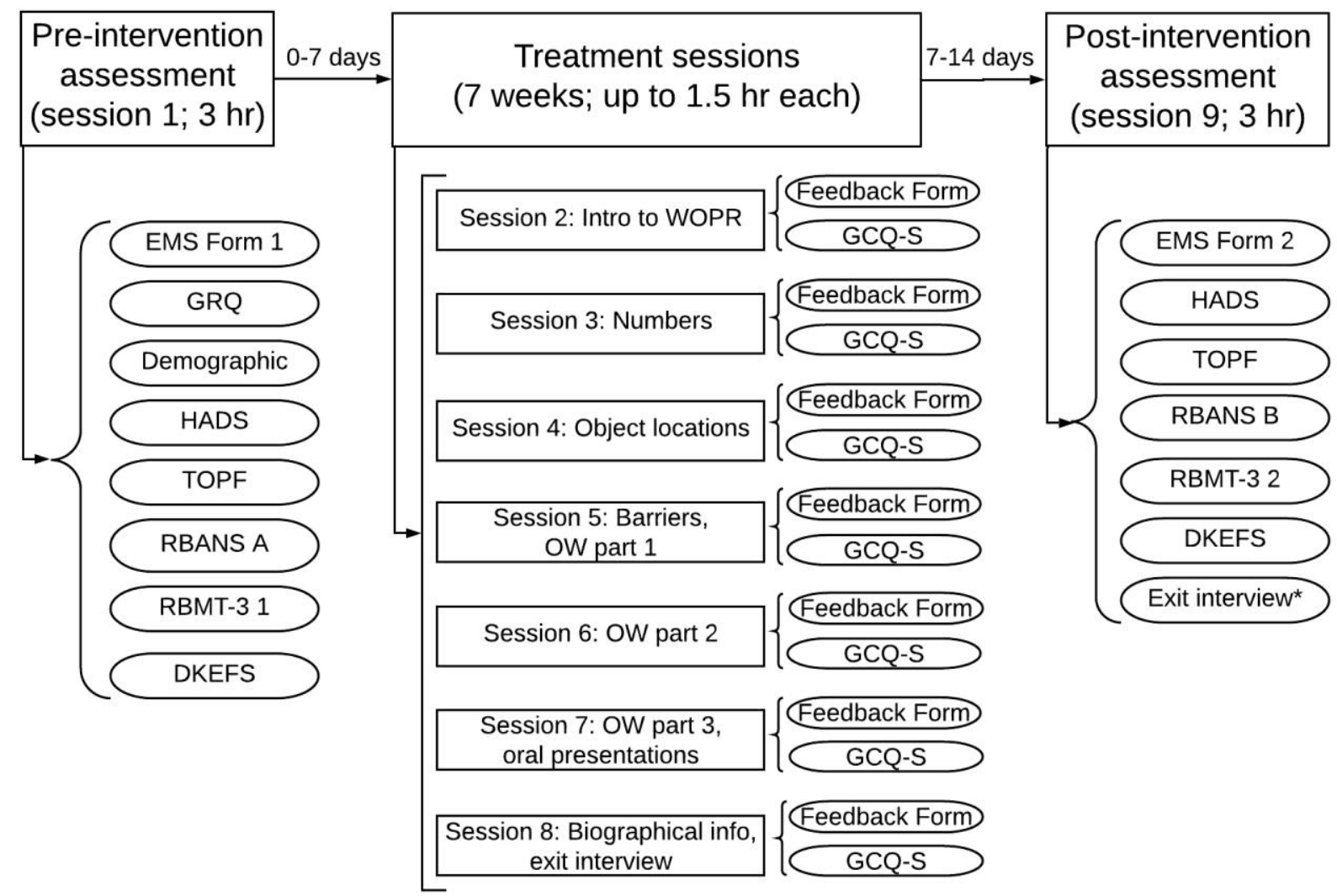

Note. EMS, Everyday Memory Simulations; GRQ, Group Readiness Questionnaire; HADS, Hospital Anxiety and Depression Scale; TOPF, Advanced Clinical Solutions: Test of Premorbid Functioning;

RBANS A, Repeatable Battery for Neuropsychological Status Update A; RBMT-3, Rivermead

Behavioral Memory Test, Third Edition; DKEFS, Delis-Kaplan Executive Function System; WOPR, write, organize, picture, rehearse; OW, Oral and Written Information; GCQ-S, Group Climate

Questionnaire, short form Engagement subscale; RBANS B, Repeatable Battery for

Neuropsychological Status Update B.

*Exit interview performed in the older adult group only.

The following treatment protocol was adapted from the one-on-one EON-Mem treatment protocol with permission from the EON-Mem author (Stringer, 2011). The EON-Mem program 
is a four-step method labeled with the acronym WOPR (write, organize, picture, rehearse) focused on teaching strategies to compensate for observed deficits in individuals with memory impairment (Stringer, 2011). Adjustments to the original manualized treatment are discussed in Table 1 (for the PowerPoint slide content see https://osf.io/xz4ty/). The first and last sessions were assessments as noted above. Thus, the numbering in Table 1 starts on session two. 


\section{Table 1}

Treatment Session Content

Session Content

Two Participants were introduced to the EON-Mem program and discussed their goals for memory improvement. Participants were introduced to the WOPR model.

Three Participants focused application of WOPR to learning numbers.

Four Participants used WOPR to remember object locations for frequently lost items.

Five Participants discussed barriers to implementation of previously learned strategies in their daily lives. As a group, participants problem solved how to overcome the barriers.

Participants were then introduced to learning oral and written information through the write and organize steps.

Six Participants learned to implement WOPR to oral and written information using organization (acronyms and acrostics) and visualization (picturing the information).

Seven Participants learned how to rehearse oral and written information. Participants reviewed the oral and written information module of learning. Participants also began a practice exercise with a story read aloud in session. Finally, participants practiced making oral presentations.

Eight Participants learned to apply WOPR to biographical information about individuals. Participants reviewed and performed troubleshooting for WOPR across all information taught over the course. The session ended with reviewing accomplishments and performing an exit interview as a group.

\section{Young Adult: Measures}

All participants performed a baseline assessment during week one (all measures and psychometric characteristics listed below), and a post-intervention assessment at week nine. The 
post-intervention assessment included the same tests as the baseline assessment using alternate versions when available.

Intelligence Quotient (IQ) estimate. Intelligence is associated with memory ability in older adults (Knight et al., 2006; Wechsler, 2001). For example, in a study of 272 older adults, mean age $=73.7(S D=5.8)$, IQ estimate scores were associated with performance on a verbal memory task, such that fewer errors on the National Adult Reading Test (NART; a word-reading test used to estimate IQ) was associated with higher scores on the verbal memory task (Knight et al., 2006). Specifically, effect sizes between estimated IQ and verbal memory ranged from $R^{2}=$ .1 to .3 depending on the individual trial (Knight et al., 2006).

In our study, we used the Test of Premorbid Functioning in the Advanced Clinical Solutions for Wechsler Adult Intelligence Scale, Fourth Edition (WAIS-IV) and Wechsler Memory Scale, Fourth Edition (WMS-IV) to obtain an estimate of IQ (Wechsler, 2009). In the Test of Premorbid Functioning, participants were asked to pronounce words that do not follow the typical grapheme to phoneme pattern (Chu et al., 2012; Wechsler, 2009). The Test of Premorbid Functioning is a revision of the Wechsler Test of Adult Reading (WTAR; Wechsler, 2001; Wechsler, 2009). Using the same normative sample as the WAIS-IV, internal reliability scores for the Test of Premorbid Functioning were above .95 and test-retest correlations were between .89-.95 (Chu et al., 2012). The Test of Premorbid Functioning is also highly correlated with other tests designed to measure verbal functioning (Chu et al., 2012). The WTAR, the original version of the Test of Premorbid Functioning, correlated strongly with scores on the national adult reading test (NART) at .9 , and with the verbal intelligence scores of the Wechsler Adult Intelligence Scale, $3^{\text {rd }}$ edition (.7-.8; Wechsler, 2001). The WTAR scores correlated 
moderately with memory scores on the Wechsler Memory Scales, $3^{\text {rd }}$ edition (.47-.51; Wechsler, 2001).

Executive functioning. Executive functioning skills are important in memory for older adults. Older adults are less likely than young adults to apply clear strategies that may require executive skills in forming memories; executive functioning likely influences one's ability to think of and apply strategy (Prull et al., 2000; Rogers et al., 2000). For example, differences in prospective memory between older adults and younger adults in a sample of 285 participants were in part explained by executive functioning abilities such as inhibition and switching $(ß=$ .20 and .15 respectively, $\mathrm{p}<.01$; Schnitzspahn et al., 2013). As such, we included two measures of executive functioning in our assessments.

First, we included the Delis Kaplan Executive Functioning System (D-KEFS) ColorWord Interference Test, a measure of inhibition (Delis et al., 2001; Karr et al., 2018). The ColorWord Interference Test required participants to first read or name colors rapidly, and then to inhibit a prepotent response (i.e., reading) by naming a color of ink rather than reading the word. The final component of the task required the individual to switch between naming the color or reading the word based on a contextual cue provided (i.e., if in a black border, read the word). The main score was based on the amount of time it took to complete each task (Delis et al., 2001). Internal consistency was moderate to high (Cronbach's $\alpha$ of .62 to .86) and test-retest reliability was moderate to high $(r=.49$ to .90 ; Delis et al., 2001)

Our second test of executive functioning was the D-KEFS Sorting Test (ST), a measure of switching or shifting (Delis et al., 2001; Karr et al., 2018). Administration of the DKEFS ST required participants to sort cards into equal groups and describe which features made the groups distinct from each other. In the second part of the DKEFS ST, participants were given a series of 
arrangements for the cards and were asked to identify and give descriptions of each group (Delis et al., 2001). The DKEFS ST had an internal consistency ranging from moderate to high (Cronbach's $\alpha$ of .55 to .82) and a moderate test-retest reliability (r of .46 to .73; Delis et al., 2001).

Depression and anxiety symptoms. We measured depression and anxiety symptoms using the Hospital Anxiety and Depression Scale (HADS; Zigmond \& Snaith, 1983) as the presence of depression and anxiety are each associated with poor memory performance (Bierman et al., 2005; Burt et al., 1995; Yochim et al., 2013). The HADS is a fourteen-item scale assessing anxious (7 items) and depressive ( 7 items) symptoms with responses ranging from 0 to 3 (Zigmond \& Snaith, 1983). Scores range from 0 to 21 for anxiety and 0 to 21 for depression (Snaith, 2003). Scores can also be combined to form a composite score (42 maximum; Zigmond $\&$ Snaith, 1983). Higher scores are indicative of more severe symptoms of the respective domains, and the recommended cut-off is greater than or equal to 8 for each domain (Zigmond \& Snaith, 1983). Psychometric properties of the HADS indicate an internal consistency as measured by Cronbach's alpha between .68 and .93 for the anxiety subscale, and between .67 and .90 for the depression subscale (Bjelland et al., 2002). Moreover, the two-factor structure was achieved in 11 studies (Bjelland et al., 2002). Area Under the Curve values were adequate ranging from 0.84-0.96 (Bjelland et al., 2002). The HADS was developed specifically to avoid somatic symptoms that may be similar across somatic and mood illnesses such as those often experienced by older adults or individuals in a hospital setting (Harter et al., 2006).

Memory. We measured memory as our primary outcome variable, both with standardized, traditional neuropsychological measures of memory (in order to improve replicability and internal validity of the study) and with everyday memory measures (in order to 
improve external validity of the study). We included the RBANS as a short standardized, traditional battery for attention, immediate memory, delayed memory, language, and visuospatial skills as well as a total score (Randolph et al., 1998; Thaler et al., 2013). In a sample of 631 older adults $(M$ age $=73.2 ; S D=5.9)$, the RBANS showed good internal consistency with a Cronbach's alpha of .86 across the six indices and inter-correlations between domains ranging from .25 to .79 (Gontkovsky et al., 2004). Moreover, the five domains accounted for 97\% of the variance of the total score (Gontkovsky et al., 2004).

We also included the Rivermead Behavioural Memory Test, Third Edition (RBMT-3) as an ecologically valid memory measure (Wilson et al., 2008). The RBMT-3 measures memory for several types of information: names, belongings, appointments, pictures, stories, faces, routes, messages, orientation and date, and novel tasks (Wilson et al., 2008). The RBMT-3 also provides a general memory index score (Wilson et al., 2008). In a sample of 80 patients with brain injury, therapist observation of memory lapses (mean $=35$ hours of observation per patient) and RBMT scores were significant ( $r=-.75$ ), suggesting good criterion validity. Further, the RBMT showed no significant differences compared to the Wechsler Memory Scale-Revised in discriminating subgroups of participants, such as those with Alzheimer's Disease, those with epilepsy, older adults, and controls (Perez \& Godoy, 1998). In the standardization study for the RBMT-3, reliability for the general memory index score was .87 for both version one and version two; inter-rater reliability for each of the subtests ranged from .79-1.0 (Wilson et al., 2008). Alternateform reliabilities for each of the RBMT-3 subtests were somewhat low, ranging from .26-.73 in the standardization sample and $.57-.86$ in a mixed sample (standardization sample plus 75 clinical cases; Wilson et al., 2008). 
Finally, we included original measures of Everyday Memory Simulations created by Stringer (2011) to test for "real-life" memory. The Everyday Memory Simulations were composed of six declarative memory (e.g., memories that can be stated such as facts or events) tasks and one prospective memory (e.g., memory for future events, such as turning off a stove in five minutes) task (Stringer, 2011). We included only five of the declarative memory tasks as one task (route finding) was not made available to us. More description for each Everyday Memory Simulation is below.

Prospective memory: Message delivery. Participants were asked to put a folded paper out of sight and read it in 10 minutes. The examiner then set a clock on the table and stated the current time. Participants were then cued to take out the paper 20 minutes later, if they had not already. The paper instructed participants to tell the examiner to "turn off the stove" in 30 minutes. If participants did not tell the examiner to turn off the stove one hour after reading the paper, they were asked by the examiner if they were supposed to tell the examiner something.

Address and phone number. Participants were given two minutes to study a phone number and address on an invitation. They were then asked to recite their own address and phone number; participants were asked to recall the phone number and address on the invitation immediately and 15 minutes after study.

Object locations simulation. Participants were given a list of 36 objects and a floor plan with six rooms and asked to place each of the objects in a room. One third of the objects did not have a typical association with a room (e.g., dog leash). Participants were given one minute to study the floor plan and then asked to recall the location for each object after removing the map immediately and 15 minutes after study. 
News article. Participants were given 2.5 minutes to study a newspaper article (allowed to write on the article) and then asked to recall the information from the news article immediately and 15 minutes after study.

Medical instructions. Participants were read instructions for a medical condition (8 sentences in length) and then asked to freely recall the details. This was repeated immediately a second time. Participants were then asked to recall the instructions 15 minutes after the second recall.

Biographical information. Participants studied 3 faces (10 seconds each) and the respective first and last name and five biographical details (e.g., job, hobbies) for each face sequentially. Participants were then asked to recall information immediately and 15 minutes after study.

To establish validity and reliability of everyday memory, Stringer (2011) piloted Everyday Memory Simulations on two samples with neurological conditions. Internal reliability of Everyday Memory Simulations ranged from .73 to .99 (Stringer, 2011). Address/phone number and prospective memory tasks correlated with the Wechsler Memory Scale (WMS) and Taylor complex figure equal to or greater than other clinical measures (e.g., recalling social security numbers, date of onset of condition, future appointments; Stringer, 2011). In the current study, internal consistency (Cronbach's alpha) for the delayed portion of each subtest administered to the young adult sample ranged from poor to excellent: message delivery 0.64 , address and phone number 0.87 , object location 0.55 , news article 0.57 , medical instructions 0.60, biographical information face-name pair 0.99, biographical information face-fact pair 0.56.

Group expectancy. Because characteristics of group members such as expectancy (i.e., hoping to improve in treatment) are related to outcome (i.e., symptom reduction), we measured 
expectancy prior to treatment using the Group Readiness Questionnaire (GRQ) Expectancy subscale (Burlingame et al., 2012). In a sample of 288 college participants, higher scores on the GRQ Expectancy scale were related to higher cohesion and engagement scores and lower dropout rates; in a second sample of 269 college counseling center students, the GRQ Expectancy significantly correlated with another measure of group readiness, Group Therapy Questionnaire, Expectations about Group scale (Burlingame et al., 2012). In the current study, we used the GRQ Expectancy measure to improve group experience and attempt to decrease attrition. According to the GRQ manual (Burlingame et al., 2012), pre-group intervention is suggested for those who score 13 or greater on the Expectancy subscale (higher Expectancy scores indicate lower expectations for the group). In our study, therapists performed a pre-group intervention using Appendix A (Expectancy Helps) of the GRQ Manual at the first treatment session for all groups (Burlingame et al., 2012). Specifically, the therapist read the general script (Appendix A) at the beginning of session one. We performed this step to maximize adherence and minimize dropout rates.

Exit interview. The exit interview was performed for the group by an assessor not involved in treatment and addressed topics such as satisfaction with treatment, frequency of sessions, duration of sessions, content presented, group size, delivery of treatment in session, homework utility, and barriers to treatment such as relationship with the therapist, group members, scheduling, and requirements for participation (Tsaousides et al., 2014). A research assistant typed participant feedback during the interview. We audio recorded the exit interview in the event that we needed a transcription. 


\section{Young Adult: Weekly Measures}

The following measures were given at each treatment session (sessions 2-8).

Participant Feedback Form. We gave participants a short feedback form after each session assessing their experience of the therapy (see Appendix B). Our feedback form is based on forms currently used in Dr. Burlingame's open trial (unpublished data). Questions included information on material presentation, format, content, and external barriers to treatment. Responses are quantitative (Likert scale ranging from 1 to 5) and free response.

Group Climate Questionnaire, short form Engagement subscale. The Group Climate Questionnaire, short form (GCQ-S) is a 12-item questionnaire that measures a member's perception of the group atmosphere and consists of three subscales: Engagement, Avoidance, and Conflict (Kivlighan \& Goldfine, 1991; MacKenzie, 1983). The Engagement subscale in part measures the perception a client has of how important the group is and how close the group members are, relating to the concept of cohesion (MacKenzie, 1983). Because cohesion is predictive of outcome across a variety of group psychotherapy studies with a medium effect size $(r=.26, d=.56)$ we used the Engagement subscale from the GCQ-S (Burlingame, McClendon, et al., 2018; Burlingame et al., 2013). In a sample of 36 participants, mean age $=24.1(S D=2.1)$, the Engagement subscale had adequate internal reliability (.94; Kivlighan \& Goldfine, 1991). From 1,150 GCQ-S responses in a sample utilizing 12 different therapy groups, the items used to calculate the Engagement subscale score demonstrated adequate factor loadings (ranging from .69 to .77 ; MacKenzie, 1983$)$.

We used the Engagement scale as part of a fidelity check to treatment. Burlingame, Whitcomb, et al. (2018) suggest merely providing the group therapist with feedback from group measures is associated with fewer relationship failures in group therapy. Thus, therapists read 
each participant's response to the GCQ-S Engagement subscale each week to improve group experience and decrease attrition rates.

Fidelity checks. To ensure therapists adhered to the same intervention, we had one therapist and one trained research assistant attend each session, the therapist leading the session and the trained research assistant marking fidelity check sheets (see https://osf.io/xz4ty/). The research assistant immediately pointed out variations from the sheet to be corrected by the therapist in session.

Manipulation checks. To track engagement and participation we recorded participant homework information each week. Specifically, we recorded how many days participants attempted homework, how many sheets were completed, and attendance of each member at each session.

\section{Young Adult: Analyses}

Data screening. All data were dually entered to limit data entry errors. If research assistants noted any discrepancy between the two data entries, they corrected the data by consulting the raw data and manuals for clarification. Further, research assistants spot-checked data for accuracy (e.g., addressed controversary in scoring methods, compared raw data and entered data). Outliers were broadly defined in our study as data points three interquartile range (IQR) beyond the median or outstanding data points as determined by visual inspection of boxplots (data points located outside of the whiskers of the boxplots; total data point outliers = 125). Thirty of the outliers were corrected due to data input/scoring errors; one data point was excluded due to administration error. Data entry, scoring method, and standard administration 
were found appropriate for all other outliers, and thus the remaining 94 outliers were left in their original state. Nineteen percent of the data was missing due to high treatment dropout rates.

Primary analyses.

Aim 1: To provide descriptive statistics about (a) adherence to treatment (e.g., attendance, homework completion), (b) cognitive functioning (e.g., estimated intelligence, general cognition, executive functioning), (c) emotional functioning (e.g., anxiety and depressive symptoms), (d) participant feedback (e.g., Participant Feedback Form, GCQ-S Engagement subscale), and (e) relevant demographic information for those who completed EON-Mem in a group-format and for those who improved after EON-Mem in a group-format, specifically to inform future randomized controlled trials. First, we described adherence to treatment for each sample as a whole. Specifically, we provided descriptive statistics (count statistics, means, ranges, and standard deviations) for attendance to treatment sessions, attendance to assessment sessions, number of homework assignments completed, and number of homework assignments completed each day. We also provided descriptive statistics of the number of participants who completed the treatment, dropped out of treatment, improved in treatment, and did not improve in treatment.

Next, we described patient-reported barriers to treatment. Specifically, we reported descriptive statistics for each question of the Participant Feedback Form and for the GCQ-S Engagement subscale score. We also used comments from the exit interview information and the Participant Feedback Form to describe patient-reported barriers. In order to organize and highlight major themes from the exit interviews, we first created a template based on the structure of the exit interview with the following categories: requirements (i.e., frequency/length of sessions, scheduling, other), content (i.e., strategies), relationships (i.e., therapist, group, size), 
treatment delivery/structure (i.e., therapist, PowerPoint, group setting), and homework (utility, frequency). We then read through each exit interview and listed comments in each category. For repeated comments, we tallied the number of times the comment was stated. We also entered elaborations below general comments. We described comments as barriers, facilitators, or mixed feedback. Finally, we reported the comments with the highest frequency (for each barriers, facilitators, and mixed feedback) in tables and noted the number of times the comment was made on either a Participant Feedback Form or in the exit interview. We used elaborations from each comment to provide examples and nuance to comments within the text of the results section. Comments provided from each Participant Feedback Form were listed per group, per session for each sample. Identical comments were not repeated in the table.

Next, we obtained descriptive statistics of and effect sizes between those who completed treatment and those who dropped out of treatment for the following domains: adherence to treatment, cognitive functioning, emotional functioning, participant feedback, and relevant demographic information. We followed the Rebok et al. (2014) definition of treatment completion ( $80 \%$ attendance) and considered six or greater sessions as a successful completion of treatment in our study. Specifically, we provided descriptive statistics about participants' attendance, homework (i.e., number of assignments, and number of assignments per day), estimated premorbid IQ, baseline cognitive functioning (i.e., RBANS general cognition index score, DKEFS inhibition score, DKEFS inhibition/switching score, DKEFS ST combined description score), anxiety and depressive symptoms (HADS depression score, HADS anxiety score), GRQ Expectancy subscale score, GCQ-S Engagement subscale score, age, education, and sex for participants who completed treatment and participants who dropped out of treatment. We recorded the same descriptive statistics for, and calculated effect sizes between, participants who 
showed improvement and who did not (according to the Jacobson and Truax (1991) reliable change indices method see Aim 3 for details). Finally, we recorded the same descriptive statistics and effect sizes for those that completed their homework, at two cut-off rates: $50 \%$ and $75 \%$.

Notably, we did not perform any statistical comparisons between the young adult and older adult samples because the study is a feasibility study and not a clinical trial. Thus, we could not state any significant differences or make any statistical inferences between samples. As explained above, we instead performed cross-tabulation and calculated the effect sizes on several variables between groups (completer vs. non-completer, improver vs. non-improver, $<50 \%$ homework vs. $50-75 \%$ homework vs. $>75 \%$ homework) within each sample to help determine potentially important variables and aims to consider in the analyses performed for future trials. Importantly, the effect sizes reported in the study should not be described or interpreted in the same way as in an inferential setting nor should they be used to infer the existence of relationships between variables in a more general population.

Aim 2: To provide information regarding resources required for $\mathrm{EON}-\mathrm{Mem}$. First, we described the cost required for implementation. We calculated the average cost of protocols and reimbursement per participant. We summed time spent on scoring/data entry, recruiting, and training research assistants for each sample. We calculated average time for a pre-intervention test, post-intervention test, and session (across all sessions and per specific session number). We then calculated average cost and average research time required for a participant. In regard to recruitment, we reported the number of participants contacted for the study and the number of participants excluded from the study with accompanying reasons for exclusion.

Aim 3: To assess change in memory performance for healthy young adults and older adults from pre-EON-Mem training to post-EON-Mem training. For all cognitive measures, we 
assessed change from pre/post treatment using reliable change indices (RCI) as defined by Jacobson and Truax (1991); in particular, we used the corrected and adopted version by Christenson and Mendoza (Christensen \& Mendoza, 1986; Wise, 2004). The RCI was conducted for each individual in each sample (older adult, young adult), separately, by assessing pre- versus post- performance on "Delayed Address and Phone Number Total Points," "Delayed Object Locations Total Points," "Delayed Medical Instructions Total Points," "Delayed Newspaper Article Total Points," "Biographical Information: Sum of Delayed Faces and Names Total Points," "Biographical Information: Sum of Delayed Facts Total Points," "Message Delivery Content Score," the RBANS delayed memory, and Rivermead General Memory Index. Post-hoc, we chose to also perform RCI for each RBANS subtest (the non-memory subtests). In addition to measurement of the outcome variable at two timepoints, the RCI equation required a standard deviation and reliability. We calculated standard deviations of the measures from the preintervention test scores in our own respective samples (reliabilities for young adults and older adults). Where available, we used reliability coefficients from manuals that were most similar to the age of our sample and timeline in our study (RBANS, RBMT). Because neither alternateforms reliability nor test-retest reliability were available from previous research on Everyday Memory Simulations, we calculated and used the internal reliabilities of each Everyday Memory Simulation at pre-intervention testing in our respective samples (reliabilities for young adults and older adults). We chose to use internal reliability as opposed to test-retest reliability because the intervention performed between testing sessions invalidated the statistic. As such, the use of internal reliability as a substitute for test-retest reliability was an assumption, and potential limitation, of this study (see Table 2 for exact reliability values). 
Table 2

Young Adult: Reliabilities for Calculating Reliable Change Indices

\begin{tabular}{|c|c|c|}
\hline Test & Source of Reliability & Coefficient \\
\hline Delayed Address and Phone Number Total Points & Current sample: Cronbach's alpha & 0.87 \\
\hline Delayed Object Locations Total Points & Current sample: Cronbach's alpha & 0.55 \\
\hline Delayed Medical Instructions Total Points & Current sample: Cronbach's alpha & 0.60 \\
\hline Delayed Newspaper Article Total Points & Current sample: Cronbach's alpha & 0.57 \\
\hline Bio: Sum of Delayed Faces and Names Total Points & Current sample: Cronbach’s alpha & 0.99 \\
\hline Bio: Sum of Delayed Facts Total Points & Current sample: Cronbach's alpha & 0.56 \\
\hline Message Delivery Content Score & Current sample: Cronbach's alpha & 0.64 \\
\hline RBANS delayed memory & Manual: Test-retest coefficient & 0.80 \\
\hline Rivermead General Memory Index & Manual: Reliability coefficient & 0.87 \\
\hline RBANS List Learning & Manual: Test-retest coefficient & 0.66 \\
\hline RBANS Story Memory & Manual: Test-retest coefficient & 0.72 \\
\hline RBANS Figure Copy & Manual: Test-retest coefficient & 0.57 \\
\hline RBANS Line Orientation & Manual: Test-retest coefficient & 0.72 \\
\hline RBANS Picture Naming & Manual: Test-retest coefficient & 0.73 \\
\hline RBANS Semantic Fluency & Manual: Test-retest coefficient & 0.65 \\
\hline RBANS Digit Span & Manual: Test-retest coefficient & 0.67 \\
\hline RBANS Coding & Manual: Test-retest coefficient & 0.79 \\
\hline RBANS List Delay & Manual: Test-retest coefficient & 0.66 \\
\hline RBANS List Recognition & Manual: Test-retest coefficient & 0.70 \\
\hline RBANS Story Memory Delay & Manual: Test-retest coefficient & 0.49 \\
\hline RBANS Figure Delay & Manual: Test-retest coefficient & 0.71 \\
\hline
\end{tabular}


In order to understand how using internal reliability for the Everyday Memory Simulation analyses may have impacted results, we performed a sensitivity analysis substituting a reliability coefficient from a comparable memory measure (the RBMT) in the RCI equation. We found small differences in the young adult results overall using the two different reliability coefficients (using the Everyday Memory Simulations internal reliability coefficient: 9\% improved, 81\% did not change, $11 \%$ performed worse; using the RBMT reliability coefficient, $14 \%$ improved; $75 \%$ did not change; $10 \%$ performed worse). Overall, the sensitivity analysis provides support for using the Everyday Memory Simulations internal reliabilities to calculate the RCI.

As the study was not an RCT with sufficient power for inferential statistical analyses, estimates of change were interpreted with caution and we simply reported patterns of change for individuals in the group, rather than focusing on $p$-values, although memory scores were expected to change (improve) over time on both standardized and everyday tests of memory.

\section{Young Adult: Results}

\section{Aim 1: To Provide Descriptive Statistics about Adherence to Treatment, Cognitive and Emotional Functioning, Participant Feedback, and Relevant Demographic Information}

For information regarding treatment attendance and homework completion for the young adult sample as a whole, see Table 3. Overall, only 20 of the 39 young adult participants (51\%) completed treatment, averaging $4.6(S D=2.4)$ of seven possible treatment sessions $(66 \%$ of all treatment sessions; see Figure 3 for treatment sessions attendance). Three of the 39 participants $(8 \%)$ did not attend any treatment sessions, dropping out of treatment immediately following the pre-intervention assessment. Testing attrition was somewhat lower than treatment attrition: 30 of the 39 participants (77\%) attended the post-intervention assessment (see Figure 3 ). Of note, 10 of the 19 participants $(53 \%)$ who did not complete treatment returned for post-intervention testing. 
Table 3

Young Adult: Description of Attendance and Homework for the Sample

\begin{tabular}{|c|c|c|c|}
\hline Variable & $n$ & Mean $(S D) /$ Percentage & Range \\
\hline Treatment completion category $(6+$ sessions $)$ & 39 & & \\
\hline Completed & 20 & 51.3 & \\
\hline Did not complete & 19 & 48.7 & \\
\hline Treatment attendance (number of sessions) & 39 & $4.6(2.4)$ & $0-7$ \\
\hline 0 & 3 & 7.7 & \\
\hline 1 & 4 & 10.3 & \\
\hline 2 & 2 & 5.1 & \\
\hline 3 & 2 & 5.1 & \\
\hline 4 & 3 & 7.7 & \\
\hline 5 & 5 & 12.8 & \\
\hline 6 & 10 & 25.6 & \\
\hline 7 & 10 & 25.6 & \\
\hline Testing attendance (number of sessions) & 39 & $1.8(0.4)$ & $1-2$ \\
\hline 1 & 9 & 23.1 & \\
\hline 2 & 30 & 76.9 & \\
\hline Homework assignments (number completed) & 29 & $36.3(22.0)$ & $0-70$ \\
\hline $0-9$ & 6 & 20.7 & \\
\hline $10-19$ & 1 & 3.5 & \\
\hline $20-29$ & 2 & 6.9 & \\
\hline $30-39$ & 5 & 17.2 & \\
\hline $40-49$ & 4 & 13.8 & \\
\hline $50-59$ & 6 & 20.7 & \\
\hline $60-69$ & 1 & 3.5 & \\
\hline $70-77$ & 2 & 6.9 & \\
\hline Days worked on homework (days) & 26 & $21.5(14.3)$ & $0-49$ \\
\hline $0-7$ & 6 & 23.1 & \\
\hline $8-14$ & 2 & 7.7 & \\
\hline $15-21$ & 5 & 19.2 & \\
\hline $22-28$ & 6 & 23.1 & \\
\hline $29-35$ & 3 & 11.5 & \\
\hline $36-42$ & 1 & 3.9 & \\
\hline $43-49$ & 3 & 11.5 & \\
\hline Improved category & 30 & & \\
\hline Improved (RCI 1.96+) & 6 & 20.0 & \\
\hline Did not improve $(\mathrm{RCI}<1.96)$ & 24 & 80.0 & \\
\hline
\end{tabular}

Note. RCI, reliable change indices. 


\section{Figure 3}

Young Adult: Session Attendance

A Treatment Session Attendance

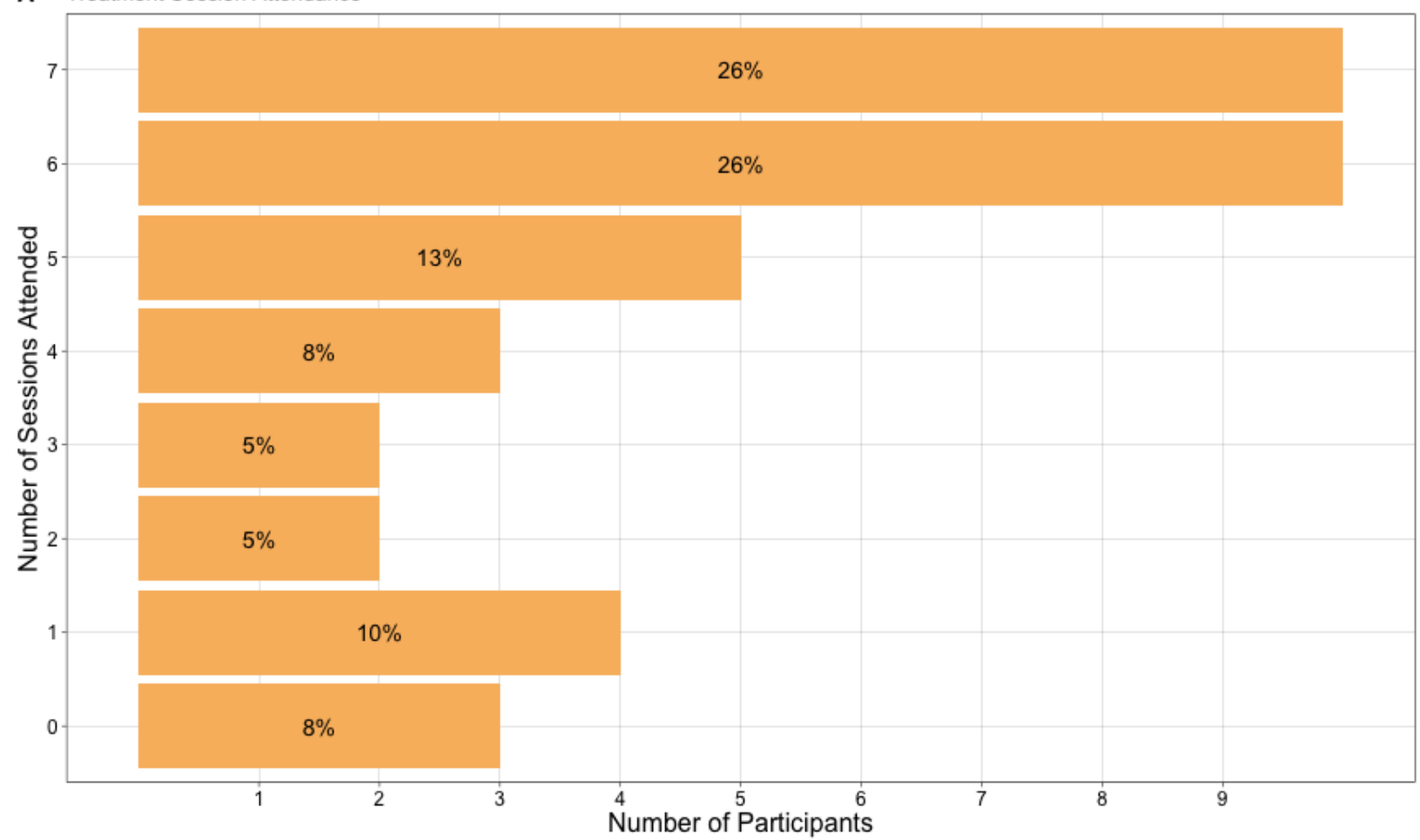

B Testing Session Attendance

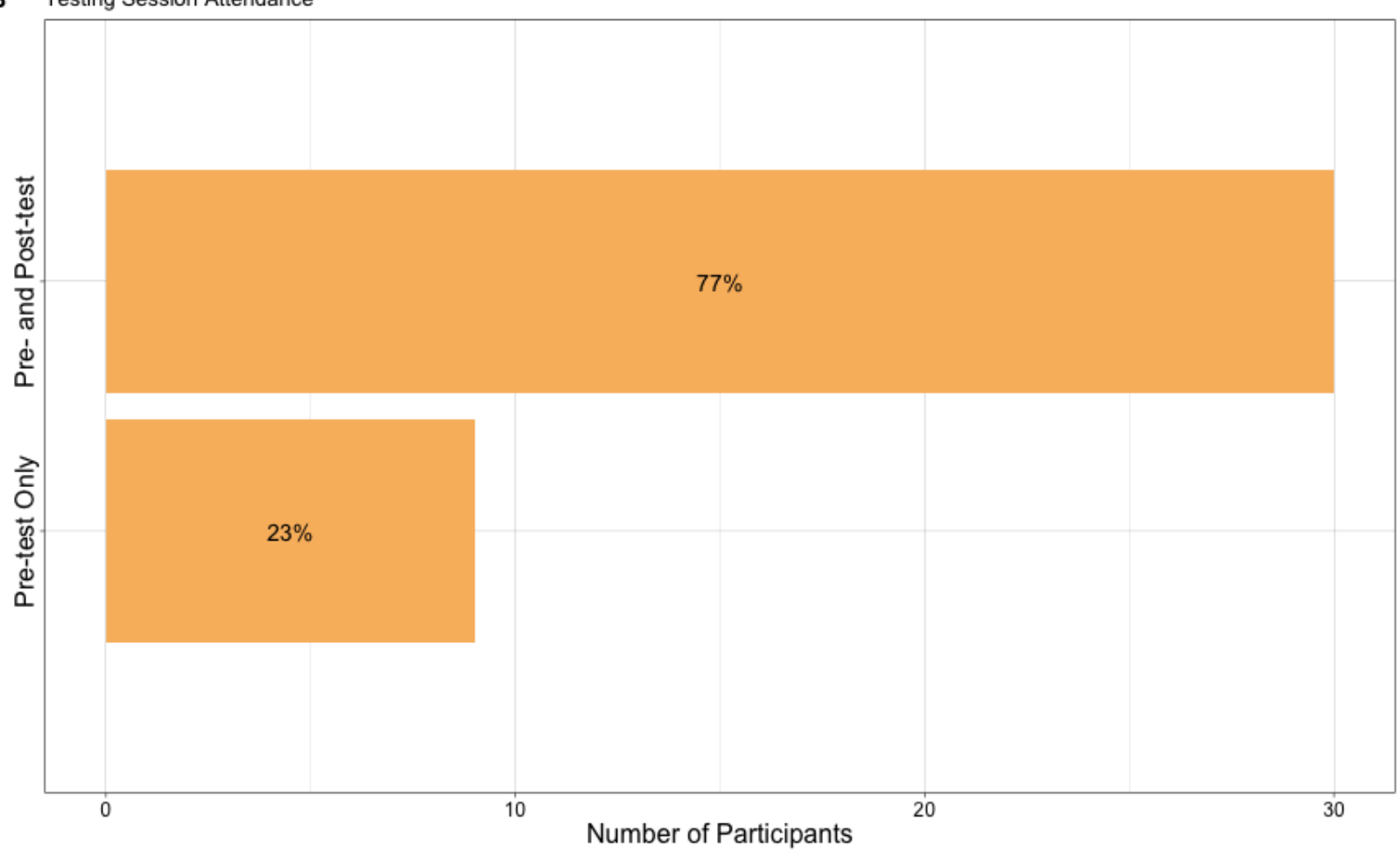


The young adult group had low rates of homework completion: participants on average completed only $36.3(S D=22.0)$ of 77 possible homework assignments $(47 \%$ of all homework assigned). The young adults worked on homework an average of $21.5(S D=14.3)$ of 49 possible days in treatment (44\% of the total days in treatment; see Figure 4). 


\section{Figure 4}

\section{Young Adult: Homework Completion}

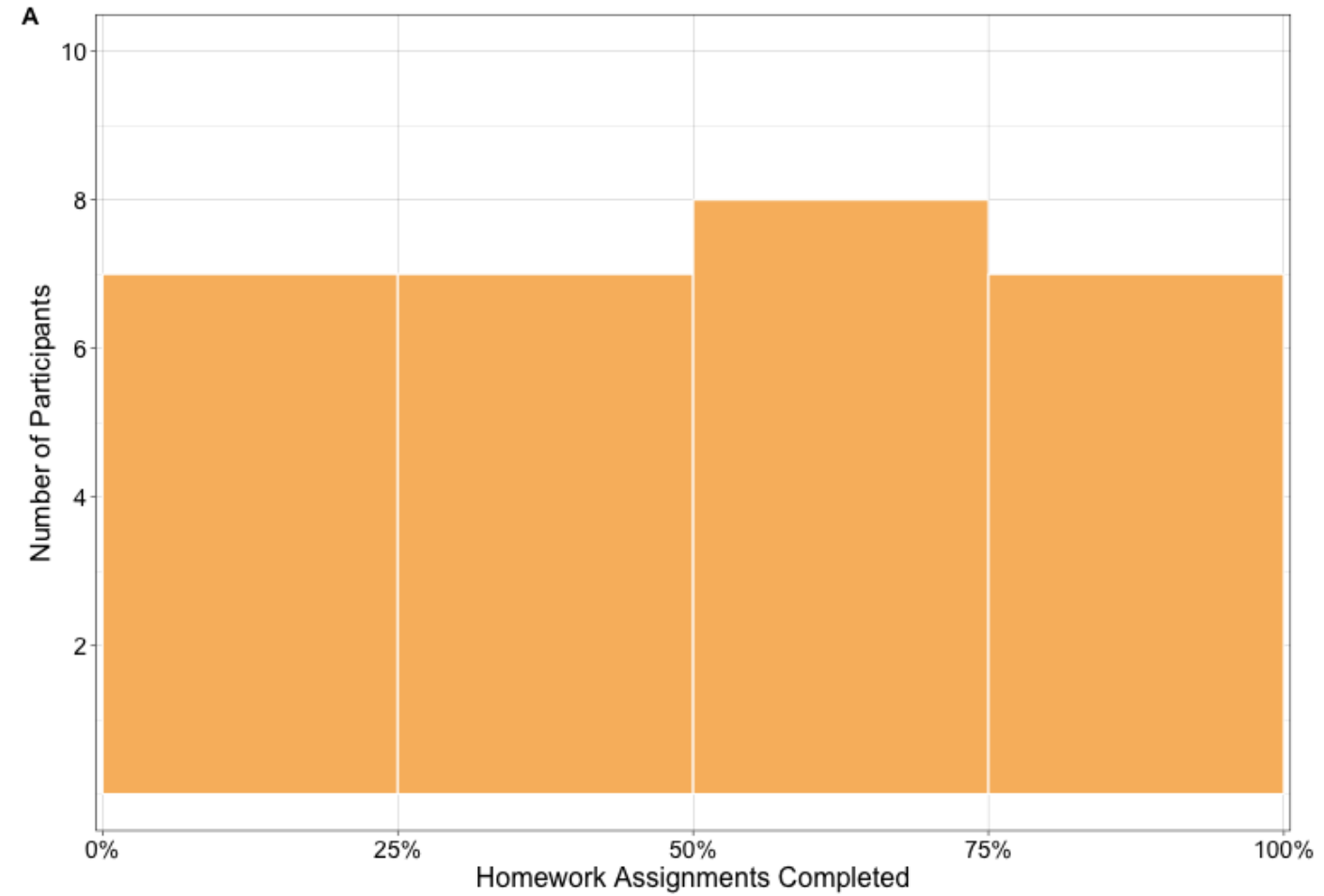

B Time Spent on Homework

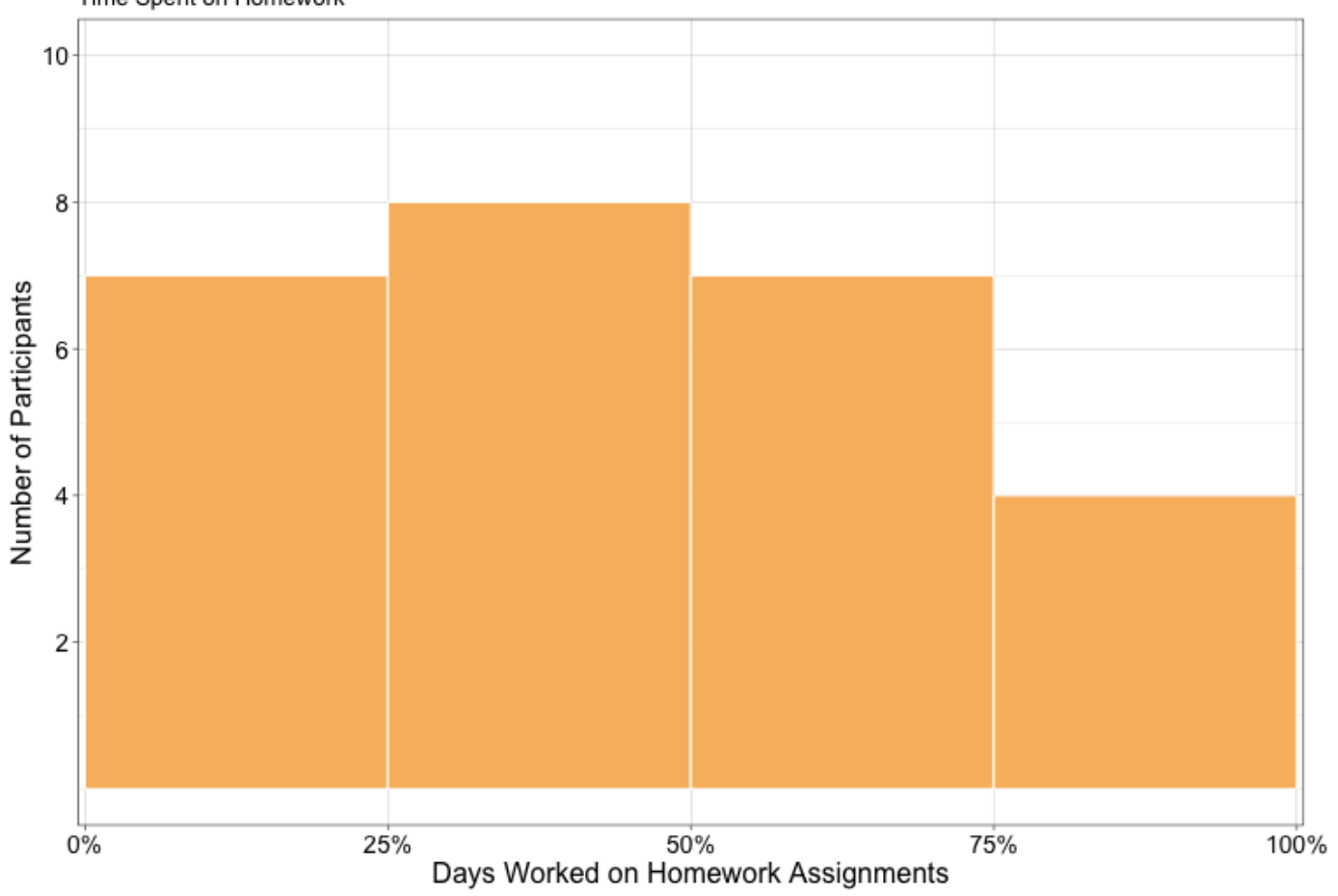


For tables regarding the young adult general quantitative and qualitative participantreported facilitators and barriers to treatment, see Tables 4 and 5. For more detailed session-bysession feedback from young adult participants, see Supplemental Tables 1 and 2 on OSF (osf.io/xz4ty). Supplemental Table 1 includes facilitators and barriers described by young adult participants on their weekly Participant Feedback Form for each of the seven treatment sessions. Supplemental Table 2 includes average scores for each question on the Participant Feedback Form and for each question on the GCQ-S Engagement subscale for each of the seven treatment sessions. 
Table 4

Young Adult: Quantitative Participant Feedback from Weekly Treatment Session Questionnaires

\begin{tabular}{lll}
\hline Variable & Mean $(S D) /$ Percentage & Range \\
\hline GCQ-S Engagement subscale: Total & $3.1(1.0)$ & $1.0-4.9$ \\
\hline Question 1: liked each other. & $3.7(1.0)$ & $2.0-5.6$ \\
Question 2: tried to understand. & $3.5(1.1)$ & $1.0-5.6$ \\
Question 3: sense of participation. & $4.1(0.9)$ & $2.0-6.0$ \\
Question 4: confront each other. & $2.5(1.3)$ & $0.0-4.8$ \\
Question 5: reveal sensitive info. & $1.5(1.4)$ & $0.0-5.0$ \\
\hline Participant Feedback Form & & $1.0-3.0$ \\
\hline Question 1: training useful. & $1.7(0.5)$ & $1.0-2.5$ \\
Question 2: education clear. & $1.3(0.4)$ & $1.0-2.5$ \\
Question 3: presentation helpful. & $1.6(0.4)$ & $1.0-3.0$ \\
Question 4: group practice helpful. & $1.7(0.5)$ & $1.0-2.3$ \\
Question 5: therapist clear. & $1.2(0.4)$ & $1.0-4.0$ \\
Question 6: homework useful. & $2.2(0.6)$ & $2.3(0.8)$ \\
Question 7: no barriers to homework. & $2.3(0.7)$ & \\
Question 8: training helped in life. & & \\
\hline
\end{tabular}

Note. GCQ-S, Group Climate Questionnaire, short form.

\begin{tabular}{|c|c|}
\hline GCQ-S Key & $\frac{\text { Participant Feedback }}{\text { Form Key }}$ \\
\hline Not at all: 0 & \\
\hline A little bit: 1 & Strongly agree: 1 \\
\hline Somewhat: 2 & Somewhat agree: 2 \\
\hline Moderately: 3 & Do not agree/disagree: 3 \\
\hline Quite a bit: 4 & Somewhat disagree: 4 \\
\hline A great deal: 5 & Strongly disagree: 5 \\
\hline Extremely: 6 & \\
\hline
\end{tabular}




\section{Table 5}

Young Adult: Reported Facilitators and Barriers to EON-Mem Treatment

\begin{tabular}{c} 
Themes \\
\hline Facilitators \\
\hline Relationships: therapist and group members \\
Presentation: organized, clear, good pace \\
Reviewing homework \\
Apathy: unmotivated, inapplicable \\
Content on peg words: difficult, dislike \\
Reminder texts: variable/poor timing \\
Homework: unmotivated, inapplicable, not worth it \\
\hline Attendance requirements: time, frequency, scheduling \\
Content of sessions \\
\hline
\end{tabular}

Young adults list a few facilitators that helped their treatment. First, participants appreciated relationships formed throughout the treatment (15 positive comments made regarding relationships; also, see responses to questions one through three of the GCQ-S Engagement subscale in Figure 5): they got along well with other members and the therapist, liked the number of participants in each group, and enjoyed the group dynamic. Although most participants appreciated the small group size and group dynamic, three comments indicated a larger group size would be desirable; those who desired a larger group size came from groups with high dropout rates and attendance as low as two-to-three members at some sessions. Second, the young adults felt the presentation of information was clear, organized, easy to 
follow, and an appropriate pace (14 related comments; also, see response to questions two, three, and five of the Participant Feedback Form in Figure 6). Third, and finally, the young adults appreciated reviewing details of their homework together in class (six related comments). 
Figure 5

Young Adult: GCQ-S Engagement Subscale

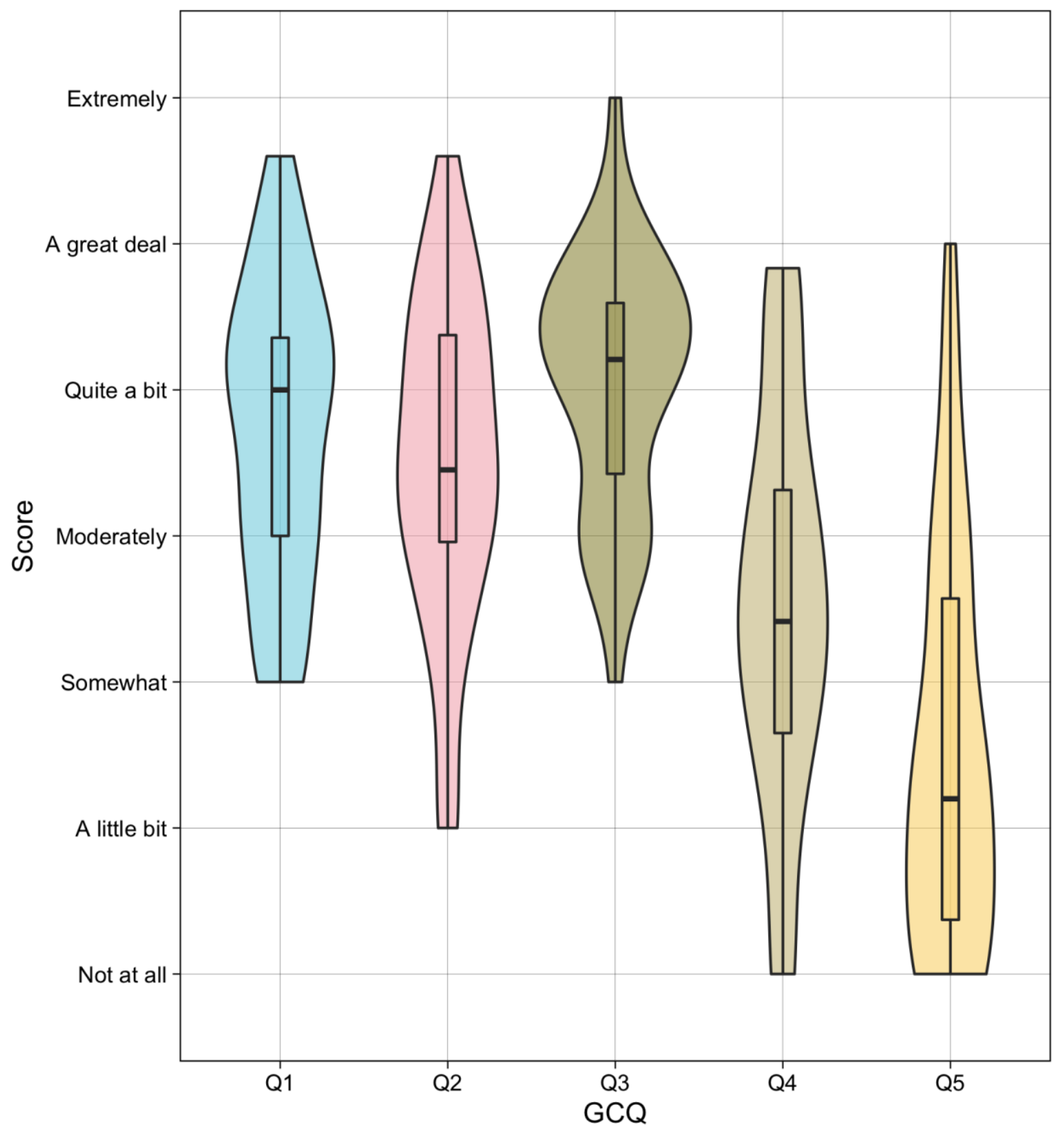

Note. GCQ-S: Group Climate Questionnaire, short form

Q1: The members liked and cared about each other.

Q2: The members tried to understand why they do the things they do, tried to reason it out.

Q3: The members felt what was happening was important and there was a sense of participation.

Q4: The members challenged and confronted each other in their efforts to sort things out.

Q5: The members revealed sensitive personal information or feelings. 
Figure 6

Young Adult: Participant Feedback Form

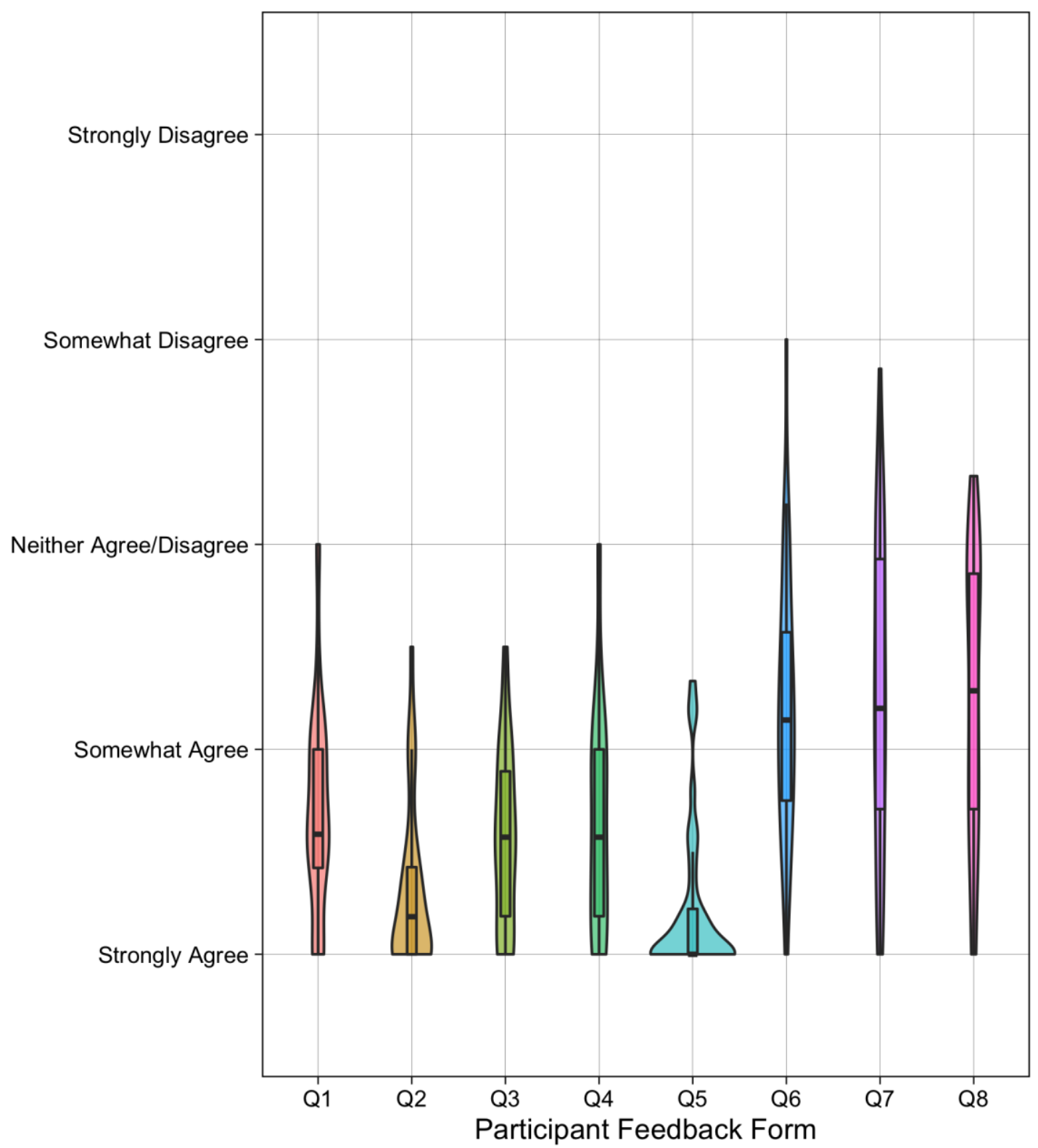

Note. Q1: The training was useful.

Q2: The educational portion was clear.

Q3: I found the presentation helpful.

Q4: The in-group practices were helpful.

Q5: The therapist was clear and easy to follow.

Q6: The homework was useful.

Q7: I did not encounter barriers to completing the homework.

Q8: The training helped me in everyday life. 
Young adults also communicated barriers they experienced in treatment. Young adult participants generally felt apathetic toward treatment: many noted their memory or strategies for memory were adequate and they were thus unmotivated to participate in treatment. Others noted the treatment was not applicable to them, especially if they were not currently attending school (seven related comments; see relatively wider distribution in responses to question eight on the Participant Feedback Form, Figure 6). Next, the young adults did not like some of the content: for example, several participants disliked peg words and considered peg words too complicated and too difficult (seven total comments, five of which were specific to peg words). Young adults also felt reminder texts for homework and sessions were too variable in their timing and often given at a poor time to help them remember to complete their homework (four related comments). Lastly, they felt unmotivated to do the homework; many participants felt the benefits from the homework did not outweigh the cost of spending time to complete the homework and found it difficult to find applicable content to study for the homework (seven related comments; see wide range in distribution on questions six and seven of the Participant Feedback Form).

The young adults reported mixed feedback on requirements for attendance, compensation, and content of treatment sessions. Specifically, young adults were variable in how they felt about the requirements to attend: some stated the scheduling and duration of sessions were hard to commit to whereas others had no problem (six negative comments, 13 positive comments). Some young adults felt they were compensated more than enough whereas others felt the compensation was not worth the cost of participating. Young adults also gave mixed feedback on content of sessions: some remarked they generally liked the session content, whereas others disliked specific sections, such as peg words, objects locations, and picturing information (seven negative comments, four positive comments). 
Cognitive and emotional functioning, demographic factors, feedback, and fidelity to treatment between various groups within the young adult sample are given in Tables 6-8. Within the following section, we will use comparative words such as "differences," "than," "higher," and "lower" to describe the data. Unlike in inferential data, differences and trends described do not implicate statistically significant differences or predictions, they are simply descriptors for understanding the data. All differences in pre-treatment variables between young adult subgroups can be observed in Tables 6-8. Below, we note variables in which group effect sizes are 0.5 or greater within our sample. In our sample, young adult completers compared to noncompleters had more males than females, higher intelligence, higher overall cognitive skills, higher inhibition skills, lower anxiety symptoms, lower depressive symptoms, higher group cohesion, and higher homework completion. In our sample, young adult improvers compared to non-improvers had more females, lower intelligence, lower cognitive skills, lower inhibition and switching skills, higher group cohesion, higher homework completion, and were younger. None of the variables demonstrated an effect size of 0.5 or greater on homework completion groups, except of course the amount of homework completed by participants. 


\section{Table 6}

Young Adult: Baseline Information for Completers in the Young Adult Sample

\begin{tabular}{lllll}
\hline Variable & $\begin{array}{l}\text { Completed } \\
(n=20)\end{array}$ & $\begin{array}{l}\text { Dropped out } \\
(n=19)\end{array}$ & Cohen's $d$ & $95 \%$ Confidence Interval \\
\hline Age & $21.2(1.6)$ & $21.0(2.3)$ & -0.1 & {$[-0.7,0.5]$} \\
Gender (\% female) & $40 \%$ & $63 \%$ & -0.5 & {$[-1.1,0.2]$} \\
Education & $13.8(1.3)$ & $14.0(1.6)$ & 0.1 & {$[-0.5,0.8]$} \\
TOPF & $121.0(7.9)$ & $118.0(6.3)$ & -0.5 & {$[-1.1,0.2]$} \\
RBANS A total index & $107.0(12.0)$ & $100.0(13.0)$ & -0.5 & {$[-1.2,0.1]$} \\
DKEFS inhibition & $13.0(1.6)$ & $12.0(2.2)$ & -0.8 & {$[-1.4,-0.1]$} \\
DKEFS inhibition/switching & $12.0(1.6)$ & $11.0(1.8)$ & -0.1 & {$[-0.7,0.6]$} \\
DKEFS sorting & $12.0(2.6)$ & $12.0(2.6)$ & -0.3 & {$[-0.9,0.4]$} \\
HADS anxiety & $5.3(2.7)$ & $7.6(3.4)$ & 0.8 & {$[0.1,1.4]$} \\
HADS depression & $2.2(2.2)$ & $3.7(3.1)$ & 0.6 & {$[-0.1,1.2]$} \\
GRQ-E & $7.9(1.7)$ & $8.4(2.1)$ & 0.2 & {$[-0.4,0.9]$} \\
GCQ-S Engagement subscale & $3.3(0.8)$ & $2.8(1.1)$ & -0.5 & {$[-1.2,0.1]$} \\
HW assignments & $50.0(14.0)$ & $20.0(19.0)$ & -1.8 & {$[-2.6,-0.9]$} \\
Days worked on HW & $29.0(11.0)$ & $13.0(13.0)$ & -1.3 & {$[-2.2,-0.5]$} \\
Session attendance & $6.5(0.5)$ & $2.7(1.9)$ & -2.8 & {$[-3.6,-1.9]$} \\
\hline
\end{tabular}

Note. HW, homework; TOPF, Advanced Clinical Solutions: Test of Premorbid Functioning; RBANS A, Repeatable Battery for Neuropsychological Status Update A; DKEFS, Delis-Kaplan Executive Function System; HADS, Hospital Anxiety and Depression Scale; GRQ, Group Readiness Questionnaire, Expectancy subscale; GCQ-S, Group Climate Questionnaire, short form. 


\section{Table 7}

Young Adult: Baseline Information for Improvers in the Young Adult Sample

\begin{tabular}{lllll}
\hline Variable & $\begin{array}{l}\text { Improved } \\
(n=6)\end{array}$ & $\begin{array}{l}\text { Did not improve } \\
(n=24)\end{array}$ & Cohen's $d$ & $\begin{array}{l}95 \% \text { Confidence } \\
\text { Interval }\end{array}$ \\
\hline Age & $19.8(1.7)$ & $21.3(1.8)$ & 0.8 & {$[-0.1,1.7]$} \\
Gender (\% female) & $83 \%$ & $37.5 \%$ & -0.5 & {$[-1.1,0.2]$} \\
Education & $13.3(1.5)$ & $13.9(1.5)$ & 0.4 & {$[-0.5,1.3]$} \\
TOPF & $115.3(6.0)$ & $120.7(7.6)$ & 0.7 & {$[-0.2,1.6]$} \\
RBANS A total index & $94.0(7.9)$ & $106.9(12.8)$ & 1.1 & {$[0.0,2.1]$} \\
DKEFS inhibition & $11.2(1.3)$ & $12.9(2.0)$ & 0.9 & {$[-0.1,1.9]$} \\
DKEFS inhibition/switching & $10.8(2.2)$ & $11.6(1.5)$ & 0.5 & {$[-0.5,1.5]$} \\
DKEFS sorting & $11.3(2.6)$ & $12.3(2.5)$ & 0.4 & {$[-0.5,1.3]$} \\
HADS anxiety & $6.7(5.4)$ & $6.0(2.7)$ & -0.2 & {$[-1.1,0.7]$} \\
HADS depression & $2.5(4.2)$ & $3.0(2.6)$ & 0.2 & {$[-0.7,1.1]$} \\
GRQ-E & $8.0(1.3)$ & $8.4(1.8)$ & 0.2 & {$[-0.7,1.1]$} \\
GCQ-S Engagement subscale & $3.4(0.7)$ & $3.1(1.0)$ & -0.5 & {$[-1.2,0.1]$} \\
HW assignments & $55.5(10.5)$ & $39.6(16.4)$ & -1.0 & -0.9 \\
Days worked on HW & $34.0(10.3)$ & $23.8(11.0)$ & -0.4 & {$[-2.0,-0.1]$} \\
Session attendance & $6.2(1.6)$ & $5.5(1.5)$ & {$[-1.3,0.5]$}
\end{tabular}

Note. HW, homework; TOPF, Advanced Clinical Solutions: Test of Premorbid Functioning; RBANS A, Repeatable Battery for

Neuropsychological Status Update A; DKEFS, Delis-Kaplan Executive Function System; HADS, Hospital Anxiety and Depression Scale; GRQ, Group Readiness Questionnaire, Expectancy subscale; GCQ-S, Group Climate Questionnaire, short form. 


\section{Table 8}

Young Adult: Baseline Information for Homework Completion Groups in the Young Adult Sample

\begin{tabular}{|c|c|c|c|c|}
\hline Variable & $\begin{array}{l}\mathrm{HW}<50 \% \\
(n=13)\end{array}$ & $\begin{array}{l}\text { HW } 50 \%-74 \% \\
(n=9)\end{array}$ & $\begin{array}{l}\text { HW } 75 \%+ \\
(n=7)\end{array}$ & $\eta_{p}^{2}$ \\
\hline Age & $21.0(2.0)$ & $21.4(1.6)$ & $21.0(1.9)$ & 0.0 \\
\hline Gender ( $\%$ female $)$ & $54 \%$ & $56 \%$ & $57 \%$ & 0.0 \\
\hline Education & $14.0(1.9)$ & $14.0(1.2)$ & $14.0(1.4)$ & 0.0 \\
\hline TOPF & $123.0(6.1)$ & $120.3(8.1)$ & $116.0(2.9)$ & 0.2 \\
\hline RBANS A total index & $101.0(12.0)$ & $103.2(18.4)$ & $105.0(11.0)$ & 0.0 \\
\hline DKEFS inhibition & $11.0(2.3)$ & $12.9(1.9)$ & $13.0(2.0)$ & 0.2 \\
\hline DKEFS inhibition/switching & $11.0(1.7)$ & $11.4(1.5)$ & $12.0(1.7)$ & 0.1 \\
\hline DKEFS sorting & $12.0(2.7)$ & $12.2(2.5)$ & $13.0(3.0)$ & 0.0 \\
\hline HADS anxiety & $6.2(2.9)$ & $6.2(4.1)$ & $6.4(3.3)$ & 0.0 \\
\hline HADS depression & $2.3(2.2)$ & $2.9(4.2)$ & $2.3(2.4)$ & 0.0 \\
\hline GRQ-E & $8.8(2.3)$ & $8.0(1.7)$ & $7.6(1.6)$ & 0.1 \\
\hline GCQ-S Engagement subscale & $2.9(1.0)$ & $3.3(0.7)$ & $3.6(0.9)$ & 0.1 \\
\hline HW assignments & $17.0(15.0)$ & $44.6(6.0)$ & $62.0(5.6)$ & 0.8 \\
\hline Days worked on HW & $12.0(10.0)$ & $25.1(5.8)$ & $41.0(9.3)$ & 0.6 \\
\hline Session attendance & $3.4(2.6)$ & $5.8(1.5)$ & $66.0(0.5)$ & 0.4 \\
\hline
\end{tabular}

Note. HW, homework; TOPF, Advanced Clinical Solutions: Test of Premorbid Functioning; RBANS A, Repeatable Battery for

Neuropsychological Status Update A; DKEFS, Delis-Kaplan Executive Function System; HADS, Hospital Anxiety and Depression Scale; GRQ,

Group Readiness Questionnaire, Expectancy subscale; GCQ-S, Group Climate Questionnaire, short form. 


\section{Aim 2: To provide Information Regarding Resources Required for EON-Mem}

The major costs and time required for the study are reported in Table 9. On average, sessions lasted approximately one hour, shorter than the anticipated 1.5 hours (see Figure 7 for session-by-session length). Considering both cost for manuals and reimbursement as well as research assistant time, we estimated running one young adult participant in our study cost about $\$ 345$ as itemized below:

- $\quad \$ 152$ for reimbursement and material

- $\quad \$ 31$ for pre-test $(\$ 10 /$ hour*3.1 hours/participant)

- $\quad \$ 31$ for post-test (\$10/hour*3.1 hours/participant)

- $\quad \$ 27$ for research assistants being trained (\$10/hour*(104.5 total hours/39 participants))

- $\quad \$ 17$ for research assistants training another (\$10/hour*(65 total hours/39 participants)

- $\quad \$ 62$ for scoring and data entry (\$10/hour*(241.5 total hours/39 participants))

- $\quad \$ 7$ for recruiting (\$10/hour*(25.3 total hours/39 participants))

- $\quad \$ 6$ for fidelity checks (( $\$ 10 /$ hour*1.0 hour per session*4.6 sessions per group)/7.8 participants per group)

- $\quad \$ 12$ for leading sessions ((\$20/hour*1.0 hour per session*4.6 sessions per group)/7.8 participants per group) 
Table 9

Young Adult: Cost and Time Required for Implementation

\begin{tabular}{|c|c|c|}
\hline \multirow{2}{*}{$\begin{array}{l}\text { Variable } \\
\text { Total cost/participant (US \$) }\end{array}$} & Mean $(S D) /$ Percentage & Range \\
\hline & 345 & \\
\hline $\begin{array}{l}\text { Total reimbursement and materials (per } \\
\text { participant; US \$) }\end{array}$ & $152(41)$ & 66-197 \\
\hline \multirow{2}{*}{$\begin{array}{l}\text { Reimbursement (per participant; US \$) } \\
\text { Protocols and manuals (per participant; } \\
\text { US \$) }\end{array}$} & $87(37)$ & $10-130$ \\
\hline & $65(5)$ & $56-67$ \\
\hline Total research time required (per participant; h) & 18.6 & \\
\hline Pre-testing (per participant; h) & $3.1(0.1)$ & $3.0-3.3$ \\
\hline Post-testing (per participant; h) & $3.1(0.3)$ & $3.0-3.6$ \\
\hline Being trained (per sample; $h$ ) & 104.5 & \\
\hline Training another (per sample; h) & 65.0 & \\
\hline Scoring and data entry (per sample; h) & 241.5 & \\
\hline Recruiting (per sample; $h$ ) & 25.3 & \\
\hline Session time (across sessions; $\mathrm{h}$ ) & $1.0(0.3)$ & $0.5-1.5$ \\
\hline Session two (h) & $0.6(0.1)$ & $0.5-0.8$ \\
\hline Session three $(\mathrm{h})$ & $1.2(0.2)$ & $1.0-1.5$ \\
\hline Session four (h) & $1.1(0.1)$ & $1.0-1.2$ \\
\hline Session five (h) & $1.1(0.1)$ & $0.9-1.2$ \\
\hline Session six (h) & $0.9(0.1)$ & $0.8-1.0$ \\
\hline Session seven $(\mathrm{h})$ & $1.1(0.1)$ & $1.0-1.2$ \\
\hline Session eight $(\mathrm{h})$ & $1.4(0.1)$ & $1.3-1.5$ \\
\hline
\end{tabular}

Next, we estimated running one participant in our study required 18.6 hours of research assistant time as itemized below:

- $\quad 3.1$ hours for pre-test +3.1 hours for post-test

- $\quad 2.7$ hours for research assistant being trained (104.5 hours/39 participants)

- $\quad 1.7$ hours for research assistant training another (65 hours/39 participants) 
- 6.2 hours for scoring and data entry (241.5 hours/39 participants)

- $\quad 0.6$ hours for recruiting (25.3 hours/39 participants)

- $\quad 0.6$ hours for fidelity checks ((1.0 hour per session*4.6 sessions per group)/7.8 participants per group)

- $\quad 0.6$ hours for leading sessions ((1.0 hour per session*4.6 sessions per group)/7.8 participants per group)

Regarding recruitment, we contacted 111 young adults expressing interest in the study (see Figure 1). Seventy-two of the young adults (65\%) met study exclusion criteria. The most common reason participants were excluded was a timing conflict $(n=26)$, followed by no response to the contact $(n=24)$. Other reasons included previous head injury, age, non-native English speaker, no interest, no available slots, or out of town (see Figure 1). Overall, 35\% of the original pool was enrolled in the study, with the most common obstacle to enrollment being a timing conflict. 


\section{Figure 7}

Young Adult: Average Session Length

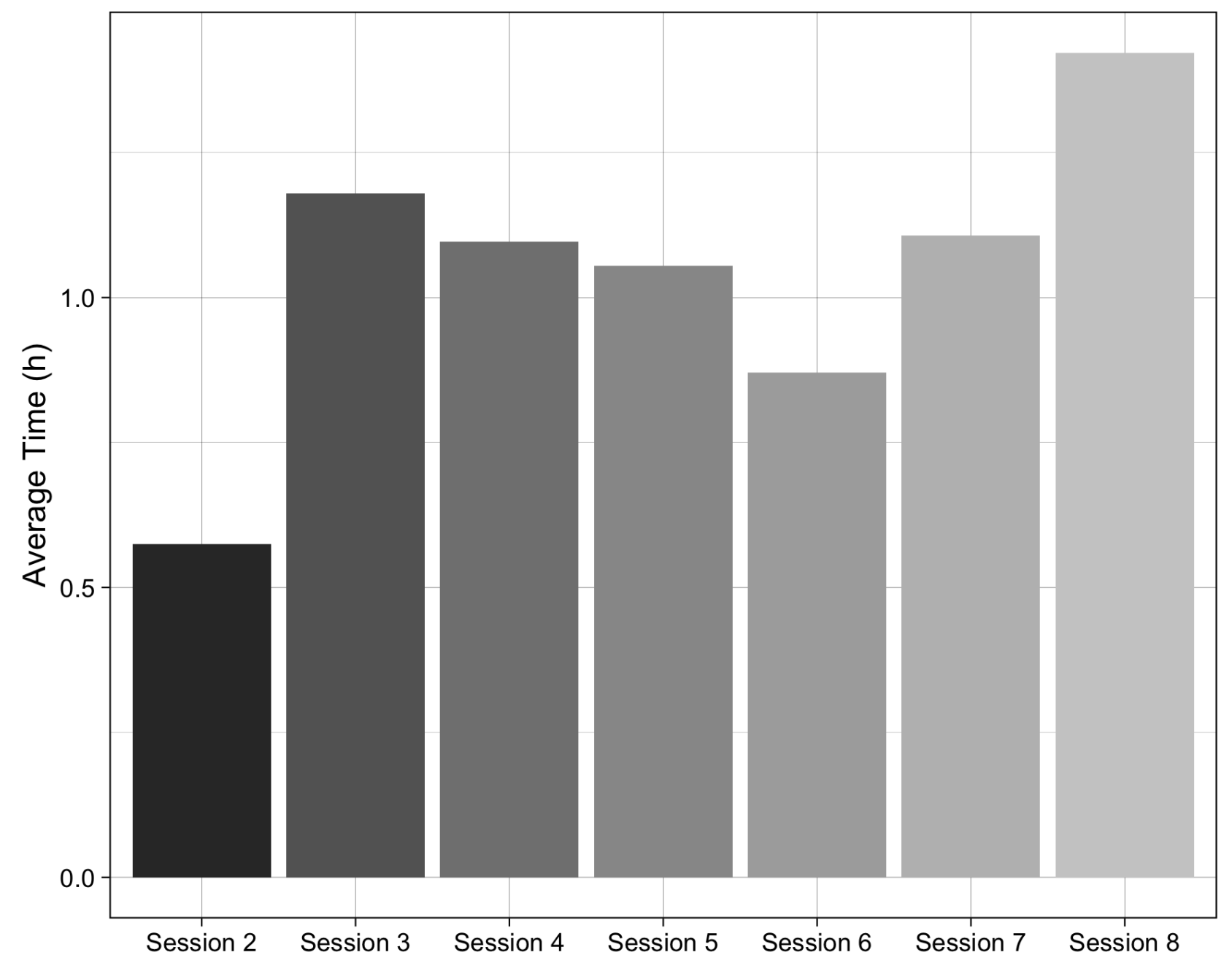

\section{Aim 3: To Assess Change in Memory Performance}

Only six of the 30 young adult participants who received both pre and post-intervention tests $(20 \%$ of the sample) were considered to have improved by the end of the intervention according to the RCI criteria for the RBANS memory index (see Figure 8). Two of the participants scores were reliably worse on the RBANS memory index after the intervention, and the majority of young adults had no reliable change on the RBANS memory index after the intervention (22; Figure 8). On the RBMT, two young adults had reliably better scores, nine of 
the young adults' scores were reliably worse, and 18 young adults had no change after intervention (Figure 8). Regarding Everyday Memory Simulations subtests, we observed the most variability on the Everyday Memory Simulations biographical subtest for face and names (six of the young adults scores were reliably better and 11 young adult scores were reliably worse after intervention). We observed the most improvement on the Everyday Memory Simulations message subtest (four of the young adults scores were reliably better and only one score was reliably worse after treatment). Finally, we observed the most decline in performance on the Everyday Memory Simulations news subtest (none of the young adult scores were reliably better after intervention and four young adult scores were reliably worse). Overall, however, $78 \%$ of participants demonstrated no reliable change across memory indices after intervention (Figure 8). 


\section{Figure 8}

Young Adult: RCI for Memory Indices

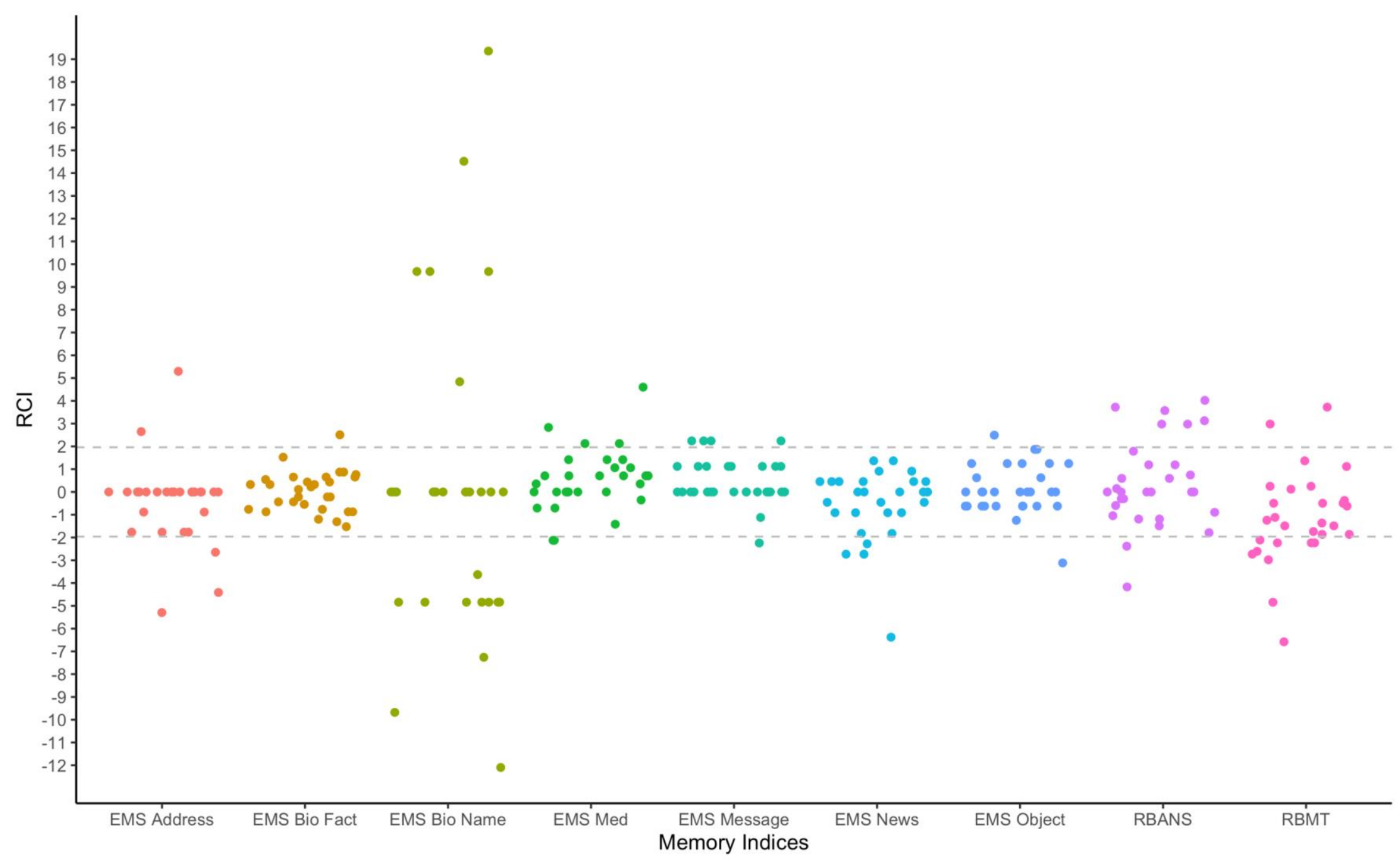


Overall, $85 \%$ of young adults showed no reliable change between pre- and postintervention on the RBANS subtests, although $10 \%$ of participants showed reliable improvement and 5\% of participants showed reliable decline for individual subtests in the RBANS (see Figure 9). Specifically, we observed the most variability on the RBANS story memory immediate recall (four participants performed reliably better and four participants performed reliably worse after intervention). We observed the most improvement on the RBANS picture naming (10 participants performed reliably better and no participants performed reliably worse after treatment). Finally, we observed the most decline in performance on the RBANS semantic fluency (one participant performed reliably better and four participants performed reliably worse after treatment). Overall, most participants (85\%) demonstrated no reliable change on RBANS subtests after intervention (Figure 9). 


\section{Figure 9}

Young Adult: RCI for RBANS Subtests

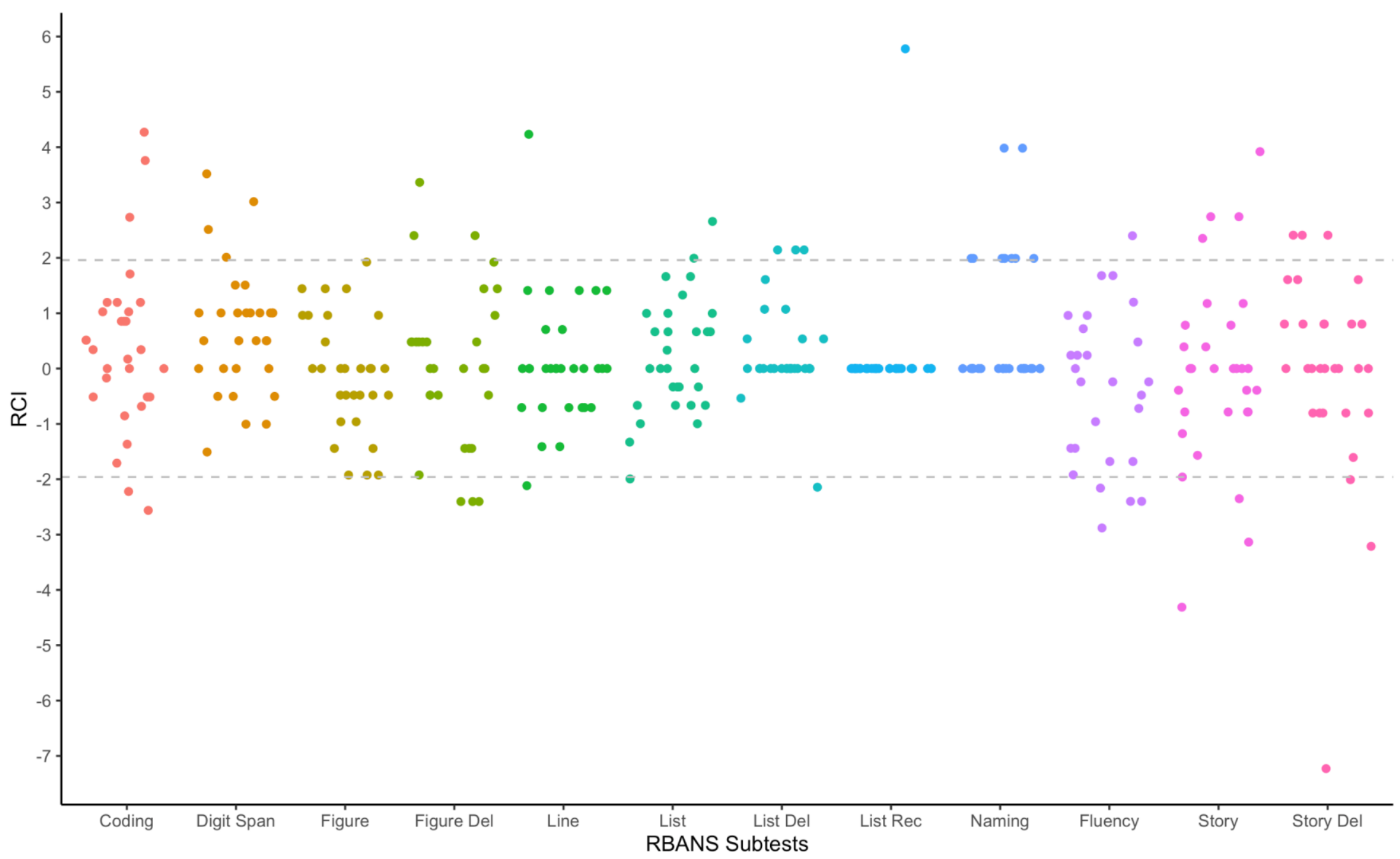




\section{Young Adult: Discussion}

Results from the implementation of EON-Mem with young adults included both positive and unfortunate surprises. On the upside, young adults reported that they enjoyed interacting with others (both other group members and therapists) during treatment. Young adults also reported the presentation was organized and easy to follow; they reported that they particularly enjoyed reviewing homework in class. Regarding expenses, sessions were shorter than expected (usually only one hour despite allotting 1.5 hours for treatment sessions), but cost was quite high (\$345/participant, 18.6 research assistant hours/participant). Data entry (dual coding of all data) especially took a lot of time (1/3 of the total research assistant time). Treatment completion rates and improvement rates were an unfortunate surprise. Only about half (51\%) of the young adults completed the study. Testing attendance was relatively better (77\%), likely due to specific compensation for completing the post-testing session. In our sample, completers compared to non-completers had more males than females, higher intelligence, higher overall cognitive skills (including inhibition skills), lower anxiety and depressive symptoms, higher group cohesion, and higher homework completion.

Regarding improvement rates, only $20 \%$ of the recruited young adult sample improved on the primary variable of interest, the delayed memory index score on the RBANS, between pre- and post-treatment. Seven percent performed reliably worse on the RBANS memory measure after treatment. The majority of young adults (73\%) did not demonstrate reliable change on the RBANS memory index after the intervention. A similar pattern, in which over $50 \%$ of young adults did not demonstrate reliable change, was found across most memory and cognitive measures. In our young adult sample, improvers compared to non-improvers had more females, lower intelligence and cognitive skills, lower inhibition and switching skills, higher group 
cohesion, higher homework completion, and were younger. Finally, young adults completed less than half (47\%) of the homework assignments given in treatment. Overall, although the young adults enjoyed the social interaction and presentation, many of them dropped out of treatment, had poor homework completion, and demonstrated no reliable change after treatment. Further the cost for the study was higher than expected.

One likely explanation for most of the unfortunate surprises (completion, improvement, and homework rates) in the young adult sample was low motivation. In the exit interview, many young adults described feelings of apathy and amotivation toward the treatment. Several young adults made comments about having a good memory and good memory strategies prior to treatment. Further, they found it a stretch to try to apply the treatment within their own lives. Thus, although some may have had an interest in memory skills, it is likely that many in the young adult sample were mainly interested in the study for compensation (SONA/money). In addition, some young adults reported the requirements for participation were too burdensome for the compensation received. This is supported by the fact that eight percent dropped out after preintervention test (at which point they received SONA credit), before even beginning the treatment. A high portion (including many that did not complete treatment) also returned for post-intervention test, at which they were paid a higher amount compared to other sessions $(\$ 20)$. Other barriers reported by the young adults may have also had an impact on attrition rates, including variable/poor timing for the reminders sent and difficult content (e.g., peg words), although participants were not unified in their opinion of session content.

Those who completed treatment in our sample were higher functioning than those who did not complete treatment (e.g., higher intelligence, higher cognition, positive perception of group cohesion, and higher emotional functioning). Perhaps participants who completed 
treatment had sufficient resources (e.g., cognitive ability, emotional functioning, social support) to continue attending treatment, even with relatively low compensation for their time. Next, participants who improved in our sample had a lower cognitive baseline compared to those that did not improve; perhaps the skills taught in EON-Mem were too easy for the majority of young adults in this sample. Finally, both improvers and completers in our group had higher group cohesion, suggesting a potential association between group cohesion and positive outcomes in our young adult sample, as supported in the previous research (Burlingame et al., 2013).

\section{Revisions for Older Adult Sample made in Response to Young Adult Findings}

Based on findings above and our anticipated experiences with older adults, we made several changes to the recruitment, sessions, homework, and data entry. First, due to the poor attendance and the confusion about requirements of participation in the young adult sample, we revised the recruitment script by reducing redundancies. We ended the script with an emphasis on the importance of attending all treatment sessions (see supplementary material). We recruited groups of ten participants instead of eight participants in light of the high dropout rate, even early on in treatment.

Regarding sessions, we encouraged therapists to continue creating a positive environment as felt in the young adult sample, and requested they spend more time on introductions/building rapport in the first session in order to increase cohesion early on. We also asked therapists to explain the flexibility of applying strategies (e.g., using parts of a strategy at one time and finishing other parts at a later time) in efforts to improve applicability. Due to high dropout rates we increased the frequency of compensation: fourth session $(\$ 40)$, sixth session $(\$ 20)$, and ninth session (\$40). In addition, we bought bottles of water to bring to the older adult sessions. We cut down on session content as we anticipated more participation and a slower pace in the older adult 
session (provided a feedback questionnaire but did not explain in depth, cut out a few practice examples-hike, bus stop, did not review accomplishments with partner). We also moved the session times earlier in the day to accommodate winter travel and dinner time for older adults (4:00 PM-5:30 PM, 5:30 PM-7:00 PM).

Due to low homework completion and the number of reported homework barriers we made several changes to the homework. Per participant request, we asked therapists to spend more time helping participants plan and review their homework assignment at the beginning and end of each session in order to increase applicability and reduce barriers. This included helping the participants plan specific material with which to practice at home. We also cut down on the number of homework assignments (removed four) in order to assign only one set of daily homework assignments per session. Finally, due to the confusion expressed in the young adult sample, we asked each older adult group when they would like to receive text reminders and accommodated requests.

Lastly, because one-third of the research assistant time was spent on scoring and data entry, we simplified the data entry process. We entered only numeric data, and not raw, verbal answers as we had done in the young adult sample. We did not continue to spot check raw data, although we did continue with dual entry of scored data. We chose to include the RBANS subtests to the analysis in order to look at different aspects of cognition. We also added an individual exit interview to the post-intervention test as many young adult participants were not in attendance for the group exit interview at the final treatment session. Finally, we made it routine to check all homework assignments in each participant's EON-Mem workbook at the post-intervention test to reduce missing data. After implementing the changes described above, we continued the study with the older adult sample (see below). 


\section{Older Adult: Method}

\section{Older Adult: Participants}

Recruiting. We received written, informed consent from each participant and the study was approved by the Institutional Review Board (IRB). We recruited older adults, as defined in our study as age 65 and older based on definitions in previous studies (Fritsch et al., 2014; Jonker et al., 2000; Smith et al., 2009). Participants were recruited using fliers, word of mouth, posts to the community and at local universities, and mail or email to older adults in the community. We followed the same procedure for phone screening (see Young Adult: Participants) with a modified script incorporating revisions stated above (see revised older adult script on osf.io/xz4ty). Older adults received payments in three increments: $\$ 40$ at session $4, \$ 20$ at session 6, and \$40 upon completion of the post-intervention assessment. Participants who dropped out prior to the post-intervention assessment were offered $\$ 10 /$ session for each session (\$20 for post-test session) attended. We provided water bottles at each session for older adults.

Based on the rationale for sample size and dropout rates given in the young adult method (see Young Adult: Participants) we aimed to include approximately 24 participants (four groups of six participants) in the older adult sample. We planned to recruit 40 participants (four groups of 10 participants) given the higher-than-expected dropout rates in the young adult sample. We planned to terminate data collection when we reached the minimum desired sample size (24 completers); however, due to major limitations imposed on social gatherings during the COVID19 pandemic (particularly in the older adult population), the full dissertation committee approved discontinuing treatment early (see Appendix $\mathrm{C}$ for email record of the committee decision to discontinue the older adult groups due to COVID-19) and we only recruited three groups of older adults (26 recruited: one group of seven, one group of nine, one group of 10; 10 completers). See 
Figure 10 for recruitment flowchart. Specifically, in group two, four participants did not attend session seven due to COVID-19 restrictions and we did not hold session eight due to events related to COVID-19. Similarly, we did not hold sessions seven and eight for group three due to COVID-19 restrictions.

Figure 10

Older Adult: Recruitment Chart

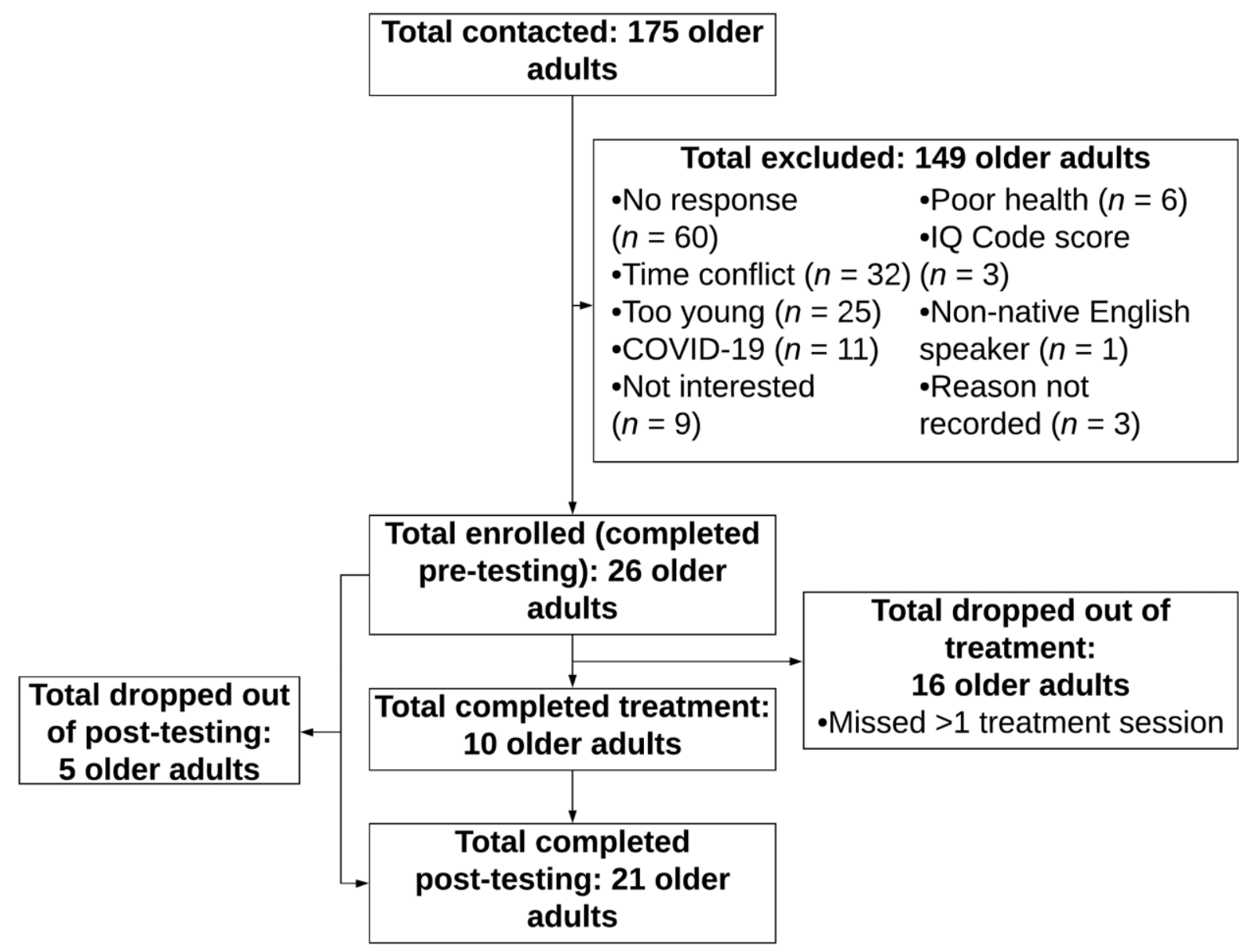

Exclusion criteria. Exclusion criteria for older adults followed those of the large ACTIVE trial, with a few modifications for the current study (Rebok et al., 2014): cognitive dysfunction on the Informant Questionnaire on Cognitive Decline in the Elderly (IQCODE) as evidenced by a score 
of 3.3 or more (Jorm, 1994; Pandharipande et al., 2013); history of moderate-to-severe TBI, stroke, or dementia/neurological diagnoses; current chemotherapy or radiation; brain cancer; poor sensory function including uncorrected vision, hearing, or speaking impairments that could interfere with learning the intervention, including non-native English speakers. The current sample of older adults $(n=26 ; 14$ females) had an average age of 71.6 years $(S D=6.1)$ and averaged 16.8 years $(S D=3.0)$ of education.

\section{Older Adult: Procedures}

Procedures for older adult sample followed that of young adults (see Young Adult: Procedures) with three exceptions. First, as part of the revision process (see revisions in Young Adult: Discussion above), session content was adjusted for older adults (see older adult fidelity

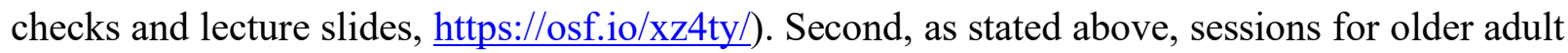
groups two and three were discontinued due to social restrictions enforced during the COVID-19

pandemic. Finally, post-intervention assessments were performed early for older adult group two (at week eight) and older adult group three (at week seven) due to the COVID-19 pandemic.

\section{Older Adult: Measures}

Measures were largely the same between the older adult sample and young adult sample (see Young Adult: Measures, Young Adult: Weekly Measures) with one exception. As part of the revision process for implementation with older adults (see Young Adult: Discussion), we performed individual exit interviews for each participant at the post-intervention assessment in addition to group exit interviews at session eight. Individual exit interviews were included because some participants did not attend the group exit interview and because the group exit interview is limited in time. The internal consistency (Cronbach's alpha) for the delayed portion of each Everyday Memory Simulation subtest administered to the older adult sample was 
acceptable with the exception of one of the biographical information subtests: message delivery 0.81 , address and phone number 0.94 , object location 0.78 , news article 0.85 , medical instructions 0.70 , biographical information face-name pair 0.95 , biographical information facefact pair 0.58 .

\section{Older Adult: Analyses}

All analyses performed for older adults were the same as analyses performed for young adults (see Young Adult: Analyses). All data were dually entered to limit data entry errors. As with the younger adults, outliers were broadly defined in our study as data points three interquartile range (IQR) beyond the median or outstanding data points as determined by visual inspection of boxplots (data points located outside of the whiskers of the boxplots; total data point outliers $=122$ ). Twenty-six of the data points were corrected due to data input/scoring errors. Data entry, scoring method, and standard administration were found appropriate for all other outliers, and thus the remaining 96 outliers were left in their original state. Twenty percent of the data was considered missing due to high treatment dropout rates.

See Table 10 for reliability coefficients used in calculating RCI from the older adult sample. The sensitivity analysis for Everyday Memory Simulations RCI revealed similar results for older adults as found for young adults. The number of improvers was not much different depending on the reliability coefficient used (using the Everyday Memory Simulations internal reliability coefficient: $14 \%$ improved, $74 \%$ did not change, $12 \%$ performed worse; using the RBMT reliability coefficient: $15 \%$ improved, $73 \%$ did not change, $12 \%$ performed reliably worse), providing some support for the assumption made in calculating RCI for Everyday Memory Simulations. Finally, for deviations made in the current study from the original preregistration/prospectus proposal, see Table 11. 


\section{Table 10}

Older Adult: Reliabilities for Calculating Reliable Change Indices

\begin{tabular}{|c|c|c|}
\hline Test & Source of Reliability & Coefficient \\
\hline Delayed Address and Phone Number Total Points & Current sample: Cronbach's alpha & 0.94 \\
\hline Delayed Object Locations Total Points & Current sample: Cronbach's alpha & 0.78 \\
\hline Delayed Medical Instructions Total Points & Current sample: Cronbach's alpha & 0.70 \\
\hline Delayed Newspaper Article Total Points & Current sample: Cronbach's alpha & 0.85 \\
\hline Bio: Sum of Delayed Faces and Names Total Points & Current sample: Cronbach's alpha & 0.95 \\
\hline Bio: Sum of Delayed Facts Total Points & Current sample: Cronbach's alpha & 0.58 \\
\hline Message Delivery Content Score & Current sample: Cronbach's alpha & 0.81 \\
\hline RBANS delayed memory & Manual: Test-retest coefficient & 0.80 \\
\hline Rivermead General Memory Index & Manual: Reliability coefficient & 0.87 \\
\hline RBANS List Learning & Manual: Test-retest coefficient & 0.52 \\
\hline RBANS Story Memory & Manual: Test-retest coefficient & 0.80 \\
\hline RBANS Figure Copy & Manual: Test-retest coefficient & 0.54 \\
\hline RBANS Line Orientation & Manual: Test-retest coefficient & 0.49 \\
\hline RBANS Picture Naming & Manual: Test-retest coefficient & 0.50 \\
\hline RBANS Semantic Fluency & Manual: Test-retest coefficient & 0.52 \\
\hline RBANS Digit Span & Manual: Test-retest coefficient & 0.63 \\
\hline RBANS Coding & Manual: Test-retest coefficient & 0.83 \\
\hline RBANS List Delay & Manual: Test-retest coefficient & 0.60 \\
\hline RBANS List Recognition & Manual: Test-retest coefficient & 0.27 \\
\hline RBANS Story Memory Delay & Manual: Test-retest coefficient & 0.72 \\
\hline RBANS Figure Delay & Manual: Test-retest coefficient & 0.55 \\
\hline
\end{tabular}




\section{Table 11}

Deviations from Pre-registration/Prospectus

\begin{tabular}{|c|c|c|}
\hline Original Plan & Current Study & Reason for Adjustment \\
\hline $\begin{array}{l}\text { Include four YA groups; total of } 24 \\
\text { completers }\end{array}$ & $\begin{array}{l}\text { Included five YA groups ( } 20 \\
\text { completers) }\end{array}$ & $\begin{array}{l}\text { Higher than expected dropout rates } \\
\text { in YA }\end{array}$ \\
\hline $\begin{array}{l}\text { Include four OA groups; total of } 24 \\
\text { completers }\end{array}$ & $\begin{array}{l}\text { Included three OA groups (10 } \\
\text { completers); ended treatment } \\
\text { sessions early; performed } \\
\text { assessments early }\end{array}$ & $\begin{array}{l}\text { COVID-19 restrictions on social } \\
\text { gatherings }\end{array}$ \\
\hline $\begin{array}{l}\text { Pay YA \$100 regardless of SONA } \\
\text { credit }\end{array}$ & $\begin{array}{l}\text { YA received } \$ 130 \text { or } \$ 100 \text { plus } 18 \\
\text { SONA credits }\end{array}$ & $\begin{array}{l}\text { Original groups were paid up to } \\
\$ 130 \text { based on original researcher's } \\
\text { design and recruitment bled into } \\
\text { later groups }\end{array}$ \\
\hline $\begin{array}{l}\text { Include the Routes subtest in } \\
\text { Everyday Memory Simulations }\end{array}$ & Did not include Routes subtests & $\begin{array}{l}\text { Original authors of Everyday } \\
\text { Memory Simulations did not } \\
\text { provide material needed for Routes }\end{array}$ \\
\hline Score GRQ-E according to manual & $\begin{array}{l}\text { Recoded/rescored GRQ-E from a 7- } \\
\text { pt to a 5-pt Likert scale for the YA } \\
\text { group }\end{array}$ & $\begin{array}{l}\text { Used incorrect answering options on } \\
\text { the GRQ-E with YA groups }\end{array}$ \\
\hline
\end{tabular}

Note. YA, young adult sample; OA, older adult sample; GRQ-E, Group Readiness Questionnaire, Expectancy subscale. 


\section{Older Adult: Results}

\section{Aim 1: To Provide Descriptive Statistics about Adherence to Treatment, Cognitive and Emotional Functioning, Participant Feedback, and Relevant Demographic Information}

For information regarding treatment attendance and homework completion for the sample as a whole, see Table 12. Overall, when considering COVID-19 restrictions, older adults attended $85 \%$ of all possible treatment sessions; however, in applying the study's original definition of completion, only 10 of the 26 participants (38\%) successfully completed treatment (attended at least six of the seven sessions). Participants averaged $5.2(S D=1.6)$ of seven possible treatment sessions (74\% of all treatment sessions; see Figure 11$)$. The low treatment completion rates were affected by COVID-19 as pre-COVID-19 treatment attendance was higher than the overall average attendance reported above. Treatment attendance, the number of sessions possible to attend pre-COVID-19, varied by the three older adult groups. Group one was unaffected by COVID-19 and averaged $6.3(S D=1.3)$ of 7 possible treatment sessions $(90 \%)$; group two averaged $4.0(S D=1.8)$ of six possible treatment sessions $(67 \%)$; group three averaged $4.6(S D=0.7)$ of five possible treatment sessions $(91 \%)$. Attendance was $85 \%$ when averaging each participant attendance rate for all possible treatment sessions (prior to COVID-19 changes in policy). Further, up to the point of COVID-related policy change, $58 \%$ of participants had perfect attendance, and 19\% had only missed one session. Interestingly, no participants attended only one or only two of the possible seven sessions. Twenty-one of the 26 participants $(81 \%)$ attended the post-intervention assessment (see Figure 11). Of note, 11 of the 16 participants (69\%) who did not complete treatment returned for post-intervention testing. In sum, treatment attendance was lower than expected, likely due to COVID-19. 


\section{Table 12}

Older Adult: Description of Attendance and Homework for the Sample

\begin{tabular}{|c|c|c|c|}
\hline Variable & $\mathrm{n}$ & Mean (SD)/Percentage & Range \\
\hline Treatment completion category (6+ sessions) & 26 & & \\
\hline Completed & 10 & 38.5 & \\
\hline Dropped out & 16 & 61.5 & \\
\hline Treatment attendance (sessions) & 26 & $5.2(1.6)$ & $0-7$ \\
\hline 0 & 1 & 3.9 & \\
\hline 1 & 0 & 0 & \\
\hline 2 & 0 & 0 & \\
\hline 3 & 2 & 7.7 & \\
\hline 4 & 4 & 15.4 & \\
\hline 5 & 9 & 34.6 & \\
\hline 6 & 3 & 11.5 & \\
\hline 7 & 7 & 26.9 & \\
\hline Testing attendance (sessions) & 26 & $1.8(0.4)$ & $1-2$ \\
\hline 1 & 5 & 19.2 & \\
\hline 2 & 21 & 80.8 & \\
\hline Homework assignments (number completed) & 25 & $30.6(14.1)$ & $0-49$ \\
\hline $0-7$ & 3 & 12 & \\
\hline $8-14$ & 1 & 4 & \\
\hline $15-21$ & 3 & 12 & \\
\hline $22-28$ & 2 & 8 & \\
\hline $29-35$ & 7 & 28 & \\
\hline $36-42$ & 4 & 16 & \\
\hline $43-49$ & 5 & 20 & \\
\hline Days worked on homework (days) & 25 & $30.1(14.3)$ & $0-49$ \\
\hline $0-7$ & 3 & 12 & \\
\hline $8-14$ & 1 & 4 & \\
\hline $15-21$ & 3 & 12 & \\
\hline $22-28$ & 2 & 8 & \\
\hline $29-35$ & 7 & 28 & \\
\hline $36-42$ & 4 & 16 & \\
\hline $43-49$ & 5 & 20 & \\
\hline \multicolumn{4}{|l|}{ Improved category } \\
\hline Improved (RCI 1.96+) & 4 & $20 \%$ & \\
\hline Did not improve $(\mathrm{RCI}<1.96)$ & 16 & $80 \%$ & \\
\hline
\end{tabular}

Note. RCI, reliable change indices. 


\section{Figure 11}

Older Adult: Session Attendance

A Treatment Session Attendance

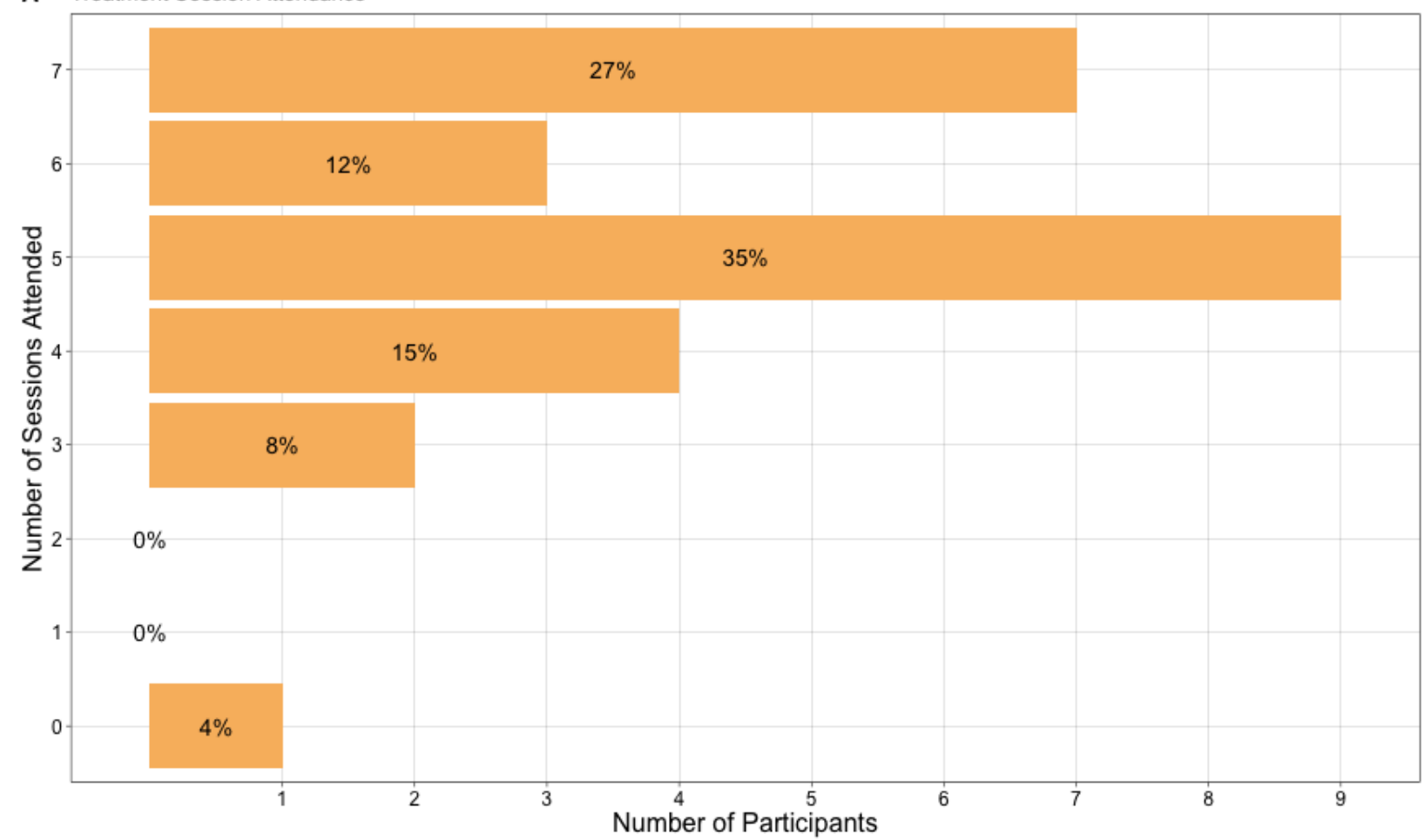

B Testing Session Attendance

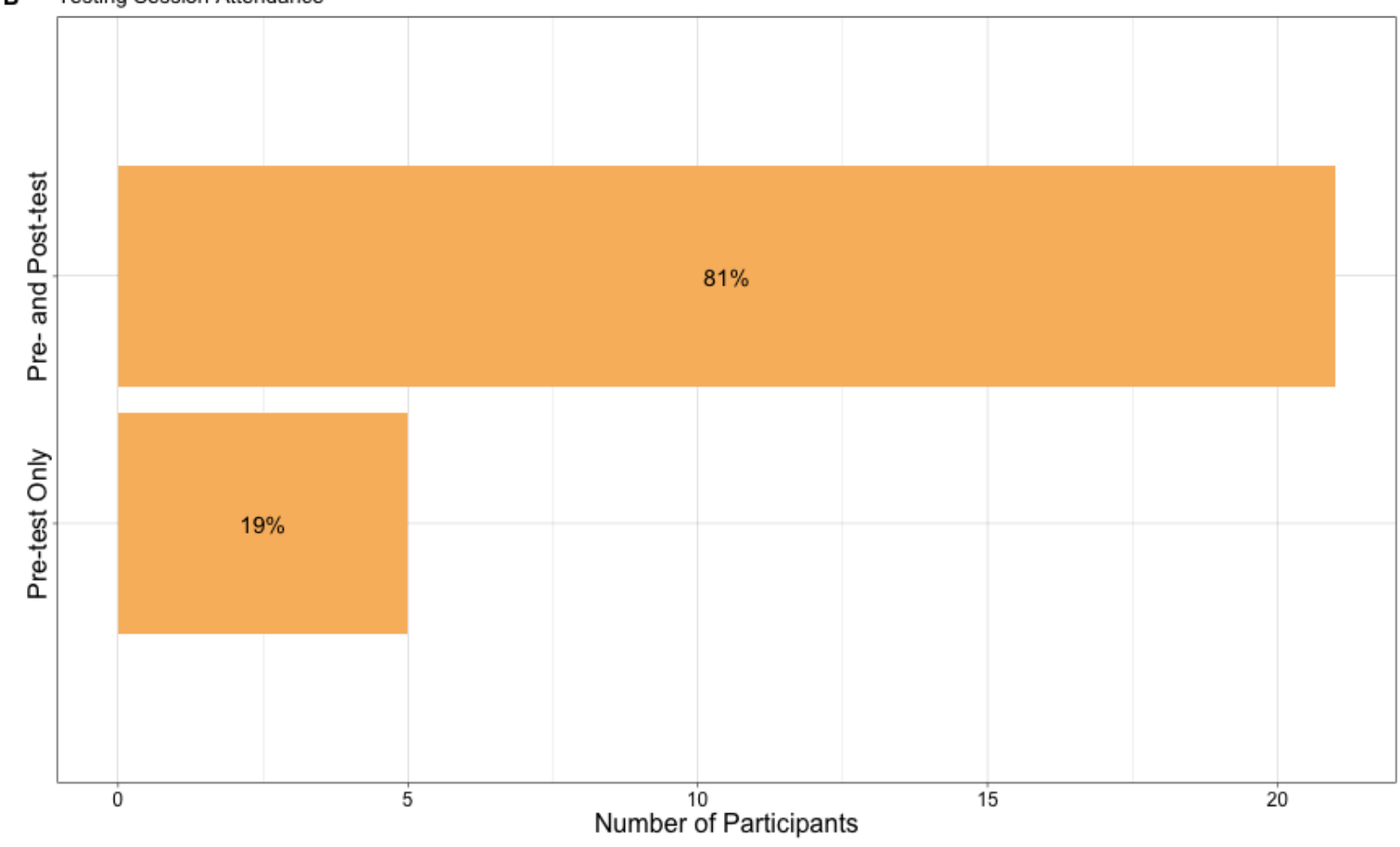


Regarding homework, older adult participants on average completed $30.6(S D=14.1)$ of the 49 possible homework assignments (62\% of all homework assigned) and worked on homework an average of $30.1(S D=14.3)$ of the possible 49 days in treatment $(61.4 \%$; see Figure 12). Although pre-COVID-19 homework completion rates were somewhat higher than overall sample rates given above, the homework completion rates were not nearly as affected by COVID-19 as treatment attendance rates. Group one (unaffected by COVID-19 policy) averaged $37.3(S D=12.8)$ of 49 assignments $(76 \%)$ and worked on homework an average of $37.1(S D=$ $13.0)$ of 49 possible days $(76 \%)$. Group two averaged $21.1(S D=13.3)$ of 42 possible assignments $(50 \%)$ and worked on homework an average of $20.3(S D=12.5)$ of 42 possible days $(48 \%)$; group three averaged $24.6(S D=9.9)$ of 35 possible assignments $(70 \%)$ and worked on homework an average of $24.0(S D=10.3)$ of 35 possible days $(69 \%)$. Homework completion rates were $72 \%$ and participants worked on homework $71 \%$ of the days when averaging each participant homework completion rate prior to COVID-19 related changes in policy. The standard deviations observed in all homework calculations suggests high variability in homework completion among participants. Overall, participants had somewhat low homework completion, even considering COVID-19 as a potential confound. 


\section{Figure 12}

Older Adult: Homework Completion

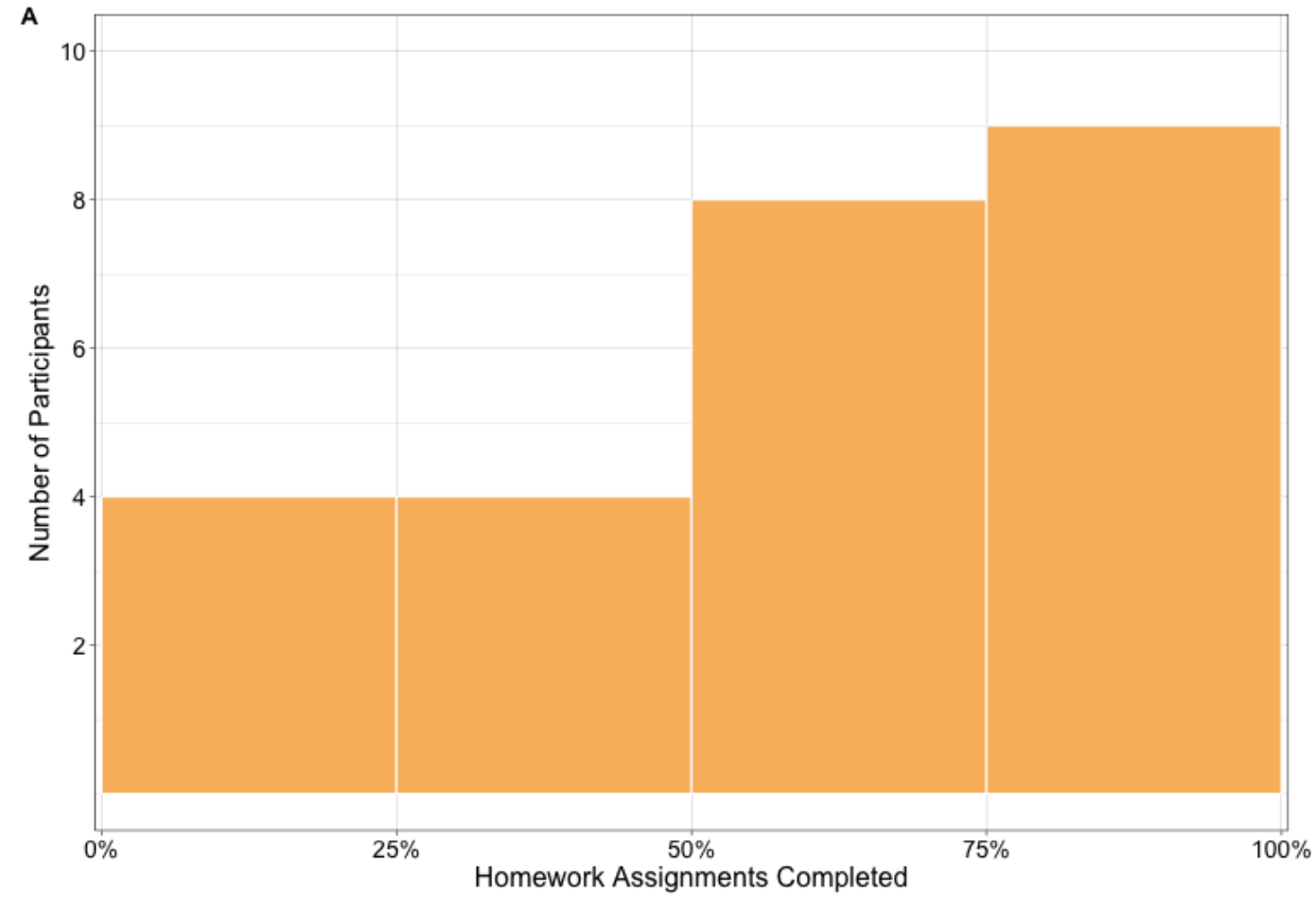

B Time Spent on Homework

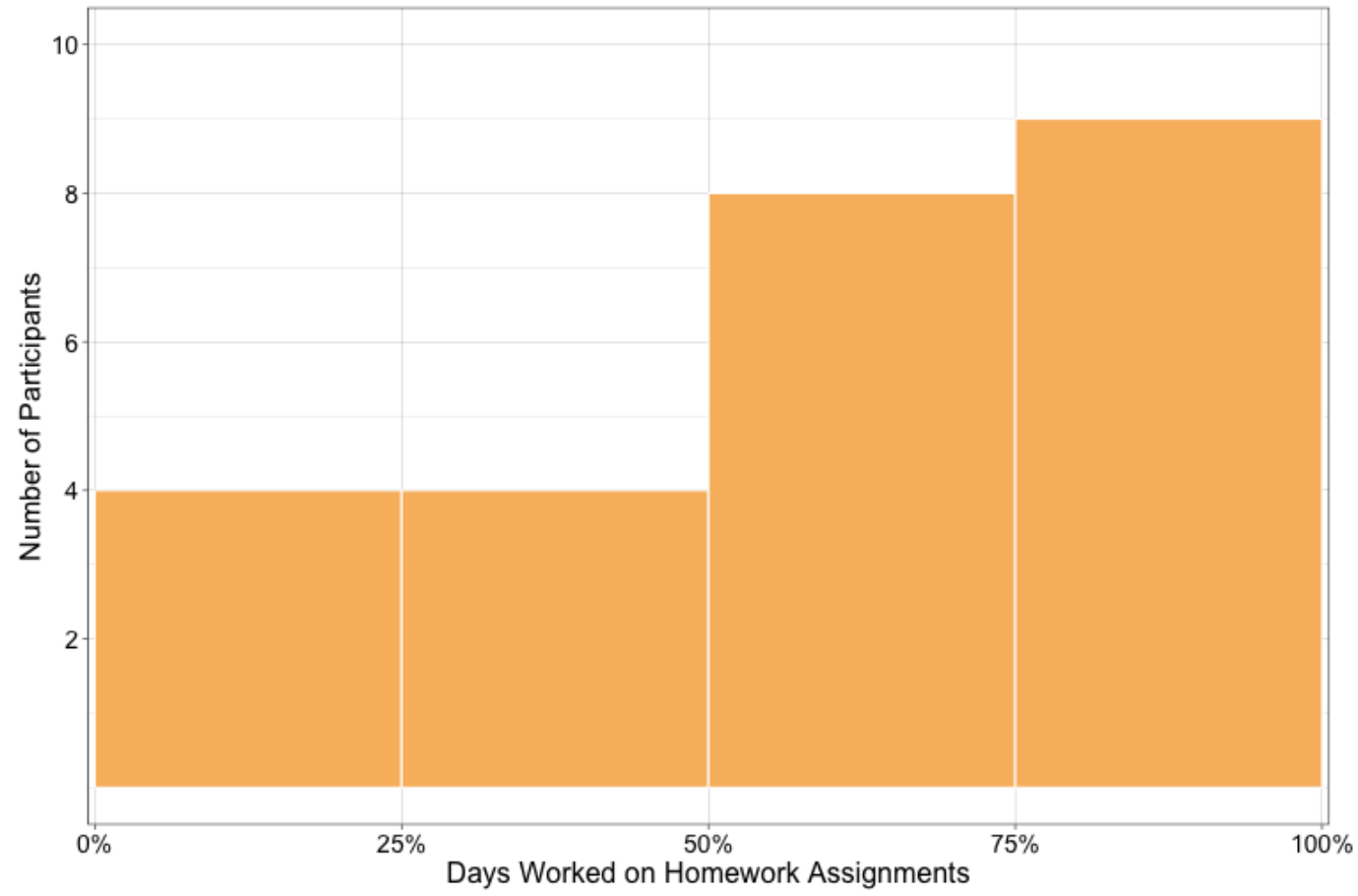


For tables regarding general quantitative and qualitative participant-reported facilitators and barriers to treatment, see Tables 13-14. For more detailed session by session feedback from older adult participants, see Supplemental Tables 3-4 on OSF (ㅇf.io/xz4ty). Supplemental Table 3 includes facilitators and barriers described by participants on their weekly Participant Feedback Form for each of the seven treatment sessions. Supplemental Table 4 includes average scores for each question on the Participant Feedback Form and for each question on the GCQ-S Engagement subscale for each of the seven treatment sessions. 
Table 13

Older Adult: Quantitative Participant Feedback from Weekly Treatment Session Questionnaires

\begin{tabular}{lcc}
\hline Variable & Mean $(S D) /$ Percentage & Range \\
\hline GCQ-S Engagement subscale: Total & $3.8(0.9)$ & $2.0-5.7$ \\
\hline Question 1: liked each other. & $4.5(0.8)$ & $3.0-6.0$ \\
Question 2: tried to understand. & $4.5(0.8)$ & $2.5-6.0$ \\
Question 3: sense of participation. & $4.5(0.8)$ & $2.8-6.0$ \\
Question 4: confront each other. & $3.1(1.5)$ & $0.0-6.0$ \\
Question 5: reveal sensitive info. & $2.4(1.4)$ & $0.6-5.0$
\end{tabular}

Participant Feedback Form

Question 1: training useful.

$1.5(0.4)$

$1.0-2.3$

Question 2: education clear.

$1.4(0.4)$

$1.0-2.2$

Question 3: presentation helpful.

$1.4(0.4)$

$1.0-2.4$

Question 4: group practice helpful.

$1.5(0.4)$

$1.0-2.7$

Question 5: therapist clear.

$1.2(0.3)$

$1.0-2.0$

Question 6: homework useful.

$1.8(0.5)$

$1.0-3.7$

Question 7: no barriers to homework.

$2.0(0.8)$

$1.0-4.0$

Question 8: training helped in life.

$2.0(0.5)$

$1.0-3.0$

Note. GCQ-S, Group Climate Questionnaire, short form.

\begin{tabular}{r}
\hline GCQ-S Key \\
Not at all: 0 \\
A little bit: 1 \\
Somewhat: 2 \\
Moderately: 3 \\
Quite a bit: 4 \\
A great deal: 5 \\
Extremely: 6
\end{tabular}

Participant Feedback Form Key

Strongly agree: 1 Somewhat agree: 2 Do not agree/disagree: 3

Somewhat disagree: 4 Strongly disagree: 5 
Table 14

Older Adult: Reported Facilitators and Barriers to EON-Mem Treatment

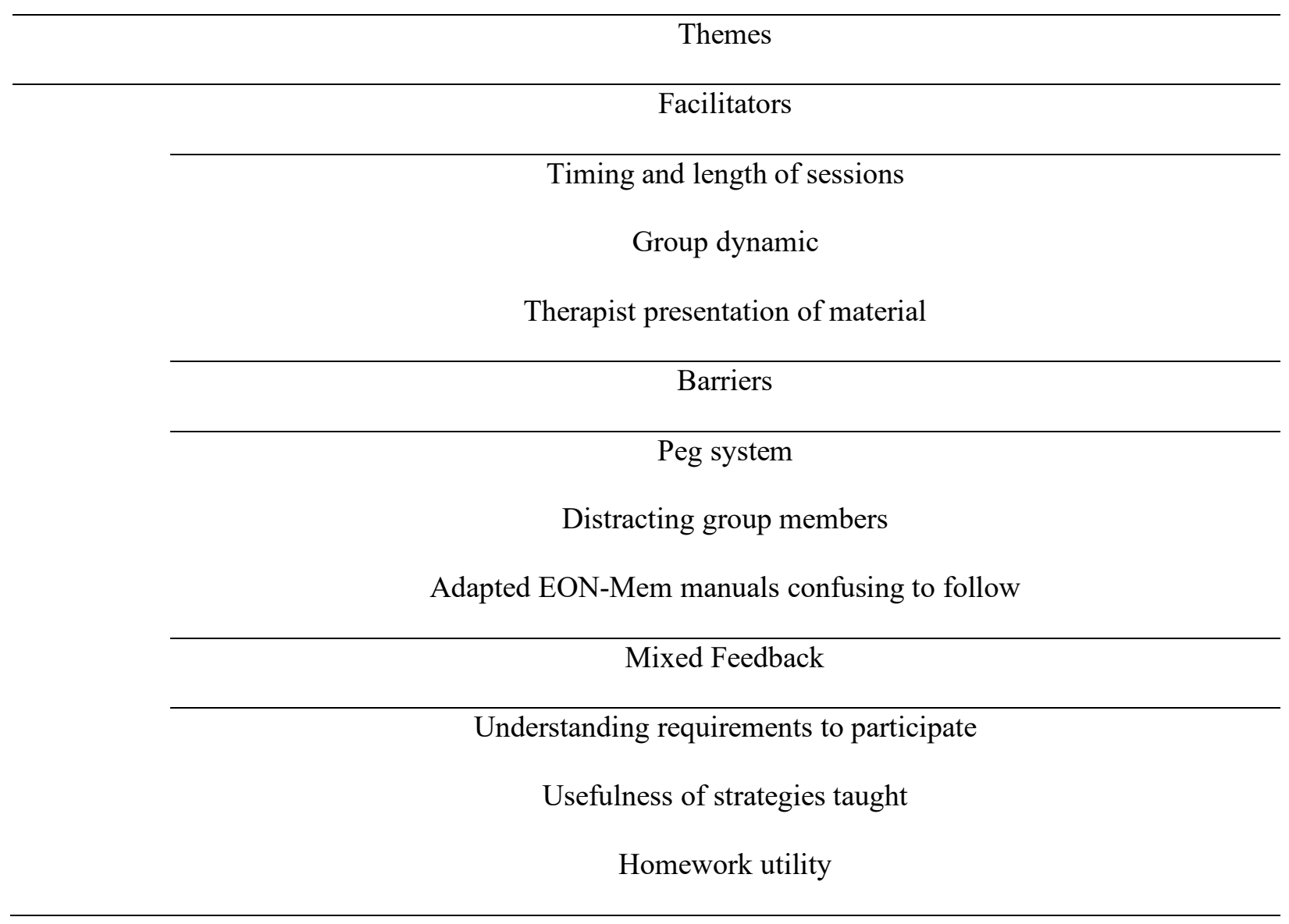

Older adults reported several facilitators in treatment. First, many participants reported no difficulties with scheduling treatment or testing sessions or with session length (30 related comments). Next, the older adults enjoyed the group dynamic. Participants commented on an appropriate group size, positive friendships and support, and the benefits of learning from other members in the group (50 related comments). Participants generally liked other group members as evidenced by ratings on question one of the GCQ-S Engagement subscale (see Table 13 and Figure 13), finding other members as "open," "fun," and "friendly." Of note, a few members felt they had personality differences with certain group members and some wished for a bigger group (as noted in the one group of six; 10 related comments). Finally, participants gave positive 
feedback regarding therapist presentation of EON-Mem, as evidenced by responses to questions one and five on the Participant Feedback Form (see Table 13 and Figure 14). Most participants found the presentation was clear and enjoyed working with the therapist (51 related comments). Of note, a minority of participants found the presentation was confusing (e.g., material covered too quickly, disorganized, inconsistent) and wished the therapists were more energetic and engaging (15 comments). 
Figure 13

Older Adult: GCQ-S Engagement Subscale

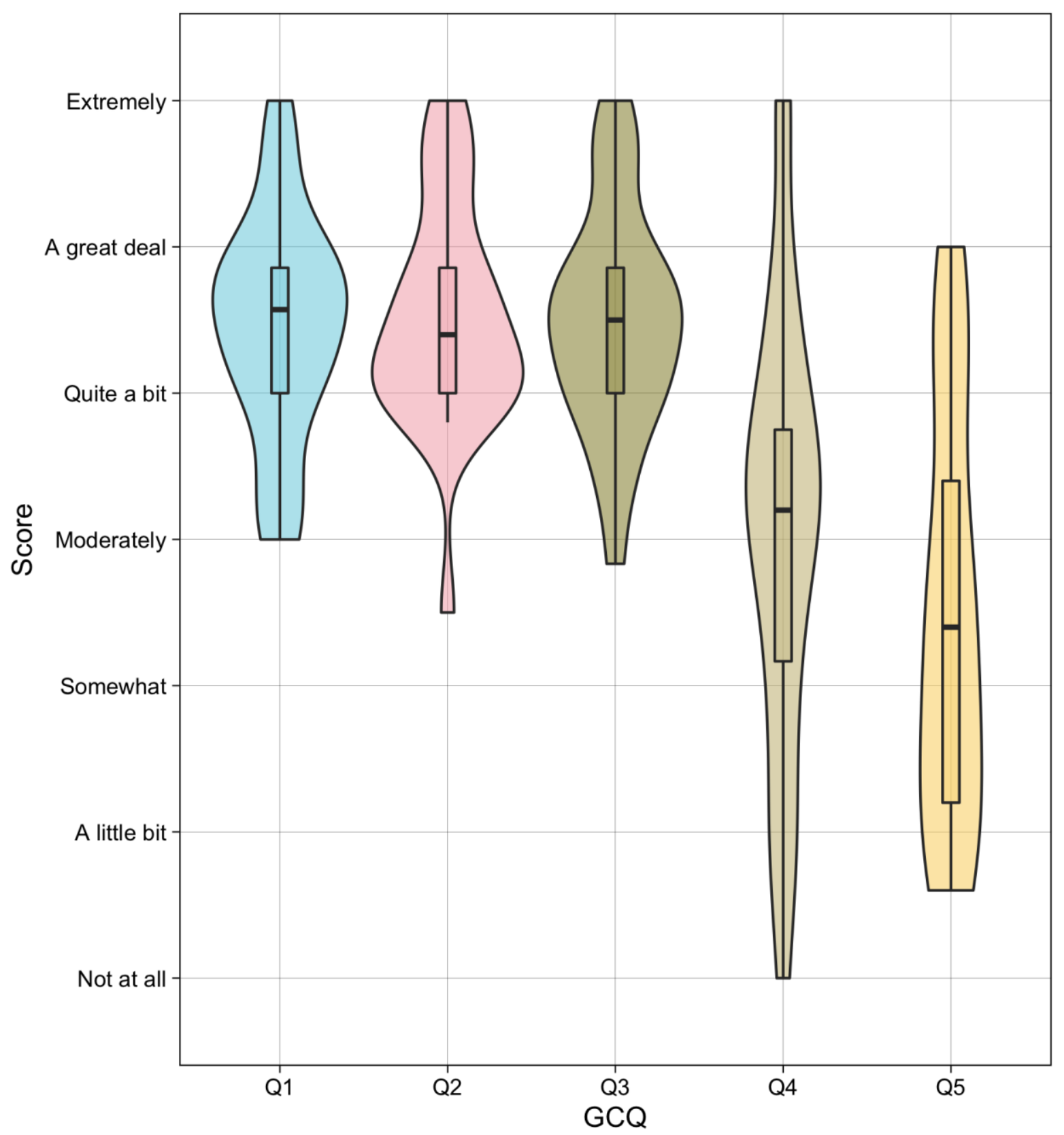

Note. GCQ-S: Group Climate Questionnaire, short form

Q1: The members liked and cared about each other.

Q2: The members tried to understand why they do the things they do, tried to reason it out.

Q3: The members felt what was happening was important and there was a sense of participation.

Q4: The members challenged and confronted each other in their efforts to sort things out.

Q5: The members revealed sensitive personal information or feelings. 


\section{Figure 14}

Older Adult: Participant Feedback Form

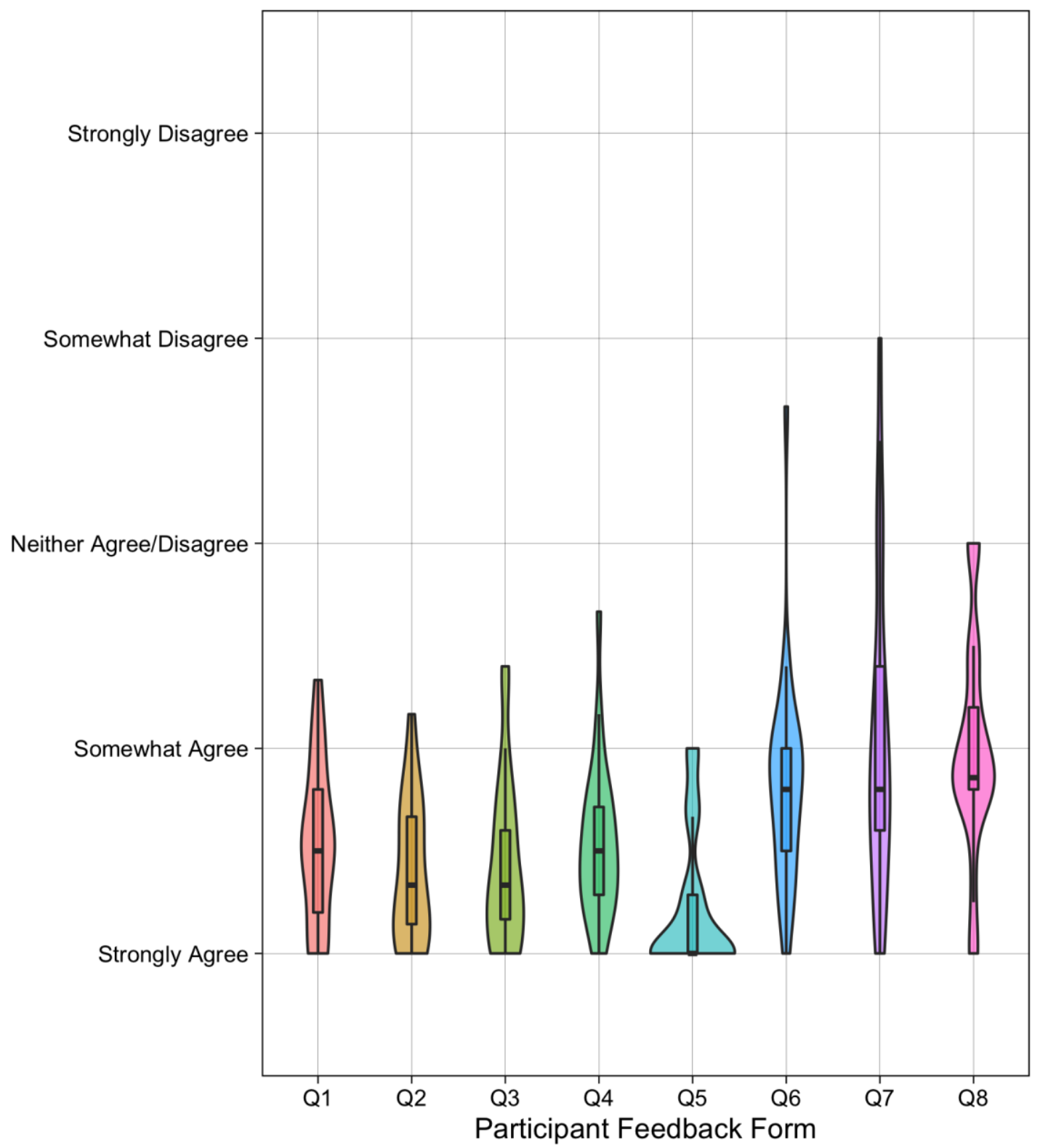

Note. Q1: The training was useful.

Q2: The educational portion was clear.

Q3: I found the presentation helpful.

Q4: The in-group practices were helpful.

Q5: The therapist was clear and easy to follow.

Q6: The homework was useful.

Q7: I did not encounter barriers to completing the homework.

Q8: The training helped me in everyday life. 
The older adults also described three major barriers to treatment (Table 14). First, participants consistently disliked using the peg system (used to memorize numbers; 15 comments). Many felt the peg system was too much work, unrelatable, and unnecessary as they already had a system for remembering numbers. However, a few participants enjoyed peg words and liked making visual stories with the numeric information. Second, several older adults felt the group, and particular individuals in the group, were distracting to their learning (e.g., “chatty;" 12 related comments). Of note, comments about chatty group members happened in particular sessions with particular groups; often, only one older adult was distracting or only a couple of members were distracted by other members. Third, and finally, some members took issue with how EON-Mem material was used (12 comments). For example, some members wished to review certain PowerPoint slides or therapist manuals at home, but could not due to copyright. Additionally, because we only used portions of the full EON-Mem manual some members were confused about which homework to complete between sessions.

Participants reported mixed opinions regarding some topics. First, some felt they understood the requirements to participate prior to participating (four comments), whereas other wanted more detail before committing to sessions (four comments; e.g., syllabus of content to be covered, purpose of study, homework expectations, length of time). Participants also varied in which sessions they found most helpful. Some generally appreciated the strategies (eight comments; evidenced by questions one and three on the Participant Feedback Form, see Table 13 and Figure 14); some liked strategies taught for specific domains (ten comments): names/faces, numbers, oral and written information. Others did not find benefit of the strategies as supported by responses to question eight on the Participant Feedback Form (see Table 13 and Figure 14). For example, participants felt the strategies required too much work and were not compatible 
with already learned strategies (25 comments, 15 of which were related to peg words). Many participants did not like peg words, a few did not need help with object locations (four comments), and a one did not like oral and written information. Finally, participants similarly varied in their views of homework utility. Some participants liked homework and found assignments useful and enjoyable (13 comments; Participant Feedback Form, question six, see Table 13 and Figure 14), whereas others described them as "easy," "boring," "redundant," and "busy work," (12 comments) and a third group described them as "overwhelming" and "difficult" and "of questionable value" (21 comments; see Participant Feedback Form, question seven, Table 13). The view of the homework seemed to depend on the specific participant and specific assignment, which varied quite a bit between participants (see distribution of questions six and seven on the Participant Feedback Form). Overall, participant opinions about strategies and homework was extremely heterogenous and dependent on the individual.

Cognitive and emotional functioning, demographic factors, feedback, and fidelity to treatment for various groups within the older adult sample are given in Tables 15-17. Within the following section, we will use comparative words such as "differences," "than," "higher," and "lower" to describe the data. Unlike in inferential data, differences and trends described do not implicate statistically significant differences or predictions, they are simply descriptors for understanding the data. All differences in pre-treatment variables between groups can be observed in Tables 15-17. Below, we note variables in which group effect sizes are 0.5 or greater within our sample. In our sample, none of the variables had an effect size of 0.5 or greater on treatment completion groups, except of course higher homework completion rates. In our sample, improvers compared to non-improvers had higher predicted intelligence, lower cognitive skills, lower inhibition skills, higher depression, and higher homework and session completion. 
None of the variables demonstrated an effect size of 0.5 or greater on homework completion groups, except of course the amount of homework completed and sessions attended by participants. 


\section{Table 15}

Older Adult: Baseline Information for Completers in the Older Adult Sample

\begin{tabular}{lllll}
\hline Variable & $\begin{array}{l}\text { Completed } \\
(n=10)\end{array}$ & $\begin{array}{l}\text { Dropped out } \\
(n=16)\end{array}$ & Cohen's $d$ & $95 \%$ Confidence Interval \\
\hline Age & $71.3(6.0)$ & $71.8(6.4)$ & 0.1 & {$[-0.7,0.9]$} \\
Gender (\% female) & $50 \%$ & $56 \%$ & -0.1 & {$[-0.9,0.7]$} \\
Education & $16.9(3.6)$ & $16.7(2.6)$ & -0.1 & {$[-0.9,0.7]$} \\
TOPF & $116.0(4.8)$ & $114.6(9.0)$ & -0.2 & {$[-1.0,0.6]$} \\
RBANS A total index & $111.4(14.7)$ & $114.9(13.5)$ & 0.3 & {$[-0.6,1.1]$} \\
DKEFS inhibition & $9.8(2.3)$ & $10.4(3.4)$ & 0.2 & {$[-0.6,1.0]$} \\
DKEFS inhibition/switching & $10.4(2.6)$ & $10.6(3.2)$ & 0.1 & {$[-0.8,0.9]$} \\
DKEFS sorting & $13.4(2.8)$ & $13.3(3.2)$ & 0.0 & {$[-0.8,0.8]$} \\
HADS anxiety & $5.3(2.8)$ & $5.4(4.2)$ & 0.0 & {$[-0.8,0.8]$} \\
HADS depression & $3.8(2.7)$ & $2.8(2.2)$ & -0.4 & {$[-1.2,0.4]$} \\
GRQ-E & $7.3(2.2)$ & $7.4(3.0)$ & 0.0 & {$[-0.8,0.8]$} \\
GCQ-S Engagement subscale & $3.8(0.9)$ & $3.8(1.0)$ & 0.0 & {$[-0.8,0.8]$} \\
HW assignments & $40.8(6.0)$ & $23.8(14.0)$ & -1.5 & {$[-2.4,-0.6]$} \\
Days worked on HW & $40.3(7.0)$ & $23.3(14.0)$ & -1.5 & {$[-2.3,-0.5]$} \\
Session attendance & $6.7(0.5)$ & $4.2(1.3)$ & -2.3 & {$[-3.3,-1.3]$} \\
\hline
\end{tabular}

Note. HW, homework; TOPF, Advanced Clinical Solutions: Test of Premorbid Functioning; RBANS A, Repeatable Battery for Neuropsychological Status Update A; DKEFS, Delis-Kaplan Executive Function System; HADS, Hospital Anxiety and Depression Scale; GRQ, Group Readiness Questionnaire, Expectancy subscale; GCQ-S, Group Climate Questionnaire, short form. 


\section{Table 16}

Older Adult: Baseline Information for Improvers in the Older Adult Sample

\begin{tabular}{lllll}
\hline Variable & $\begin{array}{l}\text { Improved } \\
(n=4)\end{array}$ & $\begin{array}{l}\text { Did not improve } \\
(n=16)\end{array}$ & Cohen's $d$ & $\begin{array}{l}95 \% \text { Confidence } \\
\text { Interval }\end{array}$ \\
\hline Age & $71.5(5.5)$ & $70.1(5.8)$ & -0.3 & {$[-1.3,0.9]$} \\
Gender (\% female) & $50 \%$ & $50 \%$ & 0.0 & {$[-1.1,1.1]$} \\
Education & $18.3(3.7)$ & $16.9(3.0)$ & -0.4 & {$[-1.5,0.7]$} \\
TOPF & $118.8(3.3)$ & $114.8(7.6)$ & -0.6 & {$[-1.7,0.5]$} \\
RBANS A total index & $106.8(11.2)$ & $114.3(13.6)$ & 0.6 & {$[-0.6,1.7]$} \\
DKEFS inhibition & $9.3(1.5)$ & $10.9(2.5)$ & 0.7 & {$[-0.5,1.8]$} \\
DKEFS inhibition/switching & $10.8(1.0)$ & $10.7(2.6)$ & 0.0 & {$[-1.1,1.1]$} \\
DKEFS sorting & $12.8(1.5)$ & $13.8(3.6)$ & 0.3 & {$[-0.8,1.4]$} \\
HADS anxiety & $6.3(4.6)$ & $5.0(3.9)$ & -0.3 & {$[-1.4,0.8]$} \\
HADS depression & $5.8(2.8)$ & $2.6(2.3)$ & -1.3 & {$[-2.5,-0.1]$} \\
GRQ-E & $6.0(2.9)$ & $6.9(2.5)$ & 0.3 & {$[-0.8,1.4]$} \\
GCQ-S Engagement subscale & $3.9(0.9)$ & $3.8(1.0)$ & -0.1 & {$[-1.2,1.0]$} \\
HW assignments & $43.1(5.8)$ & $33.7(10.4)$ & -1.0 & {$[-2.1,0.2]$} \\
Days worked on HW & $42.8(7.2)$ & $32.9(11.0)$ & -0.9 & {$[-2.1,0.2]$} \\
Session attendance & $6.5(1.0)$ & $5.5(1.1)$ & -0.9 & {$[-2.0,0.2]$} \\
\hline
\end{tabular}

Note. HW, homework; TOPF, Advanced Clinical Solutions: Test of Premorbid Functioning; RBANS A, Repeatable Battery for

Neuropsychological Status Update A; DKEFS, Delis-Kaplan Executive Function System; HADS, Hospital Anxiety and Depression Scale; GRQ, Group Readiness Questionnaire, Expectancy subscale; GCQ-S, Group Climate Questionnaire, short form. 


\section{Table 17}

Older Adult: Baseline Information for Homework Completion Groups in the Older Adult Sample

\begin{tabular}{|c|c|c|c|c|}
\hline Variable & $\begin{array}{l}\mathrm{HW}<50 \% \\
(n=8)\end{array}$ & $\begin{array}{l}\text { HW } 50 \%-74 \% \\
(n=8)\end{array}$ & $\begin{array}{l}\text { HW } 75 \%+ \\
(n=9)\end{array}$ & $\eta_{p}^{2}$ \\
\hline Age & $74.6(6.6)$ & $69.9(6.5)$ & $71.1(5.0)$ & 0.1 \\
\hline Gender ( $\%$ female $)$ & $38 \%$ & $63 \%$ & $67 \%$ & 0.1 \\
\hline Education & $17.0(3.0)$ & $17.5(2.6)$ & $15.8(3.4)$ & 0.1 \\
\hline TOPF & $112.5(9.3)$ & $116.6(7.2)$ & $117.4(5.1)$ & 0.1 \\
\hline RBANS A total index & $113.4(14.0)$ & $110.9(16.6)$ & $116.2(13.2)$ & 0.0 \\
\hline DKEFS inhibition & $10.1(3.0)$ & $9.3(3.7)$ & $10.4(2.6)$ & 0.0 \\
\hline DKEFS inhibition/switching & $10.8(2.6)$ & $9.0(4.2)$ & $11.3(1.7)$ & 0.1 \\
\hline DKEFS sorting & $13.1(3.8)$ & $12.8(2.6)$ & $14.1(3.0)$ & 0.0 \\
\hline HADS anxiety & $4.8(2.7)$ & $6.9(3.3)$ & $5.2(4.4)$ & 0.1 \\
\hline HADS depression & $2.3(1.6)$ & $4.5(2.7)$ & $3.2(2.4)$ & 0.1 \\
\hline GRQ-E & $8.6(2.8)$ & $7.9(1.1)$ & $6.2(2.9)$ & 0.2 \\
\hline GCQ-S Engagement subscale & $3.3(0.7)$ & $3.6(0.8)$ & $4.2(1.0)$ & 0.2 \\
\hline HW assignments & $13.3(8.6)$ & $33.3(3.4)$ & $43.6(4.8)$ & 0.8 \\
\hline Days worked on HW & $12.6(8.3)$ & $32.1(3.5)$ & $43.8(4.7)$ & 0.8 \\
\hline Session attendance & $3.6(1.7)$ & $5.3(0.7)$ & $6.6(0.9)$ & 0.6 \\
\hline
\end{tabular}

Note. HW, homework; TOPF, Advanced Clinical Solutions: Test of Premorbid Functioning; RBANS A, Repeatable Battery for Neuropsychological Status Update A; DKEFS, Delis-Kaplan Executive Function System; HADS, Hospital Anxiety and Depression Scale; GRQ, Group Readiness Questionnaire, Expectancy subscale; GCQ-S, Group Climate Questionnaire, short form 


\section{Aim 2: To provide Information Regarding Resources Required for EON-Mem}

Major costs and time required to complete the study are reported in Table 18.

Considering both cost for manuals and reimbursement as well as research assistant time, we estimated running one older adult participant in our study cost about $\$ 319$, itemized below:

- $\$ 141$ for reimbursement and materials

- $\$ 34$ for pre-test (\$10/hour*3.4 hours/participant)

- $\$ 34$ for post-test (\$10/hour*3.4 hours/participant)

- $\$ 3$ for research assistants being trained (\$10/hour*(8.0 total hours/26 participants))

- $\$ 1$ for research assistants training another (\$10/hour*(3.0 total hours/26 participants)

- $\$ 53$ for scoring and data entry (\$10/hour*(138.1 total hours/26 participants))

- $\$ 28$ for recruiting (\$10/hour*(72.9 total hours/26 participants))

- $\$ 8$ for fidelity checks (( $\$ 10 /$ hour*1.4 hour per session*5.2 sessions per group)/8.7 participants per group)

- $\$ 17$ for leading sessions ((\$20/hour*1.4 hour per session*5.2 sessions per group)/8.7 participants per group) 
EON-MEM FOR OLDER ADULTS

Table 18

Older Adult: Cost and Time Required for Implementation

\begin{tabular}{|c|c|c|}
\hline \multirow{2}{*}{$\begin{array}{l}\text { Variable } \\
\text { Total cost/participant (US \$) }\end{array}$} & Mean $(S D) /$ Percentage & Range \\
\hline & 319 & \\
\hline $\begin{array}{l}\text { Total reimbursement and materials (per } \\
\text { participant; US \$) }\end{array}$ & $141(27)$ & $64-166$ \\
\hline \multirow{2}{*}{$\begin{array}{l}\text { Reimbursement (per participant; US \$) } \\
\text { Protocols and manuals (per participant; } \\
\text { US \$) }\end{array}$} & $78(23)$ & $10-100$ \\
\hline & $64(5)$ & $54-66$ \\
\hline Total research time required (per participant; h) & 16.9 & \\
\hline Pre-testing (per participant; h) & $3.4(0.4)$ & $3.1-4.0$ \\
\hline Post-testing (per participant; h) & $3.4(0.5)$ & $3.0-3.9$ \\
\hline Being trained (per sample; $h$ ) & 8.0 & \\
\hline Training another (per sample; h) & 3.0 & \\
\hline Scoring and data entry (per sample; h) & 138.1 & \\
\hline Recruiting (per sample; $h$ ) & 72.9 & \\
\hline Session time (across sessions; $h$ ) & $1.4(0.2)$ & $0.7-1.7$ \\
\hline Session two (h) & $1.0(0.3)$ & $0.7-1.2$ \\
\hline Session three $(\mathrm{h})$ & $1.4(0.1)$ & $1.3-1.5$ \\
\hline Session four $(\mathrm{h})$ & $1.5(\mathrm{~N} / \mathrm{A})$ & $\mathrm{N} / \mathrm{A}$ \\
\hline Session five (h) & $1.5(0.1)$ & $1.4-1.6$ \\
\hline Session six (h) & $1.3(0.1)$ & $1.3-1.4$ \\
\hline Session seven $(\mathrm{h})$ & $1.5(\mathrm{~N} / \mathrm{A})$ & N/A \\
\hline Session eight $(\mathrm{h})$ & $1.7(\mathrm{~N} / \mathrm{A})$ & $\mathrm{N} / \mathrm{A}$ \\
\hline
\end{tabular}

Next, we estimated one participant required 16.9 hours of research assistant time, itemized below:

- $\quad 3.4$ hours for pre-test +3.4 hours for post-test

- $\quad 0.3$ hours for research assistant being trained (8.0 hours/26 participants)

- $\quad 0.1$ hours for research assistant training another (3.0 hours/26 participants) 
- $\quad 5.3$ hours for scoring and data entry (138.1 hours/26 participants)

- $\quad 2.8$ hours for recruiting (72.9 hours/26 participants)

- $\quad 0.8$ hours for fidelity checks ((1.4 hour per session*5.2 sessions per group)/ 8.7 participants per group)

- $\quad 0.8$ hours for leading sessions ((1.4 hour per session*5.2 sessions per group)/ 8.7 participants per group)

Importantly, visual inspection of Table 18 reveals only minimal training of research assistant for the study was required after initial training. Table 18 also reveals a significant amount of time was spent in recruiting. Finally, unlike younger adults, sessions with older adults most often required the full 90 minutes (see Figure 15 for duration of specific sessions). 


\section{Figure 15}

Older Adult: Average Session Length

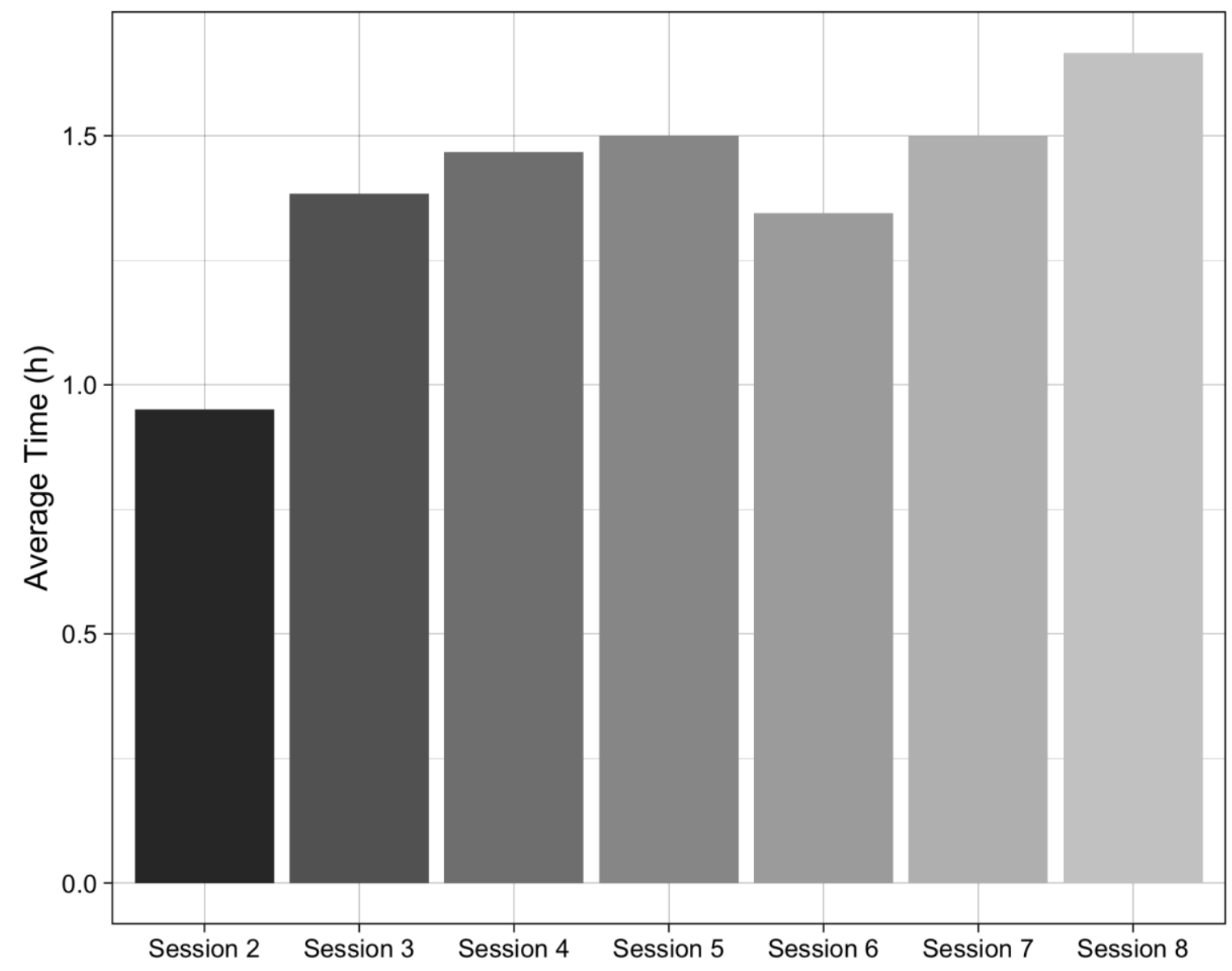

Regarding recruitment, we contacted 175 older adults interested in the study. One hundred fortynine of the participants ( $85 \%)$ were excluded from participation. The most common reason for exclusion was no response to contact ( $n=60)$, likely because we used prior databases for contact. The second most common reason for exclusion was timing conflicts with the treatment dates $(n=$ 32 ), followed closely by too young to participate in the study ( $<65$ years; $n=25$ ). Other reasons included no longer interested, poor health, IQ code score, non-native English speaker, and COVID-related cancellations. Overall, $15 \%$ of the original contacted pool of older adults 
interested in the study were enrolled, with no response and timing conflicts being the most common reasons for exclusion.

\section{Aim 3: To Assess Change in Memory Performance}

Only four of the 20 older adult participants who received both pre- and post-intervention tests $(20 \%$ of the sample) were considered to have improved by the end of the intervention according to the RCI criteria for the RBANS memory index (see Figure 16). Two of the older adults had reliably worse scores on the RBANS memory index after the intervention, and the majority demonstrated no reliable change on the RBANS memory index after the intervention (14; Figure 16). On the RBMT, two older adults had reliably better scores, five older adults had reliably worse scores, and 14 older adults demonstrated no change after intervention (Figure 16). Regarding Everyday Memory Simulations subtests, we observed the most variability on the Everyday Memory Simulations biographical subtest for face and names (five performed reliably better and nine performed reliably worse after intervention). We observed the most improvement on the Everyday Memory Simulations message subtest (eight older adults had reliably better scores and only one older adult had a reliably worse score after treatment). Finally, we observed the most decline in performance on the Everyday Memory Simulations object location subtest (one participant performed reliably better after intervention and three participants performed reliably worse). Overall, however, $73 \%$ of participants demonstrated no reliable change on memory indices after intervention (Figure 16). 


\section{Figure 16}

Older Adult: RCIfor Memory Indices

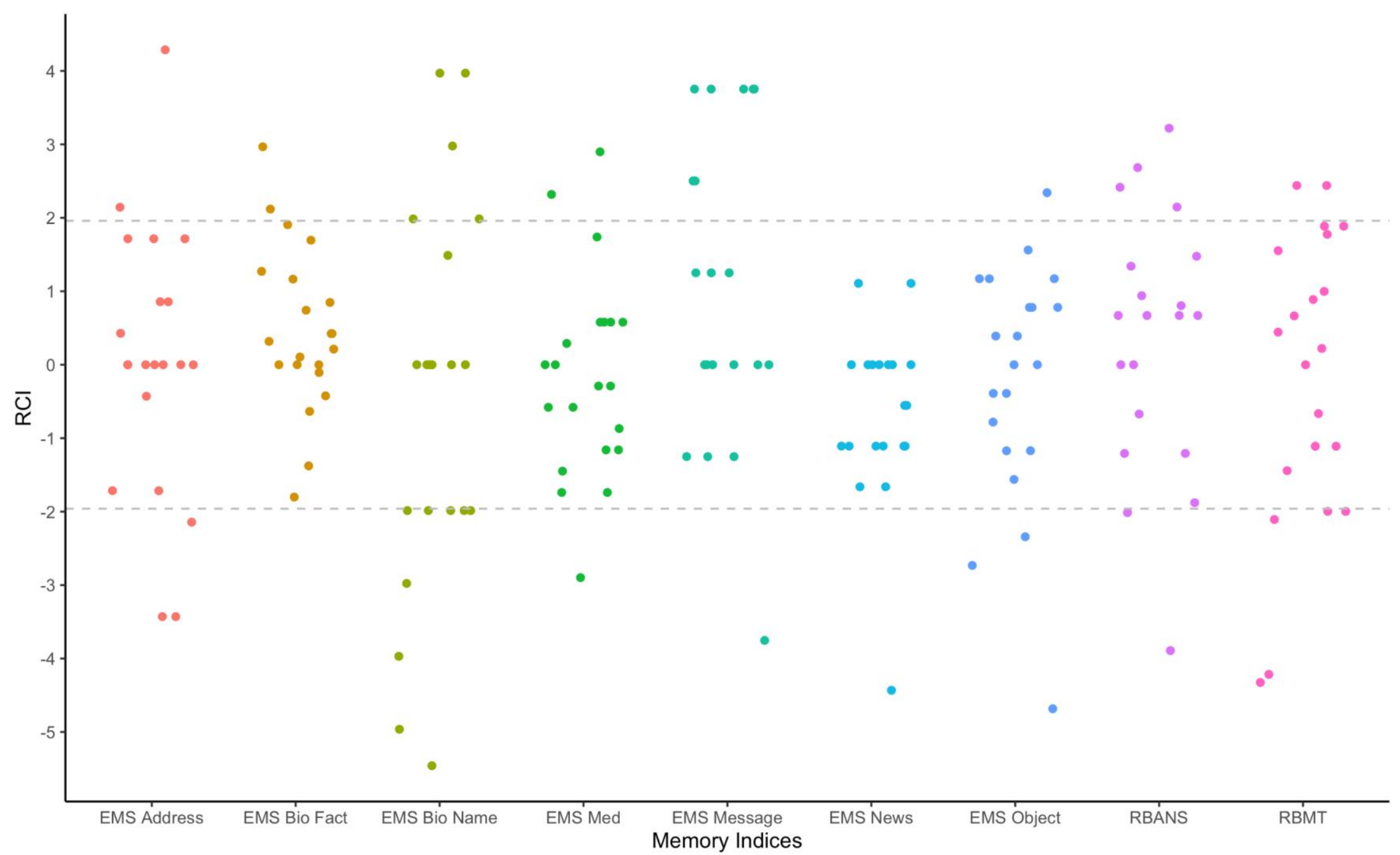


Ninety percent of older adults showed no reliable change between pre- and postintervention on RBANS subtests, although $7 \%$ of older adults showed reliable improvement and $3 \%$ of older adults showed reliable decline for subtests in the RBANS (see Figure 17). We observed the most variability on the RBANS coding and figure copy subtests (for each subtest, three older adults had reliably better scores and one older adult had a reliably worse score after intervention). We observed the most improvement on RBANS story immediate recall (three older adults had reliably better scores and no participants had reliably worse scores after treatment). Finally, we observed the most decline in performance on the RBANS semantic fluency (no older adults had reliably better scores and three older adults had reliably worse scores after treatment). Overall, however, most older adults (90\%) demonstrated no reliable change on RBANS subtests after intervention (Figure 17). 


\section{Figure 17}

Older Adult: RCI for RBANS Subtests

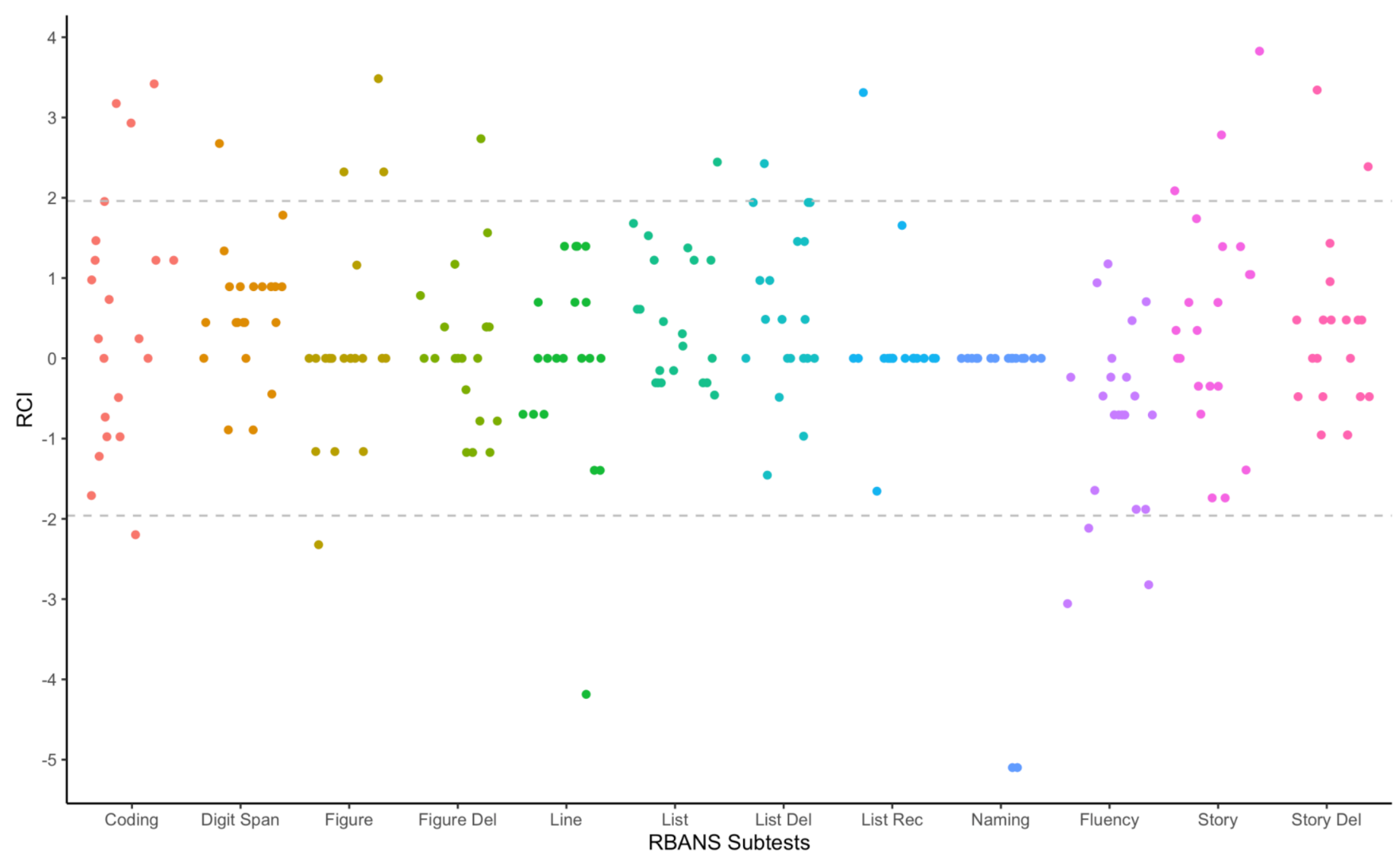




\section{Older Adult: Discussion}

Implementation of EON-Mem in the older adult sample provided important information about the feasibility of a clinical trial. Although the study provided important information, policy changes related to COVID-19 likely impacted the attendance and performance of older adult participants in our study. When excluding the sessions affected by COVID-19 policy, participants averaged $85 \%$ treatment attendance rate. However, according to the original study definition, and not accounting for COVID-19 policy changes, the completion rate was quite low (38\% completers). Regarding homework, older adult participants on average completed $62 \%$ of all homework assigned. Homework completion rates averaged $72 \%$ when considering sessions prior to COVID-19 related changes in policy. Twenty percent of the older adult sample improved as measured by the RBANS memory index, $10 \%$ performed reliably worse, and $70 \%$ of older adults had no reliable change between pre- and post-intervention assessment. A similar pattern of change was found on the other memory and cognitive tests: overall, $83 \%$ of older adults did not reliably change on memory and cognitive tests over time. The few who did improve on the RBANS memory index in our study had higher predicted intelligence, lower cognitive skills, lower inhibition skills, higher depression, and higher homework and session completion compared to those who did not improve. Although costs and time decreased with revisions made from the young adult sample, the study still required $\$ 319 /$ participant and 16.9 hours of research assistant time/participant. For recruitment, $15 \%$ of the original contacted pool of older adults interested in the study were enrolled in the study, with no response and timing conflicts being the most common reasons for exclusion. Overall, implementation of EON-Mem in older adults demonstrated an acceptable treatment adherence rate (when taking COVID-19 into account), but 
a minority of older adults improved (20\%) in memory and implementation of the study required higher than expected costs.

The attendance patterns observed for older adults in our study shed light on how attendance could have been different had the study not occurred during the COVID-19 pandemic. It is likely completion rate would have been within a priori expectations $(75 \%)$ had the last one to two treatment sessions in two of the groups not been affected by COVID-19. For example, prior to COVID-19 related changes in policy, $58 \%$ of the sample had perfect attendance and $19 \%$ of the sample missed only one session, leaving $77 \%$ eligible for a "completer" status at the end of treatment. Further, older adults demonstrated high postintervention test attendance (81\%). Older adult attendance patterns (e.g., no older adult participant attended only one or two sessions) also suggest commitment to treatment. Finally, older adults reported no problems with scheduling sessions. Overall, we expect attendance in the older sample would have approached expected rates ( $75 \%$ completers) had the final sessions not been affected by COVID-19.

Another takeaway from the older adult sample was the significant heterogeneity between individuals in the older adult groups/sample. First, older adult opinions regarding the usefulness of homework varied greatly between participants, ranging from strongly endorsing the homework utility to somewhat disagreeing that the homework was useful. Second, older adults had quite variable homework completion (mean: 30.6, range: 0-49, standard deviation: 14.1). Notably, some of this variability could be associated with the complaints about difficulty working with the take-home materials (e.g., adapted homework book difficult to navigate, unable to refer to slides at home). Third, older adults held various opinions of the content/sessions enjoyed and those they disliked, although a large portion disliked peg words. Heterogeneity is 
something we would expect in group treatment, and is an inherent characteristic to be aware of in teaching a group: the same homework assignment can be too easy for one participant and too hard for another participant. The same applies to usefulness of content and homework, depending on the participant's approach to learning information. Thus, the variability in opinion is not surprising in our sample. As such, future studies might consider individual treatment as opposed to group treatment. Despite differing in what they wanted to learn, most participants enjoyed how the material was presented, including both therapist presentation and the group dynamic.

Memory performance as measured by the RBANS did not reliably change after intervention for most (70\%) older adults, however memory did reliably improve for $20 \%$ of the older adults. Participants who improved in our sample had higher predicted intelligence, but lower general cognitive performance, including lower executive functioning. Perhaps EON-Mem was helpful to participants who were underperforming in life, that is, had high capabilities but specific deficits in performance. Specifically, the compensatory treatment may have been especially helpful to those who had difficulty imposing a structure independently, considering those that improved had somewhat lower executive functioning skills than those who did not improve. Participants who improved slightly were also more anxious and depressed at baseline compared to those who did not improve; maybe the socialization and schedule inherent in a group treatment setting alleviated mood and anxiety symptoms, indirectly supporting improved cognitive performance. Finally, the higher attendance and homework rates for improvers is promising for the efficacy of the treatment, although certainly not indicative of effectiveness. 


\section{Overall Discussion}

The goal of our study was to determine if it was feasible to perform an RCT of EONMem in group-format with older adults. Throughout the discussion section, we will first comment on similar patterns found between the young and older adult sample. We will then discuss differences between young and older adult samples. Next, we will provide support for and against running an RCT of EON-Mem in groups of older adults. Finally, we will comment on strengths and limitations of the current study.

We found a few themes common to both samples (older and younger adults) in the implementation of EON-Mem. First, we found improvement rates were the same across groups (20\%). Participants in both age groups who improved in our study had lower cognitive functioning than those who did not improve. This is perhaps suggestive of the limitations of EON-Mem in high-functioning groups, especially for those who are already performing as expected based on intellectual functioning. Costs for administering the study were also similar across samples and higher than expected. In recruitment, although the retention rate from initial contact to enrollment was different between young and older adults (35\% vs. 15\%, respectively), the most common reasons for exclusion in both samples were lack of response and timing conflicts. Next, relationships between group members and with the therapist were positive the majority of the time, and even higher in the young adult improvers compared to non-improvers, as measured by the GCQ. Both samples also appreciated the presentation of the content, although both samples desired more clarity on requirements prior to attending. Diverse opinions about the content (which strategies participants liked) was present in each sample, yet both samples agreed on disliking peg words. 
We also observed differences in implementation of EON-Mem between older adult and younger adult samples. When taking COVID-related impacts into account, older adults had higher session and homework completion rates compared to young adult groups. This may be explained by the apathy and amotivation in the young adult sample that was not reported in the older adult sample. Further, based on older adult attendance patterns (only $4 \%$ of older adults dropped out after two sessions, whereas $23 \%$ of the young adult group dropped out after two sessions), it seems older adults were more willing to give EON-Mem a try. Average session time was somewhat longer with the older adult sample compared to the younger adult sample, as expected (1.4 hours compared to 1.0 hours). Older adults and younger adults made different complaints: older adults were distracted/frustrated by talkative and tangential group members and found using the physical material to be confusing at times; younger adults complained of variable reminder text times and a majority disliked the homework.

Considering information gained across both samples and all groups, we considered the question, is an RCT of group-format EON-Mem feasible and worthwhile in a sample of older adults? Some factors indicate promise for an RCT of group-format EON-Mem in older adults. In our sample, older adults reported enjoying the group dynamic and presentation of the material. Further, $77 \%$ of the older adult sample was eligible for completer status had the final sessions not been cancelled due to COVID-19. Next, because many older adults were not motivated by money, compensation could be reduced to make the study more affordable. Further, future studies may reduce the number of memory tests included in the battery, cutting down on protocol cost and administration and data entry time.

However, some information would suggest an RCT of EON-Mem in group-format for older adults is not feasible or practical. The improvement rates were $20 \%$ in both of our young 
adult and older adult samples based on the RBANS Memory Index. In order to bench-mark improvement rates in our samples against previous, similar treatments, we referred to Estrada, Ferrer, and Pardo (2018). Estrada et al. (2018) argue a net percentage of change can be estimated from effect size; from their simulated scenarios with single-group, pre-post designs, a small effect size corresponded to $8 \%$ of changes, a medium effect size corresponded to $17 \%$, and a large effect size corresponded to $26 \%$ of changes. In our study, the net percentage of reliable change was $10 \%$ (20\% reliably improved $-10 \%$ reliably declined), corresponding to a small effect size (Estrada et al., 2018). Similarly, current meta-analyses suggest compensatory memory programs in older adults are associated with a small-to-medium effect size (Gross et al., 2012; Verhaeghen et al., 1992). Of note, although the net change in our study may be comparable to other effect sizes, such a small net change in the sample may or may not be worthwhile for future researchers. Finally, the effect of the intervention from our sample should not be generalized.

Because the intervention focused on compensatory skills to be used in everyday situations, we expected participants would perform better on tests with higher ecological validity (i.e., Everyday Memory Simulations). However, in our young adult sample, improvement rates as measured by the Everyday Memory Simulations subtests (average: 9\%, range: 0\%-20\%) were not higher than improvement rates as measured by the RBANS Memory Index. Improvement rates across memory measures were also similar for our older adult sample $(20 \%$ improvement using the RBANS Memory Index; average 14\% improvement (range: 0\%-38\%) using the Everyday Memory Simulations subtests).

Other than improvement rates, there are a few additional reasons researchers may find a future trial impractical. First, in our sample, EON-Mem was especially beneficial for those functioning poorly in every-day life (improvers had lower cognitive functioning and executive 
skills than those that did not improve); perhaps most healthy older adults are functioning at a level too high to benefit from the strategies taught in EON-Mem. Next, the study cost (\$319 and 16.9 research hours per participant) may or may not be feasible depending on a researcher's funding. Further, $85 \%$ of the interested older adults contacted were not enrolled in the study and recruitment alone required 72.9 research hours. Although some costs may be cut (e.g., exclude protocols from battery, reduce compensation), many costs are non-negotiable (e.g., EON-Mem manuals are copy-righted, data entry and recruitment required for a rigorously-completed study). Finally, cutting down on compensation would likely lead to increased time spent on recruitment. In considering the arguments above, yes, an RCT of EON-Mem in group-format with older adults is feasible: we expect a majority (75\%) of older adults would attend treatment and testing sessions, older adults would enjoy the group dynamic, and the intervention would be implemented without any major obstacles (e.g., therapist barriers to administration of treatment, lack of materials, timing conflicts, recruitment barriers) excepting, of course, a global pandemic. However, the answer to whether or not an RCT is worthwhile is in the eye of the beholder/researcher. The individual RCIs calculated in the current study demonstrated that memory scores of a few individuals improved over time, but this information cannot be interpreted as intervention effectiveness; thus, the potential researcher remains about as certain or uncertain about EON-Mem effectiveness as she/he did in the introduction of this dissertation. However, the feasibility study did shed light on the cost of a potential RCT of EON-Mem in older adults. Based on an estimated required sample size of 144 (from small-to-medium effect sizes found in similar treatments), we anticipate the total required cost of $\$ 45,936$ and 2,434 research hours for a full RCT (not including PI, co-I, or consultant salaries). Such costs may or may not be affordable, depending on the researcher. Reliable and significant change even for a 
minority of participants (20\%) is likely worthwhile clinically considering the potential impact of improved memory skills on quality of life. However, the number of empirically-based memory trainings already in existence for older adults may obviate the need for a full clinical trial of EON-Mem. Thus, an RCT of group-format EON-Mem in older adults is feasible but may or may not be worthwhile depending on the researcher's resources and goals.

The feasibility study provides valuable information to inform potential future studies and had several strengths. The study used multiple methods for measuring memory (RBANS, RBMT, Everyday Memory Simulations) allowing us to triangulate change in improvement and speculate potential differences between types of memory measures. The study also utilized several means of gathering feedback (i.e., individual interviews, group interviews, weekly questionnaires) enabling us to gather several suggestions and themes for change. We used several procedures to improve data integrity (e.g., score checks, trainings, double entry) which strengthened the statistical validity of the study. Finally, the study had a strength in ecological validity, not just in measures used, but in the sample recruited (community sample of older adults) and intervention (flexible homework application, group membership).

The feasibility study also had limitations. First, because many of the participants who stopped attending treatment do not attend the post-intervention test and exit interview, our results are likely skewed toward positive comments and changes. In order to address the limitation, we followed up with participants and offered compensation for a post-test (including an exit interview), likely contributing to the low measurement attrition in the study. The lack of established psychometrics for Everyday Memory Simulations (especially test-retest information) led to missing the reliability data required to accurately compute RCIs. Although we substituted internal reliability in the current study and performed a sensitivity analysis using similar 
measures, the lack of psychometric information for Everyday Memory Simulations limits our ability to interpret the results. Next, the primary memory measure used to calculate RCI (RBANS) is not ecologically-oriented and likely precludes participants from employing many of the skills learned during treatment, perhaps suggesting lower treatment efficacy than exists. Although, this is unlikely as we also calculated RCI of Everyday Memory Simulations and found a similar pattern of results. The current study did not include a quality of life measure, potentially missing important changes in participants' functioning not measured by memory scores. Finally, a major limitation to the study was the impact of COVID-19, leading to cancelled sessions and early testing, associated with lower attendance rates and variable testing effects on each group. In order to compensate, we included pre-COVID estimates and offered testing soon after, reducing the potential measurement attrition.

Implementation of group-format EON-Mem in groups other than healthy older adults may be warranted. Considering the trends found in our sample, EON-Mem may hold promise for participants with low executive functioning and/or cognition and/or high depression. Future studies might use a screener to identify participants with low executive functioning skills and/or low cognition and/or high depression. Future research may even target participants with particular diagnoses often associated with the deficits described above, such as attention-deficit disorder or traumatic brain injury. In research with clinical and healthy populations, one might also consider implementation of EON-Mem in or with other formats, such as apps, tele-health, and video games.

I recommend several changes to any future implementation of EON-Mem in order to improve the experience and usability of the treatment. First, I recommend adapting material in order to be more user-friendly. Examples include marking the homework pages with a paperclip 
to be done that week and suggesting at the beginning of treatment that group members take notes, as slides and therapist manual are not available. Future studies might also consider removing content on peg words as many group members found them too confusing. Next, I would train therapists to establish expectations early on in the group treatment, such as raising one's hand to speak and answering long questions after the class period in order to reduce distractions. An emailed copy of a syllabus stating class content and participant expectations would likely reduce participant confusion and improve attendance rates.

I also recommend methodological changes. First, a control group would be imperative not only for ruling out confounds, but to calculate test-retest reliabilities for Everyday Memory Simulations; use of Everyday Memory Simulations as a primary measure would likely be a better indicator of improvement as many EON-Mem strategies cannot be directly applied to more traditional memory measures. Next, considering the inherent heterogeneity and distractions in group settings as found in our study, future researchers may prefer to teach EON-Mem in an individual format. Additionally, future studies might consider using a subjective report of memory as we would expect changes in day-to-day life in an ecologically-oriented treatment. Finally, future research ought to include quality of life measures. Many traditional, and even ecological, measures miss the broader picture of participants' and patients' lives and functioning. Considering the goal of any clinical trial is to ultimately improve quality of life, we suggest including primary quality of life measures in a clinical trial. The quality of life measure selected depends on the target population. Examples of quality of life measures include EuroQol-5 Dimension (EQ-5D; a generic measure of health status) and the World Health Organization Quality of Life (WHOQOL; assesses physical health, psychological health, relationships, and environment; Devlin \& Brooks, 2017; Skevington et al., 2004). 
In summary, results from the current study suggest an RCT of group-format EON-Mem in older adults is feasible, but may or may be not cost-effective depending on the researcher's resources and goals. The number of already-established memory compensatory programs may weaken the rationale for conducting a large and expensive trial. One might consider conducting an RCT of EON-Mem in an area with fewer supported treatments (e.g., populations, alternative delivery methods) such as traumatic brain injury, mild cognitive impairment, epilepsy, or stroke. The information on cost, measurement tools, potential predictors of improvement, and areas of participant feedback will help prepare a researcher for implementation of EON-Mem or other similar memory-compensatory treatment programs in any population. 


\section{References}

Arnold, D. M., Burns, K. E., Adhikari, N. K., Kho, M. E., Meade, M. O., Cook, D. J., \& McMaster Critical Care Interest, G. (2009). The design and interpretation of pilot trials in clinical research in critical care. Crit Care Med, 37(1 Suppl), S69-74. doi:10.1097/CCM.0b013e3181920e33

Barman, A., Chatterjee, A., \& Bhide, R. (2016). Cognitive impairment and rehabilitation strategies after traumatic brain injury. Indian Journal of Psychological Medicine, 38(3), 172-181. doi:10.4103/0253-7176.183086

Bierman, E. J., Comijs, H. C., Jonker, C., \& Beekman, A. T. (2005). Effects of anxiety versus depression on cognition in later life. Am J Geriatr Psychiatry, 13(8), 686-693. doi:10.1176/appi.ajgp.13.8.686

Bjelland, I., Dahl, A. A., Haug, T. T., \& Neckelmann, D. (2002). The validity of the Hospital Anxiety and Depression Scale. An updated literature review. J Psychosom Res, 52(2), 6977.

Bottiroli, S., Cavallini, E., Dunlosky, J., Vecchi, T., \& Hertzog, C. (2017). Self-guided strategyadaption training for older adults: Transfer effects to everyday tasks. Arch Gerontol Geriatr, 72, 91-98. doi:10.1016/j.archger.2017.05.015

Burlingame, G. M., Davies, D., Cox, D., Baker, E., Pearson, M., Beecher, M., \& Gleave, R. (2012). The group readiness questionnaire manual. Salt Lake City, UT: OQ Measures.

Burlingame, G. M., McClendon, D. T., \& Yang, C. (2018). Cohesion in group therapy: A metaanalysis. Psychotherapy, 55(4), 384-398. doi:10.1037/pst0000173

Burlingame, G. M., Strauss, B., \& Joyce, A. S. (2013). Change mechanisms and effectiveness of 
small group treatments. In M. J. Lambert (Ed.), Bergin and Garfield handbook of psychotherapy and behavior change (pp. 640-690). Hoboken, NJ: John Wiley \& Sons, Incorporated.

Burlingame, G. M., Whitcomb, K. E., Woodland, S. C., Olsen, J. A., Beecher, M., \& Gleave, R. (2018). The effects of relationship and progress feedback in group psychotherapy using the Group Questionnaire and Outcome Questionnaire-45: A randomized clinical trial. Psychotherapy (Chic), 55(2), 116-131. doi:10.1037/pst0000133

Burt, D. B., Zembar, M. J., \& Niederehe, G. (1995). Depression and memory impairment: A meta-analysis of the association, its pattern, and specificity. Psychological Bulletin, 117(2), 285-305. doi:10.1037/0033-2909.117.2.285

Chan, A. S., Cheung, W. K., Yeung, M. K., \& Lee, T. L. (2018). Sustained effects of memory and lifestyle interventions on memory functioning of older adults: An 18-month followup study. Front Aging Neurosci, 10, 240. doi:10.3389/fnagi.2018.00240

Christensen, L., \& Mendoza, J. L. (1986). A method of assessing change in a single subject: An alteration of the RC index. Behavior Therapy, 15, 305-308.

Chu, Y., Lai, M. H. C., Xu, Y., \& Zhou, Y. (2012). Test review: Advanced clinical solutions for WAIS-IV and WMS-IV. Journal of Psychoeducational Assessment, 30(5), 520-524. doi: $10.1177 / 0734282912442868$

das Nair, R., Bradshaw, L. E., Day, F. E., Drummond, A., Harris, S. R., Fitzsimmons, D., .. . Lincoln, N. B. (2019). Clinical and cost effectiveness of memory rehabilitation following traumatic brain injury: A pragmatic cluster randomized controlled trial. Clin Rehabil, 33(7), 1171-1184. doi:10.1177/0269215519840069

Delis, D. C., Kaplan, E., \& Kramer, J. H. (2001). Delis-Kaplan executive function system: Technical manual. San Antonio, TX: Harcourt Assessment Company. 
Devlin, N. J., \& Brooks, R. (2017). EQ-5D and the EuroQol group: Past, present and future. Appl Health Econ Health Policy, 15(2), 127-137. doi:10.1007/s40258-017-0310-5

Eichenbaum, H. (2000). A cortical-hippocampal system for declarative memory. Nat Rev Neurosci, 1(1), 41-50. doi:10.1038/35036213

Ericsson, K. A. (1985). Memory skill. Canadian Journal of Psychology/Revue Canadienne de Psychologie, 39(2), 188-231. doi:10.1037/h0080059

Estrada, E., Ferrer, E., \& Pardo, A. (2018). Statistics for evaluating pre-post change: Relation between change in the distribution center and change in the individual scores. Front Psychol, 9, 2696. doi:10.3389/fpsyg.2018.02696

Fairchild, J. K., \& Scogin, F. R. (2010). Training to enhance adult memory (TEAM): An investigation of the effectiveness of a memory training program with older adults. Aging Ment Health, 14(3), 364-373. doi:10.1080/13607860903311733

Frankenmolen, N. L., Overdorp, E. J., Fasotti, L., Claassen, J., Kessels, R. P. C., \& Oosterman, J. M. (2018). Memory strategy training in older adults with subjective memory complaints: A randomized controlled trial. J Int Neuropsychol Soc, 24(10), 1110-1120. doi:10.1017/S1355617718000619

Fritsch, T., McClendon, M. J., Wallendal, M. S., Hyde, T. F., \& Larsen, J. D. (2014). Prevalence and cognitive bases of subjective memory complaints in older adults: Evidence from a community sample. J Neurodegener Dis, 2014, 176843. doi:10.1155/2014/176843

Goedendorp, M. M., Kuiper, D., Reijneveld, S. A., Sanderman, R., \& Steverink, N. (2017). Sustaining program effectiveness after implementation: The case of the self-management of well-being group intervention for older adults. Patient Educ Couns, 100(6), 11771184. doi:10.1016/j.pec.2017.01.006 
Gontkovsky, S. T., Beatty, W. W., \& Mold, J. W. (2004). Repeatable battery for the assessment of neuropsychological status in a normal, geriatric sample. Clinical Gerontologist, 27(3), 79-86. doi:10.1300/J018v27n03_07

Gross, A. L., Parisi, J. M., Spira, A. P., Kueider, A. M., Ko, J. Y., Saczynski, J. S., . . Rebok, G. W. (2012). Memory training interventions for older adults: A meta-analysis. Aging Ment Health, 16(6), 722-734. doi:10.1080/13607863.2012.667783

Harter, M., Woll, S., Wunsch, A., Bengel, J., \& Reuter, K. (2006). Screening for mental disorders in cancer, cardiovascular and musculoskeletal diseases. Comparison of HADS and GHQ-12. Soc Psychiatry Psychiatr Epidemiol, 41(1), 56-62. doi:10.1007/s00127005-0992-0

Hawley, K. S., \& Cherry, K. E. (2008). Memory interventions and quality of life for older adults with dementia. Activities, Adaptation \& Aging, 32(2), 89-102. doi:10.1080/01924780802142958

Hertzog, C., Kramer, A. F., Wilson, R. S., \& Lindenberger, U. (2008). Enrichment effects on adult cognitive development: Can the functional capacity of older adults be preserved and enhanced? Psychological Science in the Public Interest, 9(1), 1-65. doi:10.1111/j.15396053.2009.01034.x

Hudes, R., Rich, J. B., Troyer, A. K., Yusupov, I., \& Vandermorris, S. (2019). The impact of memory-strategy training interventions on participant-reported outcomes in healthy older adults: A systematic review and meta-analysis. Psychol Aging, 34(4), 587-597. doi:10.1037/pag0000340

Jacobson, N. S., \& Truax, P. (1991). Clinical significance: A statistical approach to defining meaningful change in psychotherapy research. J Consult Clin Psychol, 59(1), 12-19. 
Jonker, C., Geerlings, M. I., \& Schmand, B. (2000). Are memory complaints predictive for dementia? A review of clinical and population-based studies. Int J Geriatr Psychiatry, 15(11), 983-991.

Jorm, A. F. (1994). A short form of the informant questionnaire on cognitive decline in the elderly (IQCODE): Development and cross-validation. Psychol Med, 24(1), 145-153.

Julious, S. A. (2005). Sample size of 12 per group rule of thumb for a pilot study. Pharmaceutical Statistics, 4(4), 287-291. doi:10.1002/pst.185

Karr, J. E., Hofer, S. M., Iverson, G. L., \& Garcia-Barrera, M. A. (2018). Examining the latent structure of the Delis-Kaplan Executive Function System. Arch Clin Neuropsychol, 34(3), 381-394. doi:10.1093/arclin/acy043

Kivlighan, D. M., Jr., \& Goldfine, D. C. (1991). Endorsement of therapeutic factors as a function of stage of group development and participant interpersonal attitudes. Journal of Counseling Psychology, 38(2), 150-158. doi:10.1037/0022-0167.38.2.150

Klingberg, T. (2010). Training and plasticity of working memory. Trends Cogn Sci, 14(7), 317324. doi:10.1016/j.tics.2010.05.002

Knight, R. G., McMahon, J., Green, T. J., \& Skeaff, C. M. (2006). Regression equations for predicting scores of persons over 65 on the Rey Auditory Verbal Learning Test, the MiniMental State Examination, the Trail Making Test and semantic fluency measures. $\mathrm{Br} J$ Clin Psychol, 45(Pt 3), 393-402.

Leon, A. C., Davis, L. L., \& Kraemer, H. C. (2011). The role and interpretation of pilot studies in clinical research. J Psychiatr Res, 45(5), 626-629. doi:10.1016/j.jpsychires.2010.10.008 
MacKenzie, K. R. (1983). Clinical application of a Group Climate Measure. In R. R. D. K. R. MacKenzie (Ed.), Advances in group psychotherapy: Integrating research and practice. (pp. 159-170). New York, NY: International Universities Press.

Mahncke, H. W., Connor, B. B., Appelman, J., Ahsanuddin, O. N., Hardy, J. L., Wood, R. A., . . . Merzenich, M. M. (2006). Memory enhancement in healthy older adults using a brain plasticity-based training program: A randomized, controlled study. Proc Natl Acad Sci U $S A, 103(33), 12523-12528$. doi:10.1073/pnas.0605194103

McDougall, G. J., Jr., Becker, H., Pituch, K., Acee, T. W., Vaughan, P. W., \& Delville, C. L. (2010). The SeniorWISE study: Improving everyday memory in older adults. Arch Psychiatr Nurs, 24(5), 291-306. doi:10.1016/j.apnu.2009.11.001

Montejo, P., Montenegro, M., Fernandez, M. A., \& Maestu, F. (2011). Subjective memory complaints in the elderly: Prevalence and influence of temporal orientation, depression and quality of life in a population-based study in the city of Madrid. Aging \& Mental Health, 15(1), 85-96. doi:10.1080/13607863.2010.501062

Montejo, P., Montenegro, M., Fernandez, M. A., \& Maestu, F. (2012). Memory complaints in the elderly: Quality of life and daily living activities. A population based study. Arch Gerontol Geriatr, 54(2), 298-304. doi:10.1016/j.archger.2011.05.021

Oh, S. J., Seo, S., Lee, J. H., Song, M. J., \& Shin, M. S. (2018). Effects of smartphone-based memory training for older adults with subjective memory complaints: A randomized controlled trial. Aging Ment Health, 22(4), 526-534.

doi:10.1080/13607863.2016.1274373 
Old, S. R., \& Naveh-Benjamin, M. (2008). Differential effects of age on item and associative measures of memory: A meta-analysis. Psychol Aging, 23(1), 104-118. doi:10.1037/0882-7974.23.1.104

Pandharipande, P. P., Girard, T. D., Jackson, J. C., Morandi, A., Thompson, J. L., Pun, B. T., . . Investigators, B.-I. S. (2013). Long-term cognitive impairment after critical illness. $N$ Engl J Med, 369(14), 1306-1316. doi:10.1056/NEJMoa1301372

Perez, M., \& Godoy, J. (1998). Comparison between a "traditional" memory test and a "behavioral" memory battery in Spanish patients. J Clin Exp Neuropsychol, 20(4), 496502. doi:10.1076/jcen.20.4.496.1478

Prull, M. W., Gabrieli, J. D. E., \& Bunge, S. A. (2000). Age-related changes in memory: A cognitive neuroscience perspective. In F. I. M. Craik \& T. A. Salthouse (Eds.), The handbook of aging and cognition, 2nd ed. (pp. 91-154). Mahwah, NJ.: Lawrence Erlbaum Associates Publishers.

Ptak, R., der Linden, M. V., \& Schnider, A. (2010). Cognitive rehabilitation of episodic memory disorders: from theory to practice. Front Hum Neurosci, 4. doi:10.3389/fnhum.2010.00057

Randolph, C., Tierney, M. C., Mohr, E., \& Chase, T. N. (1998). The Repeatable Battery for the Assessment of Neuropsychological Status (RBANS): Preliminary clinical validity. J Clin Exp Neuropsychol, 20(3), 310-319. doi:10.1076/jcen.20.3.310.823

Rattenborg, N. C., \& Martinez-Gonzalez, D. (2011). A bird-brain view of episodic memory. Behavioural Brain Research, 222(1), 236-245. doi:https://doi.org/10.1016/j.bbr.2011.03.030 
Rebok, G. W., Ball, K., Guey, L. T., Jones, R. N., Kim, H. Y., King, J. W., . . Willis, S. L. (2014). Ten-year effects of the ACTIVE cognitive training trial on cognition and everyday functioning in older adults. Journal of the American Geriatrics Society, 62(1), 16-24. doi:10.1111/jgs.12607

Rebok, G. W., Carlson, M. C., \& Langbaum, J. B. (2007). Training and maintaining memory abilities in healthy older adults: Traditional and novel approaches. J Gerontol B Psychol Sci Soc Sci, 62 Spec No 1, 53-61.

Rebok, G. W., Langbaum, J. B. S., Jones, R. N., Gross, A. L., Parisi, J. M., Spira, A. P., . . Brandt, J. (2013). Memory training in the ACTIVE study: How much is needed and who benefits? Journal of Aging and Health, 25(0). doi:10.1177/0898264312461937

Rogers, W. A., Hertzog, C., \& Fisk, A. D. (2000). An individual differences analysis of ability and strategy influences: Age-related differences in associative learning. Journal of Experimental Psychology: Learning, Memory, and Cognition, 26(2), 359-394. doi:10.1037/0278-7393.26.2.359

Rosi, A., Del Signore, F., Canelli, E., Allegri, N., Bottiroli, S., Vecchi, T., \& Cavallini, E. (2018). The effect of strategic memory training in older adults: Who benefits most? Int Psychogeriatr, 30(8), 1235-1242. doi:10.1017/S1041610217002691

Schnitzspahn, K. M., Stahl, C., Zeintl, M., Kaller, C. P., \& Kliegel, M. (2013). The role of shifting, updating, and inhibition in prospective memory performance in young and older adults. Dev Psychol, 49(8), 1544-1553. doi:10.1037/a0030579

Sim, J., \& Lewis, M. (2012). The size of a pilot study for a clinical trial should be calculated in relation to considerations of precision and efficiency. J Clin Epidemiol, 65(3), 301-308. doi:10.1016/j.jclinepi.2011.07.011 
Simons, D. J., Boot, W. R., Charness, N., Gathercole, S. E., Chabris, C. F., Hambrick, D. Z., \& Stine-Morrow, E. A. (2016). Do "brain-training" programs work? Psychol Sci Public Interest, 17(3), 103-186. doi:10.1177/1529100616661983

Skevington, S. M., Lotfy, M., O'Connell, K. A., \& Group, W. (2004). The World Health Organization's WHOQOL-BREF quality of life assessment: Psychometric properties and results of the international field trial. A report from the WHOQOL group. Qual Life Res, 13(2), 299-310. doi:10.1023/B:QURE.0000018486.91360.00

Smith, G. E., Housen, P., Yaffe, K., Ruff, R., Kennison, R. F., Mahncke, H. W., \& Zelinski, E. M. (2009). A cognitive training program based on principles of brain plasticity: Results from the Improvement in Memory with Plasticity-based Adaptive Cognitive Training (IMPACT) study. J Am Geriatr Soc, 57(4), 594-603. doi:10.1111/j.15325415.2008 .02167

Snaith, R. P. (2003). The Hospital Anxiety And Depression Scale. Health Qual Life Outcomes, 1, 29. doi:10.1186/1477-7525-1-29

Sporner, M. L. (2013). Evaluation of telerehabilitation for the delivery of remote cognitive rehabilitation. Unpublished doctoral dissertation. University of Pittsburgh.

Stringer, A. Y. (2011). Ecologically-oriented neurorehabilitation of memory: Robustness of outcome across diagnosis and severity. Brain Injury, 25(2), 169-178. doi:10.3109/02699052.2010.541894

Thabane, L., Ma, J., Chu, R., Cheng, J., Ismaila, A., Rios, L. P., . . Goldsmith, C. H. (2010). A tutorial on pilot studies: The what, why and how. BMC Med Res Methodol, 10, 1. doi:10.1186/1471-2288-10-1 
Thaler, N. S., Scott, J. G., Duff, K., Mold, J., \& Adams, R. L. (2013). RBANS cluster profiles in a geriatric community-dwelling sample. Clin Neuropsychol, 27(5), 794-807. doi:10.1080/13854046.2013.783121

Thickpenny-Davis, K. L., \& Barker-Collo, S. L. (2007). Evaluation of a structured group format memory rehabilitation program for adults following brain injury. J Head Trauma Rehabil, 22(5), 303-313. doi:10.1097/01.HTR.0000290975.09496.93

Tsaousides, T., D’Antonio, E., Varbanova, V., \& Spielman, L. (2014). Delivering group treatment via videoconference to inividuals with traumatic brain injury: A feasibility study. Neuropsychological Rehabilitation, 24(5), 784-803.

Verhaeghen, P., Marcoen, A., \& Goossens, L. (1992). Improving memory performance in the aged through mnemonic training: A meta-analytic study. Psychology and Aging, 7(2), 242-251. doi:10.1037/0882-7974.7.2.242

Wechsler, D. (2001). Wechsler Test of Adult Reading (WTAR). San Antonio, TX: Psychological Corporation.

Wechsler, D. (2009). Test of Premorbid Functioning (TOPF). San Antonio, TX: Psychological Corporation.

Whitehead, A. L., Julious, S. A., Cooper, C. L., \& Campbell, M. J. (2016). Estimating the sample size for a pilot randomised trial to minimise the overall trial sample size for the external pilot and main trial for a continuous outcome variable. Stat Methods Med Res, 25(3), 1057-1073. doi:10.1177/0962280215588241

Wilson, B. A. (1995). Management and remediation of memory problems in brain injured adults. In A. D. Baddeley, B. A. Wilson, \& F. N. Watts (Eds.), Handbook of memory disorders (pp. 683-710). Chichester, West Sussex, England: John Wiley \& Sons. 
Wilson, B. A., Clare, L., Baddeley, A. D., Cockburn, J., Watson, P., Tate, R., . . Crawford, J. R. (2008). Rivermead Behavioural Memory Test-Third Edition (RBMT-3) London, UK: Pearson Education Ltd.

Wilson, B. A., \& Moffatt, N. (1992). The development of group memory therapy. In N. M. B. A. Wilson (Ed.), The clinical management of memory problems (pp. 240-270). San Diego, CA: Singular Publishing Group.

Wise, E. A. (2004). Methods for analyzing psychotherapy outcomes: A review of clinical significance, reliable change, and recommendations for future directions. J Pers Assess, 82(1), 50-59. doi:10.1207/s15327752jpa8201_10

Yochim, B. P., Mueller, A. E., \& Segal, D. L. (2013). Late life anxiety is associated with decreased memory and executive functioning in community dwelling older adults. $J$ Anxiety Disord, 27(6), 567-575. doi:10.1016/j.janxdis.2012.10.010

Zigmond, A. S., \& Snaith, R. P. (1983). The Hospital Anxiety and Depression Scale. Acta Psychiatr Scand, 67(6), 361-370. 


\section{Appendix A}

\section{Group Psychoeducation and Questionnaire Script}

We are so grateful and excited to have each of you in our research study. Meeting in a group is a unique opportunity. The research suggests that people receive the same benefits from training offered in both groups or individually. In fact, research indicates that those who had memory training in a group do better on memory tests than those who had memory training one on one with a teacher. Groups offer advantages like increasing social support and talking with others can help us feel more confident and less anxious. Do you have any questions about meeting in a group? (listen and normalize fear). Thank you so much for sharing. Please feel free now and in the future to discuss concerns that come up.

I also wanted to take some time to explain a few questionnaires you'll be receiving. After every session we will be asking you to fill out two questionnaires. The first one asks about feedback you have regarding the program. The feedback includes eight statements regarding the program (read each statement aloud). You will be asked how much you agree or disagree with each of the eight statements. Answer options include strongly agree, somewhat agree, do not agree or disagree, somewhat disagree, or strongly disagree. Please choose one of those options for each question. Right below the multiple-choice options for each question is a space provided for you to leave comments about that statement. We are happy and excited to receive any feedback you have regarding the program. Please answer as honestly as you can and provide any specific feedback that comes to mind. There is no right or wrong answer; we only want to know your opinion. What questions do you have?

The second questionnaire asks about how you felt about the group. We know from research that those who feel engaged with their group have the best outcomes so we'll check in on this at the end of each session. We will ask you to rate the following 5 statements (read each statement aloud). You can rate the statement from 0 to 6 , in which 0 means not at all, 1 a little bit, 2 somewhat, 3 moderately, 4 quite a bit, 5 a great deal, or 6 extremely. For each statement please mark only one answer. Also, please think of the whole group during the most recent session when answering the question, not the group at other times. Again, we are most interested in your honest feedback and there aren't any right or wrong answers. What questions do you have? Please feel free to ask questions as you fill them out today and in the future. 


\section{Appendix B}

\section{Participant Feedback Form}

1. The training was useful?
a. Strongly agree
b. Somewhat agree
c. Do not agree or disagree
d. Somewhat disagree
e. Strongly disagree

Comment:

2. The educational portion was clear.
a. Strongly agree
b. Somewhat agree
c. Do not agree or disagree
d. Somewhat disagree
e. Strongly disagree

Comment:

3. I found the presentation helpful.
a. Strongly agree
b. Somewhat agree
c. Do not agree or disagree
d. Somewhat disagree
e. Strongly disagree

Comment:

4. The in-group practices were helpful.
a. Strongly agree
b. Somewhat agree
c. Do not agree or disagree
d. Somewhat disagree
e. Strongly disagree

Comment:

5. The therapist was clear and easy to follow.
a. Strongly agree
b. Somewhat agree
c. Do not agree or disagree
d. Somewhat disagree 
Comment:
e. Strongly disagree

6. The homework was useful.
a. Strongly agree
b. Somewhat agree
c. Do not agree or disagree
d. Somewhat disagree
e. Strongly disagree

Comment:

7. I did not encounter barriers to completing the homework.
a. Strongly agree
b. Somewhat agree
c. Do not agree or disagree
d. Somewhat disagree
e. Strongly disagree

Comment:

8. The training helped me in everyday life.
a. Strongly agree
b. Somewhat agree
c. Do not agree or disagree
d. Somewhat disagree
e. Strongly disagree

Comment:

Please leave comments below on the intervention, whether it was a positive or negative experience. 


\section{Appendix C}

FW: Kayla Dissertation Update/Question for Committee

Inbox

Michael Larson<michael_larson@byu.edu>

Mar 19, 2020, 4:19

to me

FYI from Dr. Cobia (see below). So, we are all cleared to be done with data collection. Thanks for your efforts.

Best,

Michael

Yep, I am fine with the adjustment as well.

-Derin

On Mar 19, 2020, at 10:20 AM, Mona Hopkins < Ramona_Hopkins@byu.edu> wrote:

I am good as well

Mona

From: Gary Burlingame < gary burlingame@byu.edu>

Sent: Thursday, March 19, 2020 10:20 AM 


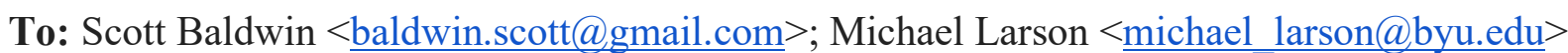

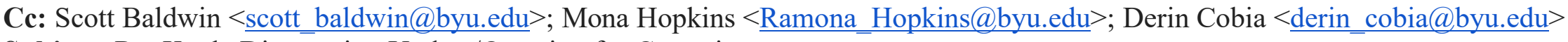
Subject: Re: Kayla Dissertation Update/Question for Committee

Me too

From: Scott Baldwin <baldwin.scott@gmail.com>

Date: Thursday, March 19, 2020 at 10:18 AM

To: Michael Larson $<$ michael_larson@byu.edu $>$

Cc: Scott Baldwin < scott_baldwin@byu.edu>, Gary Burlingame < gary_burlingame@byu.edu>, Ramona Hopkins

$<$ Ramona_Hopkins@byu.edu $>$, Derin Cobia $<$ derin_cobia@byu.edu $>$

Subject: Re: Kayla Dissertation Update/Question for Committee

I'm good with this given the circumstances.

Best,

Scott

On Thu, Mar 19, 2020 at 10:15 AM Michael Larson<michael_larson@byu.edu> wrote:

Hi all,

You are receiving this email because you are a member of Kayla Frodsham's dissertation committee. I purposely did not include Kayla on this email. As you recall, Kayla is doing a group-based memory intervention feasibility study in younger adults (portion completed) and older adults (this is why I am emailing). Kayla has been working very hard at her data collection. She had three groups going when the Covid-19 news hit and, because these are older adults in groups, we've had to shut down her data collection. Attached is an update on where her data collection is right now. As you can see, she has worked hard at this but shutting down two groups halfway through treatment and cancelling another group outright is going to cause difficulty. In short, she has run five young adult groups all the way through treatment and three older-adult groups, but the last two sessions for groups 2 and 3 were interrupted due to Covid-19 (they did not hold the last two sessions for group 2 and group 3, and many didn't come to the $5^{\text {th }}$ session once the alert went out about the virus). Several were willing to come in for the post-test evaluation, though, since it was just with one person instead of with a group. She has 39 pre-tests and 30 post-tests for younger adults and 26 pre-tests and 21 post-tests for older adults. Her data collection goals from her prospectus were to have 24 useable participants for both groups ( 48 useable total). Given the number that 
have problematic treatment data due to COVID, the unknown of when she will be able to run other groups, the fact that her whole undergrad team is graduating in April (so she will have to train a new team for the last older adult group or groups), and the cost of getting new manuals, testing supplies, etc. for multiple groups, and (finally) that this is a feasibility study, not an effectiveness study (and would have no chance of being published due to the discontinuation of the older adult groups), I propose that we adjust her dissertation to allow her to be completed with data collection with these numbers. She will still have an interesting feasibility story to tell and it will not take a lot of extra time/resources and stress on her to essentially re-do the older adult groups. Sorry for the long email, just wanted to explain. How do you, as committee members, feel about this?

Thanks for your input.

Sincerely,

Michael 